\title{
AVOIDANCE AND OVERGENERAL MEMORY
}

\author{
BY
}

\section{TIMOTHY JOHNSTONE GANLY}

\author{
A thesis \\ submitted to Victoria University of Wellington \\ in fulfilment of the requirements for the degree of \\ Doctor of Philosophy
}

Victoria University of Wellington

2018 


\begin{abstract}
Overgeneral memory is a phenomenon that occurs in depression in which people tend to remember temporally non-specific autobiographical memories. Overgeneral memory may be functional; by avoiding specific memories, potentially distressing emotions can avoided. This "functional avoidance" may be part of a repertoire of avoidance strategies people use when they are under stress. The question of the relationship between avoidance, stress, and overgeneral memory has been investigated using only laboratory-based stressors, and no previous research has examined the relationships in both non-clinical and clinical samples. Across four studies, this thesis investigated the relationships between avoidance and overgeneral memory in clinical and non-clinical samples and whether every-day stress moderates this relationship.

Studies 1, 2, and 4 engaged undergraduate samples in which mean depression scores were low (non-clinical samples). Study 3 engaged a sample from a university counselling service in which the mean depression score was high (clinical sample). Participants completed selfreport measures of avoidance and stress. They were also asked to remember specific events to a series of emotion cue words on the Autobiographical Memory Test (AMT). This thesis also investigated the possibility that avoidance may be associated with a reduction in memory performance on other tests of autobiographical memory besides the AMT, perhaps because other types of memories, not just specific, can be distressing. Thus, in Study 1, participants also completed the Autobiographical Memory Test-Reversed (AMT-R) in which they were asked to retrieve general memories. In addition, across studies, the pleasantness of events remembered to positive and negative cues was examined. In Study 4, the possible moderating role of rating pleasantness on the relationship between avoidance and overgeneral memory was examined.

Results from the non-clinical samples indicated higher avoidance was associated with less overgeneral remembering on the AMT. In the clinical sample, there were no significant relationships between avoidance and overgeneral memory. There were no significant relationships between avoidance and AMT-R performance. Overall, stress did not moderate the relationship between avoidance and overgeneral memory. Mean pleasantness ratings for events remembered to positive and negative cues were congruent with cue valence. However, individual positive and negative cues did not always elicit memories for pleasant and unpleasant events, respectively. Rating (vs. not rating) the pleasantness of remembered events did not moderate the relationship between avoidance and overgeneral memory. Overall, findings suggested that
\end{abstract}


functional avoidance is not part of a repertoire of avoidance strategies. Ironic process theory is discussed as an explanation for why higher avoidance was associated with a lower proportion of overgeneral memories in the non-clinical samples. 


\section{Acknowledgements}

I would like to thank my primary supervisor, Professor Karen Salmon for her unwavering support, encouragement, and kindness. I learned so much from her about carrying out psychological research, and her emphasis on the art of writing has been a great inspiration to me. I would also like to thank my second supervisor, Associate Professor John McDowall. His patience, invaluable advice, and sense of humour have meant a huge amount.

Thank you to the members of the Autobiographical Memory Lab over the years for their support. Learning with them has been great fun. Thank you especially to Carmen Campion for her help with data collection in Study 2. I'd like to give a special mention to Charlotte Gutenbunner and Lauren Bryce. I have worked alongside them over the past several years and in that time we have become great friends. They have been incredibly supportive and I could not have finished this thesis without them.

I am extremely grateful to Gerard Hoffman and his team at the Student Counselling Service at Victoria University of Wellington for all their help with Study 3. From our very first meeting when we discussed the possibility of recruiting participants through the counselling service, they were incredibly accommodating. Without them, Study 3 would never have been possible.

I have been very lucky to receive generous financial support from Victoria University in the form of the Victoria Doctoral Scholarship (2012-2015), Victoria Doctoral Submission Scholarship (2017), and FSRG travel grants (2014, 2015).

Thank you to Carol Fortune, Carrie Lynch, Liam Talbot, Lydia Rowden, Peter Ryan, Simon Mooney, and Sinead Connelly for being great friends throughout this process. They probably don't realise how much their support has meant to me. I have always known I could rely on them.

Finally, I would like to dedicate this thesis to my family. Despite being so far away, it has never felt that way. Thank you for everything. 


\section{Table of Contents}

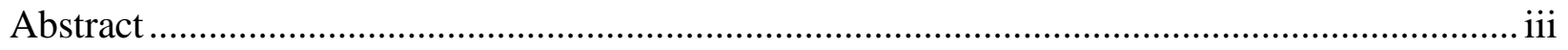

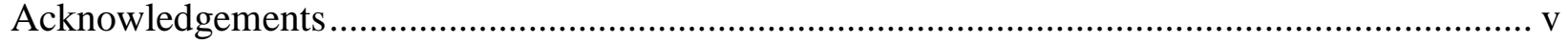

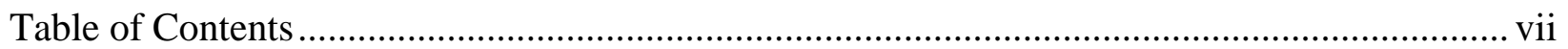

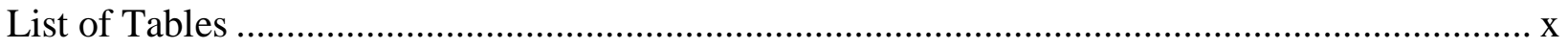

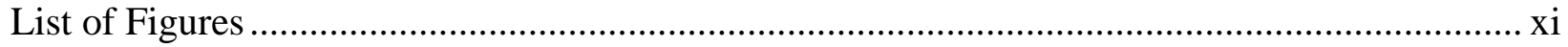

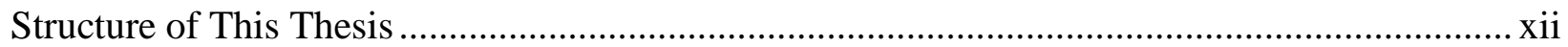

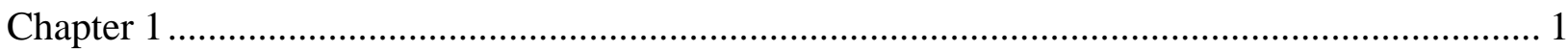

Overgeneral Memory, Avoidance, and Stress..................................................................... 1

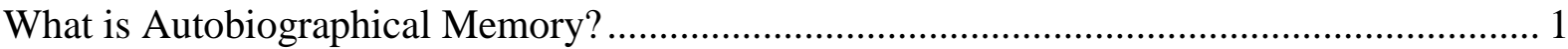

Temporal Specificity, Overgeneral Memory, and Psychopathology ........................................ 2

The Self-Memory System (SMS) ……………….................................................................. 4

The Self-Memory System account of overgeneral memory. ..................................................... 7

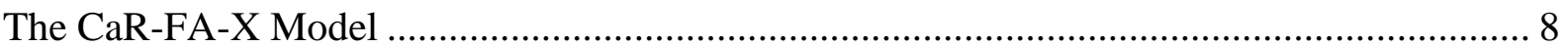

Measuring Overgeneral Memory: The Autobiographical Memory Test (AMT)..................... 10

Overgeneral Memory as an Avoidance Strategy in Psychologically Healthy People ............. 13

Evidence that Functional Avoidance is Context-Dependent: The Role of Stress.................... 18

Functional Avoidance in Clinical and Non-Clinical Groups .................................................... 22

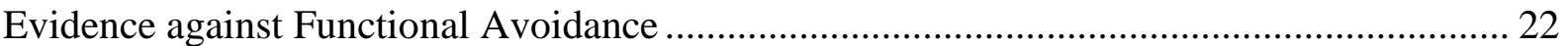

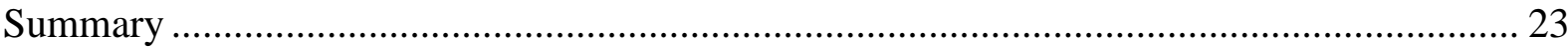

The Emotional Nature of Specific vs. General Memories ...................................................... 24

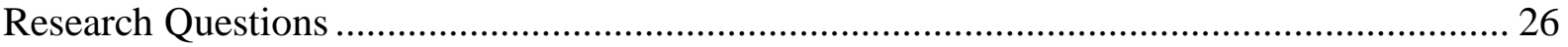

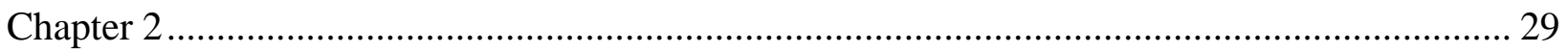

Study 1: The Relationship between Avoidance and Memory Specificity in a Non-clinical

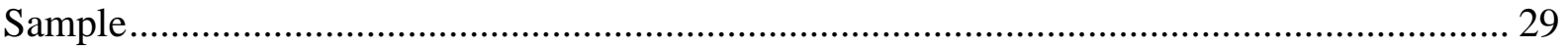

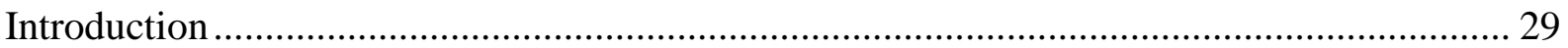

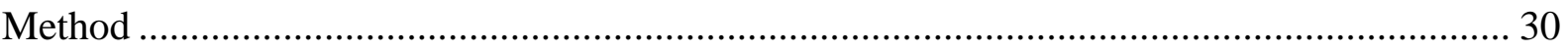

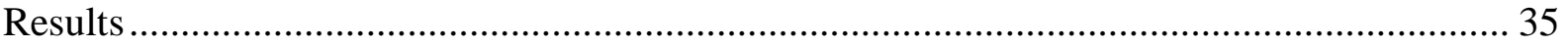

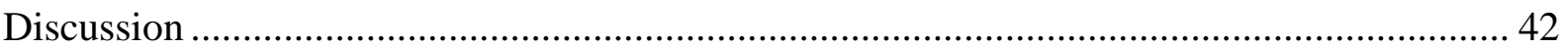

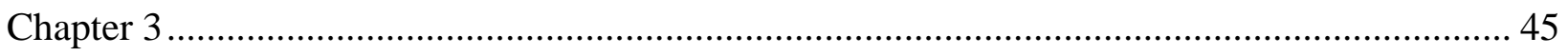

Study 2: Replication of AMT Findings from Study 1 ........................................................... 45 


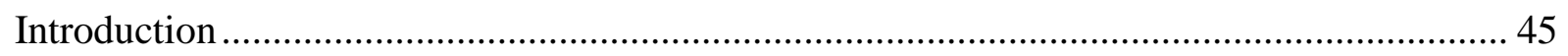

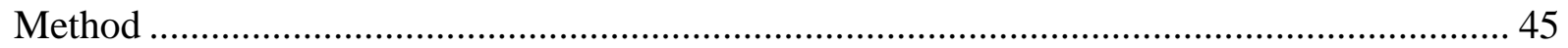

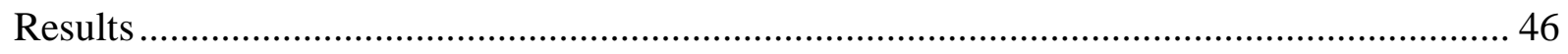

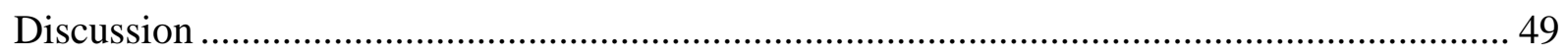

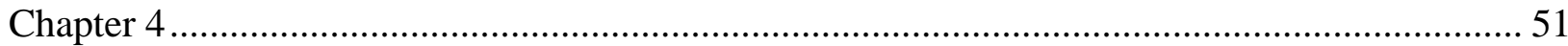

Study 3: The Relationships between Avoidance, Overgeneral Memory, and Stress in a

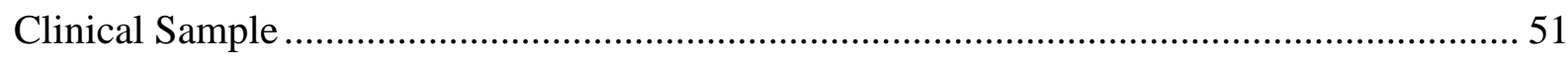

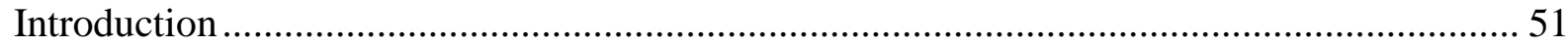

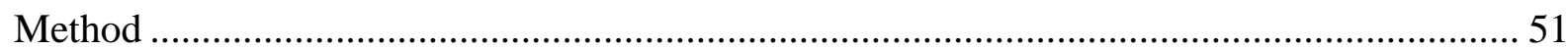

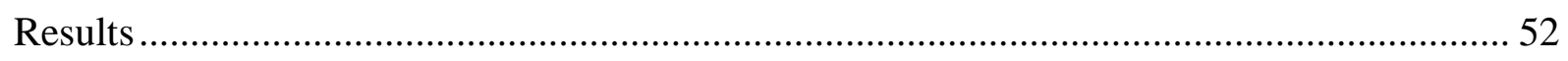

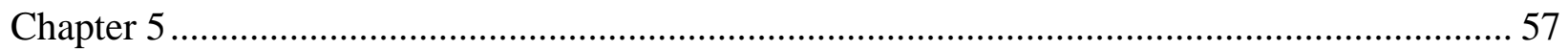

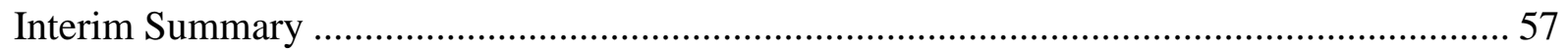

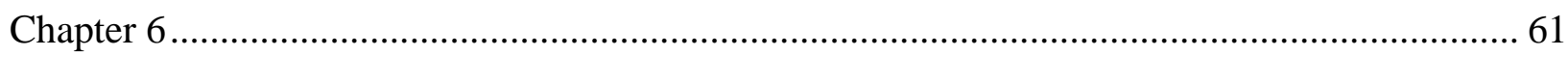

Study 4: The Influence of Rating Event Pleasantness on Overgeneral Memory ..................... 61

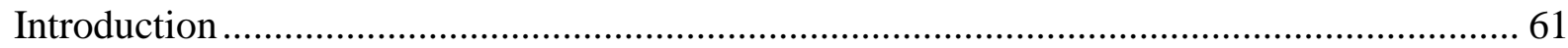

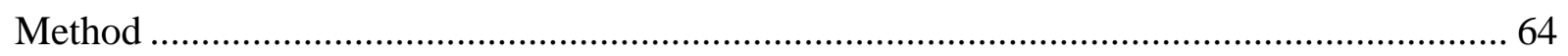

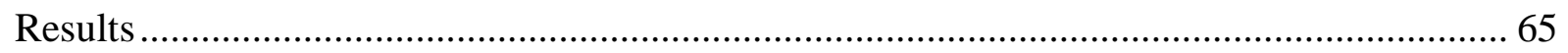

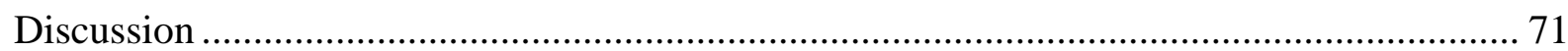

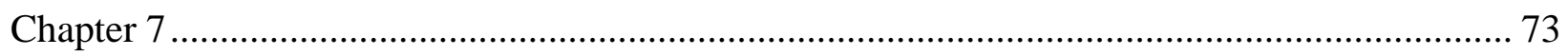

Research Synthesis: Meta-Analyses of the Relationships between Avoidance and AMT

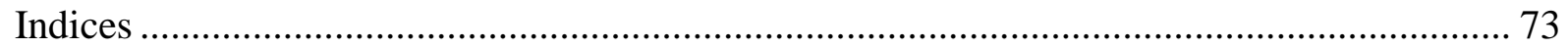

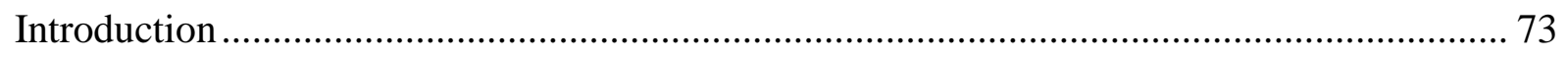

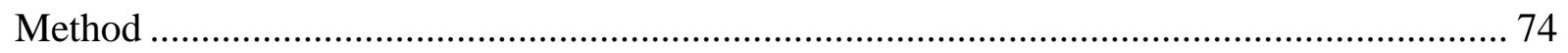

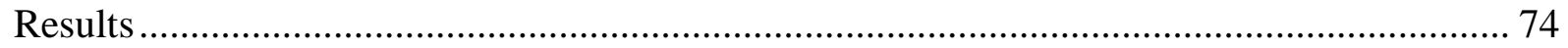

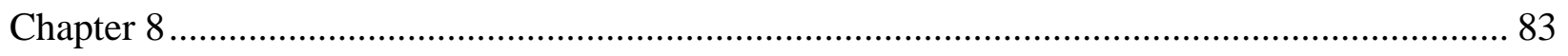

Pleasantness Ratings of Events Remembered to Positive and Negative Cues......................... 83

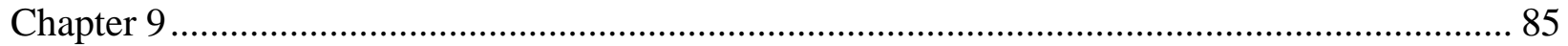

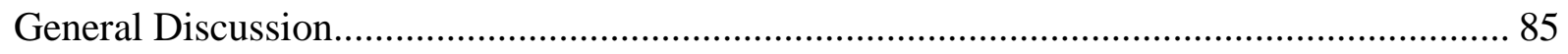

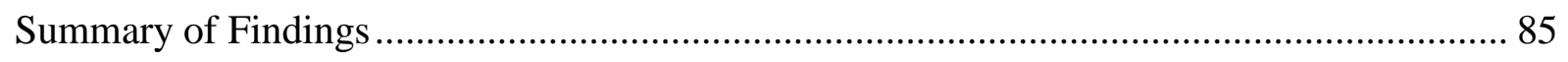

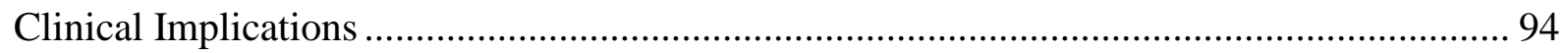

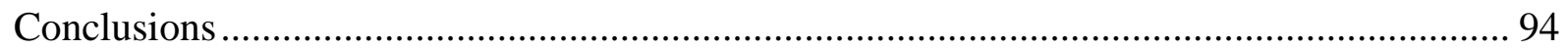




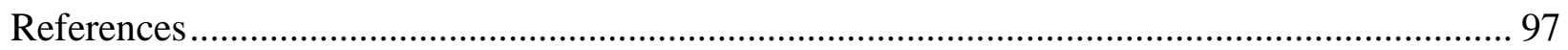

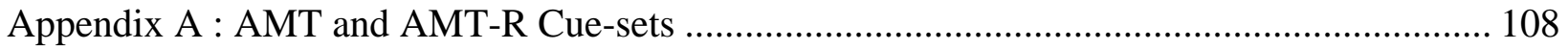

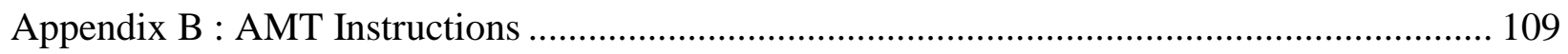

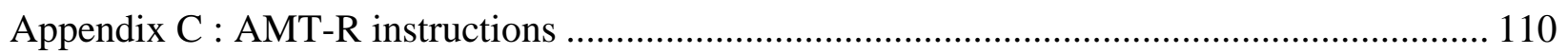

Appendix D : AMT and AMT-R Practice Trial Instructions.............................................. 111

Appendix E : Coding Scheme for AMT and AMT-R.................................................... 112

Appendix F : Self-Report Measures ............................................................................ 114 


\section{List of Tables}

Table 2.1 Descriptive Statistics for Study 1 Variables ................................................................ 37

Table 2.2 Correlations [and 95\% BCa Confidence Intervals] between Depression, Stress,

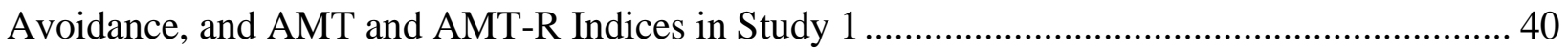

Table 3.1 Descriptive Statistics for Study 2 Variables ............................................................. 47

Table 3.2 Correlations [and 95\% BCa Confidence Intervals] between Depression, Stress,

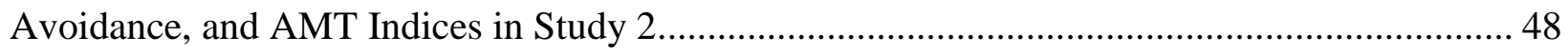

Table 4.1 Descriptive Statistics for Study 3 Variables ………………………………............ 53

Table 4.2 Correlations [and 95\% BCa Confidence Intervals] between Depression, Stress, Avoidance, and AMT Indices in Study 3 .................................................................................. 55

Table 6.1 Descriptive Statistics for Study 4 Variables in the Whole Sample and by Condition.. 66 Table 6.2 Correlations [and 95\% BCa Confidence Intervals] between Depression, Stress, Avoidance, and AMT Indices in Study 4 (NP and NPR Condition Combined).......................... 69

Table 7.1 Relationships between Proportion of Specific AMT Responses and Avoidance

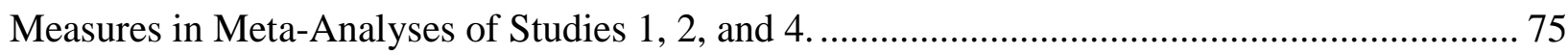

Table 7.2 Relationships between Proportion of Overgeneral AMT Responses and Avoidance

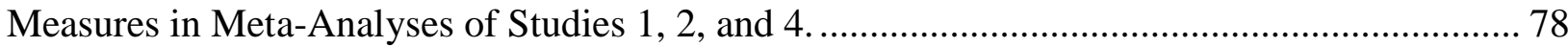

Table 8.1 Percent of Individual AMT Pleasantness Ratings to Positive and Negative Cues by

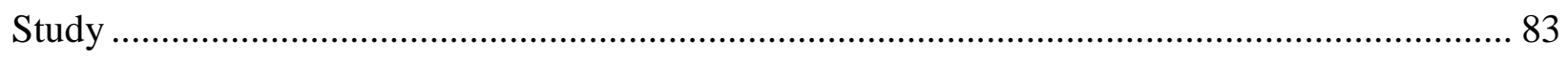

Table 8.2 Percent of Individual AMT-R Pleasantness Ratings to Positive and Negative Cues in Study 1 


\section{List of Figures}

Figure 6.1. Moderation by stress of the relationship between CBAS total score and proportion of

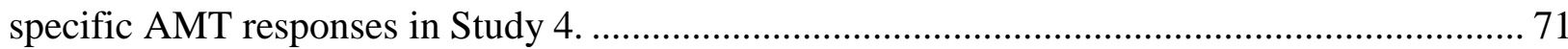

Figure 7.1. Forest Plots of Random-Effects (RE) Meta-Analyses Depicting Correlations (Pearson's $r$ s) between Avoidance Measures and AMT Proportion of Specific Memories. ........ 76 Figure 7.2. Forest Plots of Random-Effects (RE) Meta-Analyses Depicting Correlations (Pearson's $r$ s) between Avoidance Measures and AMT Proportion of Overgeneral Memories... 79 Figure 7.3. Relationships between avoidance measures and proportion of specific and overgeneral memories. 80 


\section{Structure of This Thesis}

Chapter 1 is a general introduction and provides the theoretical underpinnings to this program of research. It culminates in a summary of research aims. Chapters 2 to 4 describe studies 1 to 3, respectively. Chapter 5 is an interim summary of findings. Chapter 6 describes Study 4. Chapter 7 presents meta-analyses of findings from Studies 1, 2, and 4. Chapter 8 presents some further analyses of data from Studies 1 to 4 . Chapter 9 is a general discussion.

Some of the ideas and results reported in this thesis are presented in a published article that I was the first author for. It is:

Ganly, T. J., Salmon, K., \& McDowall, J. (2016). Is remembering less specifically part of an avoidant coping style? Associations between memory specificity, avoidant coping, and stress. Cognition and Emotion, 31(7).

doi:10.1080/02699931.2016.1227304

The article reports findings from Studies 1, 2, and 3 of the current thesis. Because of restrictions on space in the published article, it presented those findings in condensed format and abbreviated or omitted some research objectives. Thus, the current thesis can be considered an expansion of the published article. This thesis expands on the article by making reference to more recently published research, presenting some further analyses, and by describing a fourth study. There is some similar and identical wording used in this thesis to the published work. 


\section{Chapter 1}

\section{Overgeneral Memory, Avoidance, and Stress}

This thesis reports work that investigated the relationships between overgeneral memory, avoidance, and stress in three non-clinical samples and one clinical sample. In this chapter, I discusses the relevant existing literature and develop a series of research questions. Chapters 2 to 8 describe original empirical research that addresses those questions. Chapter 9 is a general discussion of the findings.

\section{What is Autobiographical Memory?}

According to Bernsten and Rubin (2012), "autobiographical memory is the ability to consciously remember personal events" (p. 333). Autobiographical memories are "transitory dynamic mental constructions" (Conway \& Pleydell-Pearce, 2000, p. 261), that is, they are created in consciousness, assembled from fragments of encoded representations of events and more abstract autobiographical knowledge as part of ongoing cognitive activity (Conway \& Jobson, 2012).

The notion that autobiographical memory is merely a system for constructing recollections belies its importance, however, and many authors have described it as vital to many aspects of human cognition. Conway and Pleydell-Pearce (2000) described autobiographical memory as the basis "to the self, for emotions, and for the experience of personhood" (p. 261), and its importance may be to maintain a stable sense of self across time (Conway, Singer, \& Tagini, 2004). Autobiographical memory is also conceptualised as being directive, that is, its purpose is to guide behaviour as we navigate our social world (Bernsten \& Rubin, 2012; Bluck, 2003; Bluck, Alea, Habermas, \& Rubin, 2005)

With respect to guiding behaviour, autobiographical memory is important for problem solving (Williams, 1996) and imagining possible future events (D'Argembeau, 2012). Indeed, in Klein's (2013) view, the importance of autobiographical memory lies in its role in futureoriented thinking; that is, autobiographical memory is part of an evolved system that helps us to anticipate and plan, to imagine the future effectively so that goals vital to survival can be pursued. Given the importance ascribed to autobiographical memory in its numerous accounts, it is no surprise that disruptions in its functioning are associated with negative psychological outcomes. 


\section{Temporal Specificity, Overgeneral Memory, and Psychopathology}

The relationship between autobiographical remembering and psychological wellbeing has been the subject of extensive previous research (e.g., Williams et al., 2007). One such line of research has dealt with the relationship between the temporal specificity of autobiographical memory and psychopathology. The temporal specificity of an autobiographical memory refers to whether it is a memory for a unique event or a category of events, and if unique, how long the event lasted. For example, a person asked to remember something that the word happy reminds them of might respond: "Two Saturdays ago, playing volleyball with my friend at the beach." Another person might respond: "We used to play volleyball at the beach". The first memory is temporally specific, referring to a specific, unique event that happened over a relatively short period of time (less than one day). The second memory is temporally non-specific; rather than referring to a specific, unique event, it refers to a category of related events, the times spent playing volleyball at the beach. In this way, it is known as a "categoric" memory. A memory such as "On holiday last year in France" is known as an "extended memory" because it is, although unique, also temporally non-specific, referring to a relatively extended period of time. Due to their temporal non-specificity, categoric and extended memories are referred to as general memories (Moore \& Zoellner, 2007; Williams et al., 2007).

Some people, notably, those who are depressed, when asked to retrieve a specific memory, are more likely to experience a difficulty with this compared with a person who is not depressed (Williams et al., 2007). In particular, rather than responding with a specific memory, they respond with a general memory instead. When a person has this tendency to remember general rather than specific memories, they are said to be exhibiting overgeneral memory (Williams, 1996).

The link between overgeneral memory and depression has spurred research interest over the past 30 years (Sumner, 2012; Williams et al., 2007). People who are depressed exhibit higher levels of overgeneral memory than those who are not depressed (van Vreeswijk \& de Wilde, 2004). For example, when compared with non-depressed controls, depressed individuals report more general memories (e.g., Kuyken \& Dalgleish, 1995), and fewer specific memories (e.g., Goddard, Dritschel, \& Burton, 1996). Overgeneral memory is also a predictor of onset of depression (Sumner, Griffith, \& Mineka, 2011) and a worse course of depression (Sumner, Griffith, \& Mineka, 2010). In addition to its association with depression, overgeneral memory is 
also associated with posttraumatic stress disorder (PTSD; Moore \& Zoellner, 2007; Sumner, 2012; Williams et al., 2007). For example, overgeneral memory predicts onset of PTSD after trauma, and overgeneral memory is found in people with PTSD even when controlling for comorbid depression (Moore \& Zoellner, 2007).

Studies that found an association between trauma and overgeneral memory lead to affectregulation accounts of overgeneral memory (Williams, 1996; Williams, Stiles, \& Shapiro, 1999). These theories proposed that overgeneral memory is the result of attempting to avoid traumatic specific memories. In short, by avoiding the particulars of specific traumatic events, their accompanying negative emotions can also be avoided. Thus, from this perspective, overgeneral memory is an attempt at emotion regulation in the face of traumatic memories. Recent reviews of the literature have concluded, however, that trauma alone is unlikely to be the primary mechanism that leads to overgeneral memory (Moore \& Zoellner, 2007; Sumner, Mineka, \& McAdams, 2012).

Two points are worth noting here. First, in order to extend earlier-trauma based theories of overgeneral memory, research conducted more recently has investigated the emotionregulation account of overgeneral memory in psychologically healthy people (e.g., Debeer, Raes, Williams, \& Hermans, 2011; Hermans, Defranc, Raes, Williams, \& Eelen, 2005; Raes, Hermans, de Decker, Eelen, \& Williams, 2003). A recent version of the emotion-regulation account, "functional avoidance" (Williams et al., 2007), discussed in detail later in this thesis, is primarily a description of the link between psychopathology and overgeneral memory as an avoidance process. However, it has been expanded in recent research to include an explanation of the contexts in which psychologically healthy people use overgeneral memory in an adaptive way to ward off negative emotions (Debeer et al., 2012, 2011; Debeer, Raes, Williams, \& Hermans, 2013). The research in this area is minimal however, with unanswered questions and some contradictory findings.

Second, there has been a recent renewed interest in ideas put forth by Ferster (1973) that avoidance processes are a key component of depression, not only trauma- and anxiety-related psychopathology (Moulds \& Kandris, 2007; Moulds, Kandris, Starr, \& Wong, 2007; Ottenbreit \& Dobson, 2004; Quigley, Wen, \& Dobson, 2017; Trew, 2011). Given these two points, there is a need for investigation of overgeneral memory as an emotion-regulating avoidance process in both psychologically healthy people and depressed people. The current thesis is a response to 
this need.

\section{The Self-Memory System (SMS)}

In this section, I describe the Self-Memory System (SMS; Conway, 2005; Conway \& Pleydell-Pearce, 2000; Conway et al., 2004), a conceptual framework that describes the structure and functioning of autobiographical memory. In addition, I also present two other ideas of importance to this thesis that Conway \& Pleydell-Pearce (2000) contributed, a description of the relationship between autobiographical memory and emotion, and a description of how overgeneral memory can be explained within the SMS framework. The purpose of doing this is to provide a theoretical backdrop so that pursuant theories such as Williams et al.'s (2007) CaRFA-X model of overgeneral memory can be understood, and to lay the foundation for addressing this thesis' main research questions.

Overview. In creating the SMS, its authors postulated the autobiographical memory knowledge base, the working self, and the conceptual self. The knowledge base is a highlyconnected, hierarchical, associative network of event-representations and abstract knowledge. The working self is comprised of a person's currently active goals. It interacts with the knowledge base and is overseen by a set of control processes. In conjunction with the conceptual self, which contains abstractions such as attitudes and beliefs, the working self guides the encoding, consolidation, and retrieval of memories. Thus, in describing the SMS, the authors attempted to integrate the ideas of autobiographical memory and self into a coherent conceptual framework.

Autobiographical memory knowledge base. In the SMS, the autobiographical knowledge base is conceptualised as a hierarchy with several levels ranging from less specific, abstract representations of events at the "top" of the hierarchy to more specific, concrete representations at the "bottom". At the top of the hierarchy, representations are of lifetime periods, e.g., "My time at university". At the intermediary level, representations are of general events, that is, summaries of multiple events, e.g., "Weekends at the beach"; or extended events, e.g., "my holiday in France". At the bottom of the hierarchy, representations are of unique experiences, event-specific knowledge (ESK), that are rich in sensory-perceptual details. The knowledge base is nested: Representations at each level of the hierarchy can index or cue representations at neighbouring levels. The knowledge base is in a continual state of flux with representations constantly being activated by internal and external cues. This necessitates the 
involvement of inhibitory processes that prevent representations from spontaneously emerging into consciousness and disrupting the currently active goals of the working self. Within the SMS, a consciously recalled memory occurs when a pattern of activation stabilises within the knowledge base and is connected to the goals of the working self. There are two ways that this can occur, described below.

\section{Types of remembering in the SMS: Generative and direct retrieval. Research into} autobiographical memory necessarily requires participants to retrieve autobiographical memories. For that reason, it is important to understand how the retrieval of autobiographical memories operates. Therefore, I now describe the two types of memory retrieval said to occur within the SMS, generative retrieval and direct retrieval.

Generative retrieval is the effortful search of the autobiographical knowledge base that occurs when, for example, a person purposefully attempts to retrieve a specific memory. The process consists of three phases, the first two of which may occur simultaneously. In the first phase, the elaboration phase, a mnemonic cue is elaborated by verbal association, that is, made relevant to the self. Williams et al. (1999) described this stage as generating a response to the question implied in the mnemonic cue. For example, if the cue is happy, it may be elaborated by answering the implied question: "What types of thing make me happy?" In the second phase, the retrieval model phase, criteria are established against which representations activated during search can be evaluated. These criteria are encapsulated within the working self's goals as a mental model known as the retrieval model. In effect, the retrieval model is a mental representation of the desired outcome for a particular retrieval search. For example, if a person is asked to remember a specific event that the word happy reminds them of, this activates a verbal associate of the cue that is relevant to the self (elaboration phase), e.g., "music", and establishes the search criteria that the event must be specific and be a reminder of the word happy (retrieval model phase).

The third phase of generative retrieval is the search phase. The most common entry point into the autobiographical knowledge base for beginning the search phase tends to be at the level of extended or general events. To continue the example from above, this might be the general event, "The times I played with the orchestra". Activation gradually spreads via the indexes of the knowledge base to representations at all levels of the hierarchy, including those at the level of ESK (e.g., "My first concert in which we received a standing ovation”). As retrieval search 
proceeds, control processes are involved in evaluating currently activated representations against the retrieval model's criteria, selecting those that are appropriate for further refinement, and inhibiting those that are inappropriate. The retrieval model shapes the emergence of the pattern of activation, with each activated representation potentially serving as a cue to be elaborated further so that the search can continue. Gradually, activated representations attach to the retrieval model, and retrieval search finishes when activated representations meet the established criteria specified in the retrieval model. Thus, generative retrieval is an iterative "search-elaborateevaluate retrieval cycle" (Conway \& Pleydell-Pearce, 2000, p. 268).

The second type of retrieval, direct retrieval, differs from generative retrieval in that the control processes of the working self are not involved. In contrast to the effortful, strategic construction of a memory that occurs during generative retrieval, direct retrieval occurs spontaneously and unexpectedly, corresponding to the subjective experience of a memory "popping into mind". Direct retrieval occurs when an internal or external cue directly activates representations at the level of ESK, and either (a) control processes select the activated representation and it enters awareness leading to the experience of unexpected conscious recall, or (b) inhibitory processes are overcome and the SMS is automatically put into retrieval mode as the activated representation is linked to the working self's currently activated goals, again leading to the experience of unexpected conscious recall. Importantly, and in contrast to generative retrieval, there is no elaboration or search phase involved in direct retrieval.

In summary, Conway and Pleydell-Pearce (2000) described remembering autobiographical memories as a dynamic constructive process that operates on a hierarchy of autobiographical knowledge. In creating this framework, they provided a theoretical basis for making distinctions between types of autobiographical memories (e.g., specific vs. general) in terms of their underlying knowledge structures and retrieval processes.

Emotions and goals in the Self-Memory System. With respect to explaining the phenomenon of overgeneral memory, the SMS makes another valuable contribution: a description of the relationship between autobiographical memory and emotion.

According to Conway \& Pleydell-Pearce (2000), the functioning of human memory is goal-oriented in nature, and this idea is encapsulated in the SMS's working self, a construct representing a person's currently active goals. In the SMS framework, emotion is conceptualised as a marker for goal-relevant experiences. Specifically, positive and negative emotions are the 
results of goal success and failure, respectively. In addition, experiences of success or failure at reaching goals are the essence of what is encoded in memory. Thus, there is a link between emotions and specific autobiographical memories; it is emotional, experiential outcomes that are encoded as specific autobiographical memories. In other words, emotional memories represent the outcomes of plans for goal attainment. Plans and goals themselves might not be encoded, or at least might not be encoded in such a way as to be available to consciousness.

Based on these ideas about the relationships between emotions and goals, Conway and Pleydell-Pearce (2000) proposed that remembering specific emotional material may be destabilising because it may disrupt currently active goal-processing. Accordingly, Conway and Pleydell-Pearce argued, the working self carefully orchestrates the arrival of autobiographical memories into consciousness such that the system is not cast into disarray as goal-relevant (emotional) material is re-instantiated. Human memory has evolved to manage this process carefully: Emotional and non-emotional aspects of autobiographical memories are carefully integrated during remembering, so re-experiencing associated emotions is, generally, not destabilising. The potentially destabilising nature of some autobiographical memories is the basis for Conway and Pleydell-Pearce's explanation for overgeneral memory.

\section{The Self-Memory System account of overgeneral memory.}

Conway and Pleydell-Pearce's (2000) account of overgeneral memory was based in part on Williams' (1996) account. In short, Williams proposed an emotion-regulation model of overgeneral memory, suggesting potentially aversive (specific) memories are avoided, leading to an "overelaboration" (p. 261) of nonspecific material. That is, attempts at avoiding specific memories result in non-specific, categoric representations being over-rehearsed, thus increasing the likelihood over time of being activated during subsequent retrieval search.

Conway and Pleydell-Pearce (2000) drew on Williams's (1996) description to lay the foundation for explaining overgeneral memory in terms of the SMS. From the perspective of the SMS framework, Conway and Pleydell-Pearce argued, remembering temporally non-specific memories is an adaptive response to potentially destabilising (emotional) material encountered during retrieval search. In order to protect the working self's currently active goals, retrieval search is terminated when potentially destabilising material is encountered. It is the specific aspects of memories, those at the "bottom" of the autobiographical knowledge base's hierarchy, ESK, that hold these emotional representations. Hence, retrieval searches that terminate in this 
way integrate only general representations into their retrieval models. It is therefore only general representations that enter consciousness and that can be reported. Conway and Pleydell-Pearce described overgeneral memory as a "dysfacilitation" (p. 282) of retrieval search: It is what occurs when a person prevents (dysfacilitates) the integration of specific representations into the retrieval model during remembering.

In summary, by describing the links between emotion, goals, and autobiographical memory retrieval, Conway and Pleydell-Pearce (2000) provided a theoretical grounding for understanding Williams' (1996) affect-regulation account of overgeneral memory, that it may occur, in part, because a person is motivated to avoid the unpleasant emotions that accompany some specific memories. These ideas were greatly elaborated in the most comprehensive explanation of overgeneral memory, the CaR-FA-X model (Williams et al., 2007), discussed in the next section.

\section{The CaR-FA-X Model}

The CaR-FA-X model (Williams et al., 2007) is an attempt to explain overgeneral memory and its link with psychopathology. It proposes three mechanisms, functional avoidance (FA), capture and rumination (CaR), and deficits in executive capacities (X) that, possibly in interaction, lead to negative outcomes and psychopathology. In the following description of the model, I pay particular attention to the FA component.

Following Williams' (1996) affect-regulation account of overgeneral memory and Conway and Pleydell-Pearce’s (2000) “dysfacilitation” account, Williams et al. (2007) proposed that a key causal mechanism in the development of overgeneral memory is "functional avoidance" (FA). FA refers to a person's attempts to keep at bay the negative emotions that might accompany specific memories.

According to the CaR-FA-X model, FA has three main features. First, avoiding specific memories to regulate emotion is used flexibly by psychologically healthy people; that is, they modulate the specificity of their remembering as needed. In other words, a person might exhibit overgeneral memory only when they encounter potentially destabilising negative emotions associated with some specific memories. In this way, FA is not maladaptive in of itself. Rather, because encountering emotional material during retrieval search may be potentially destabilising to the working self (Conway \& Pleydell-Pearce, 2000), functional avoidance is adaptive, a necessary part of remembering. Second, for some people (perhaps those motivated to avoid some 
specific memories because the memories are extremely aversive), FA must not only be applied when unpleasant events are remembered, but when remembering memories of all types. This is because the autobiographical memory knowledge base is a densely connected network of associated knowledge that is constantly in a state of flux, and, as Williams et al. (2007) pointed out, it is somewhat unpredictable. In short, the possibility of activating aversive memory representations is always present, and for FA to be successful, therefore, it needs to be applied to all remembering. Third, FA may be shaped by negative reinforcement as painful emotions that come with specific memories are repeatedly avoided. That is, over time, the emotion-regulation strategy of avoiding specific memories increases in frequency because it takes away the negative emotions associated with some specific memories. Williams et al. argued that over time, the FA strategy can develop from a flexible to an inflexible style of remembering.

The CaR-FA-X model also suggests that due to decreased flexibility, a person may recall general memories more often, leading to their over-rehearsal, and greatly increasing their future accessibility to consciousness and likelihood of their activation during subsequent retrieval. When these over-rehearsed, general representations are encountered during search, attention may be diverted or "captured" by abstract, self-relevant, ruminative themes present within. This Capture and Rumination $(\mathrm{CaR})$ phenomenon impedes the progression of retrieval to the level of specific representations. In combination with any deficits in executive capacities $(\mathrm{X})$ that guide retrieval, these processes are said to result in overgeneral memory.

The CaR-FA-X model links overgeneral memory to psychopathology in two ways. First, overgeneral memory's underlying processes, described above, can themselves be vulnerabilities to psychopathology. For example, the tendency to start ruminating during retrieval search (capture and rumination) is detrimental because rumination itself is depressogenic (NolenHoeksema, Wisco, \& Lyubomirsky, 2008). Second, the underlying processes directly, or through their effect on autobiographical memory, lead to secondary deficits such as impaired problem solving and difficulty imagining future events, which themselves contribute to psychopathology (Williams et al., 2007).

In summary, the CaR-FA-X model proposes three underlying mechanisms which individually, and through their effect on overgeneral memory, contribute to psychopathology. The most relevant of the mechanisms to this thesis is functional avoidance, discussed later in this chapter. 


\section{Measuring Overgeneral Memory: The Autobiographical Memory Test (AMT)}

Many of the studies reviewed in the current thesis have made use of a test of overgeneral memory called the Autobiographical Memory Test (Williams \& Broadbent, 1986), a task in which participants are asked to retrieve an autobiographical memory to each of a series of cue words. One of the goals of the current thesis was to replicate findings of previous studies that have used the AMT. Accordingly, in this section, I give an overview of the AMT to aid in understanding previous research described in this chapter and to provide a context for the methodology used in the current thesis.

General description. The AMT has several variants (Griffith et al., 2012). "AMT” often refers to a family of similar tasks that differ in their administration in terms of, for example, number of trials, cue words, and mode of responding (written, spoken). In this section, I provide a general description of the AMT, highlighting methodological details that tend to vary between different studies.

The AMT is administered individually or in groups. First, participants are given instructions that they should remember an autobiographical memory that each of a series of cue words reminds them of. In the "traditional instructions" version of the AMT (Williams \& Broadbent, 1986), participants are asked to respond with a specific memory for each cue word, and they are given examples of what a specific memory is. In the "minimal instructions" version (Mi-AMT; Debeer, Hermans, \& Raes, 2009), the instruction to provide a specific response is omitted. Participants are also told that each response should be unique, that is, the same response should not be provided to different cues. Typically, practice cues are also administered and examples of appropriate and inappropriate responses are given.

Each trial of the AMT consists of presenting the cue word to the participant by reading it aloud and/or presenting it visually (e.g., on a printed card, computer screen, or printed page of a response-booklet). The participant is given a fixed amount of time (usually 30 or 60 seconds) to respond. In the traditional instructions version, if the participant does not respond with a specific memory within that time, the test moves on to the next trial. If the participant responds within the time allowed but the response is not specific (e.g., a categoric memory), the participant is asked if they can think of a specific event related to the cue. This prompt is omitted if administration is in a group setting. Thus, when administered individually, first responses that are not specific lead to a prompt from the researcher that serves as a reminder of the instruction to be specific. 
Irrespective of subsequent responses within each trial, however, it is only the first response given by the participant that is coded and included in the final specificity and overgenerality scores. In the Mi-AMT, any response is accepted on each trial and no prompting occurs. Additional instructions may also include that the respondent should only respond with memories that are older than a week (e.g., Hermans et al., 2005). This is to safeguard against potential recency effects where memories for events that happened recently are more easily brought to mind.

As mentioned above, each AMT trial involves presenting the participant with a cue word that will serve as a starting point for their memory retrieval. Cues typically consist of positive (e.g., "happy") and negative (e.g., "sad") emotion words. Neutral valence (e.g., "door”) words are sometimes also used. The number of cues administered can vary, though five positive and five negative cues are typically administered.

Coding AMT responses. Each AMT response is coded as one of several possible response types. Although there is some variation amongst studies (see Griffith et al., 2012), typically, each response is coded as specific, categoric, extended, semantic associate, error, or omission. The specific code is assigned to responses that describe a memory for a temporally specific event, that is, one that lasted less than one day and that happened at a particular time and place, e.g., "playing soccer on the beach two weekends ago". The categoric code is assigned to responses that describe a category of events, e.g., "the times we used to go to the beach". The extended code is assigned to responses that correspond to an event that lasted longer than one day, e.g., "when we went on holiday to France". The semantic associate code is assigned to responses best described as semantic associates of the queue rather than pertaining to an event per se, e.g., if in response to the keyword "happy", the response is "beach". The error code is assigned if the participant reports a non-memory such as a future-oriented thought, responds nonsensically, gives a response that is a repetition of a previous response, or responds with an event that happened less than one week ago. An omission is coded if no response is given. Coding serves the purpose of deriving the variables of interest that will be used in statistical analyses.

There is much variation between studies in what variable derived from the AMT is used in analyses. For example, Hermans et al. (2008) used the number of specific responses; Debeer et al. (2012) used (a) the proportion of specific responses excluding omissions and (b) the proportion of categoric responses excluding omissions; Kuyken, Howell, and Dalgleish (2006) 
used (a) the number of specific responses and (b) the number of overgeneral responses (categoric and extended responses combined). Some research presents AMT indices created by collapsing cues across valences (e.g., specificity to positive and negative cues combined), whereas some research presents AMT indices for cue valences separately (e.g., specificity to positive cues, specificity to negative cues); there is some debate in the literature over which is the best approach (Griffith et al., 2012).

AMT indices used in the current studies. The AMT indices used in the current studies were specificity (i.e., proportion of specific memories excluding omissions) and overgenerality (i.e, proportion of categoric and extended memories excluding omissions). I chose to examine specificity and overgenerality for two reasons. First, reductions in specificity and increases in overgenerality may not be measures of the same construct; healthy individuals may use reduced specificity flexibly, but without necessarily remembering in an overgeneral way (Raes, Hermans, Williams, \& Eelen, 2006), whereas people who are depressed my use it inflexibly, that is, they may habitually truncate retrieval at the level of overgeneral memories (Hermans et al., 2008; Williams et al., 2007). Second, some previous research has examined only one of these indices (e.g., Hermans et al.), potentially leading to an incomplete picture of the relationships between these variables. I excluded omission in the calculation of these indices because their meaning is ambiguous. An omission could indicate that no memory has been retrieved, that a memory has been retrieved but the participant has chosen not to report it, or that a memory has been retrieved but the participant recognises that it does not meet the task-demands (Crane, Barnhofer, \& Williams, 2007; Griffith et al., 2012).

Categoric and extended responses were included in the calculation of the index of overgeneral memory for two reasons. First, previous studies have conceptualised overgeneral memory this way (e.g., Bunnell \& Greenhoot, 2012, 2017; Kuyken et al., 2006). Second, based on Conway and Pleydell-Pearce's (2000) description of the generative retrieval of overgeneral memories (discussed above), the presence of extended or categoric representations (but the absence of specific representations) in a response are an indication that retrieval search has occurred but that specific representations were not activated. According to Conway and PleydellPearce, the third phase (search phase) of generative retrieval begins with the activation of an extended or categoric representation within the autobiographical memory knowledge base. Thus, either of these two types of representation may be present in a temporally non-specific report. In 
other words, both are "signatures" of the initialisation of the search phase of generative retrieval. Therefore, if a response includes categoric or extended components but does not include specific components, this indicates that retrieval has proceeded beyond the first two phases (elaboration and retrieval-model phases) to the search phase but failed to activate representations at the level of event-specific knowledge (ESK). In short, categoric and extended responses indicate the search phase has occurred, but has not accessed ESK: the essence of overgeneral memory.

The AMT has been shown to have a one-factor structure (Griffith et al., 2009; Heron et al., 2012). For this reason, I chose to collapse positive and negative AMT cues across valences when creating AMT indices.

\section{Overgeneral Memory as an Avoidance Strategy in Psychologically Healthy People}

The idea that remembering less specifically might be considered an avoidance strategy in psychologically healthy people has been tested in numerous ways, but broadly speaking, this literature can be organised into four main lines of research. First, studies have investigated whether remembering less specifically is associated with higher levels of self-reported avoidance behaviours and cognitions (Hermans et al., 2005). Second, studies have investigated whether people who remember less specifically experience less distress following a stressful situation, that is, that remembering less specifically is protective against the negative emotional consequences of a negative event (Raes et al., 2003, 2006). Third, studies have investigated whether memory specificity decreases when remembering itself is perceived as threatening but only for those people high on avoidant coping (Debeer et al., 2011). Fourth, studies have investigated whether memory specificity might decrease after a stressful situation but only for those people high on avoidant coping (Debeer et al., 2012). In the following sections, I first describe what is meant by avoidance, then describe the aforementioned lines of research in detail.

What is avoidance? According to Ottenbreit and Dobson (2004), avoidance is "refraining from, or escaping from, an action, person or thing" (p. 293). They argued that avoidance is a response to a problematic "external situation or task or an internal thought, emotion or experience" (p. 297). The term avoidance, has many different treatments in the psychological literature, and as Ottenbreit and Dobson state, this literature is heterogeneous; there are numerous domains in which the concept has been discussed. For example, some have viewed avoidance as a counterpart to approach, a basic unit in motivational systems that govern 
how humans and non-human animals determine courses of action (e.g., Gray \& McNaughton, 2000) or resolve discrepancies between desired and actual states (e.g., Carver, 2006; Carver \& Scheier, 1990; E. T. Higgins, 1987, 1997). Avoidance has also been discussed as a strategy that people may use to cope with stressful life situations (Moos \& Schaefer, 1993), an ineffective problem-solving style (D’Zurilla \& Nezu, 1999), as a transdiagnostic marker of psychopathology (Hayes, Strosahl, \& Wilson, 2012; Mansell, Harvey, Watkins, \& Shafran, 2009), or as a feature of specific disorders such as depression (Ferster, 1973) or anxiety (Borkovec \& Roemer, 1995).

I will restrict the present discussion to conceptualisations of avoidance investigated in the overgeneral memory literature most pertinent to the current thesis. Specifically, Hermans (2005), who investigated the possibility that reduced memory specificity was part of a wider repertoire of avoidance strategies, used the Cognitive-Behavioural Avoidance Scale (CBAS; Ottenbreit \& Dobson, 2004), the Acceptance and Action Questionnaire (Hayes et al., 2004), and the White Bear Suppression Inventory (WBSI; Wegner \& Zanakos, 1994). I will now discuss each of these measures with a view to elucidating the avoidance construct as it most relevantly pertains to the current thesis.

The CBAS was created in order to better understand the relationship between avoidance and depression. Evaluation of a large initial item pool indicated that avoidance strategies could be divided into cognitive vs. behavioural and social vs. non-social dimensions (Ottenbreit \& Dobson, 2004). Based on moderate correlations between the resulting subscales (cognitive social, cognitive nonsocial, behavioural social, behavioural nonsocial), the authors described the scale as tapping "distinct, but related, constructs" (p. 303). In other words, people tend to vary in the types of avoidance strategies they use, but to some extent, they tend to use avoidance strategies across multiple domains.

The purpose of developing the AAQ was to capture the construct of experiential avoidance, defined by Hayes, Wilson, Gifford, Follette, \& Strosahl (1996) as "the phenomenon that occurs when a person is unwilling to remain in contact with particular private experiences (e.g., bodily sensations, emotions, thoughts, memories, behavioral predispositions) and takes steps to alter the form or frequency of these events and the contexts that occasion them" ( $p$. 1154). In creating the AAQ, Hayes et al. (2004) drew on relational frame theory (RFT; Hayes, Barnes-Holmes, \& Roche, 2001) that states that as people learn about and categorise aversive states using language (e.g., "anxiety is bad"), language itself takes on the ability to reinstate such 
aversive states. According to the theory, the ensuing "excessive entanglements with or fusion with thoughts" (Hayes et al., 1996, p. 1155), is the basis for much psychological suffering and the reason why a person may want to avoid internal experiences (Hayes et al., 2004, 2001, 2012, 1996).

The WBSI is a measure of a person's tendency to "chronically suppress thoughts" (Wegner \& Zanakos, 1994). The basis of the WBSI is the thought suppression literature in which the oft-used laboratory paradigm involves asking participants to deliberately not think a target thought. The effects of thought suppression (i.e., the occurrence or non-occurrence of the thought) are then examined. Foundational to this research domain is the finding that deliberate thought suppression can result in the increased frequency of the target thought, known as an enhancement effect (see Magee, Harden, \& Teachman, 2012 for a review). Wegner and Zanakos argued that engaging in thought suppression over long periods plays a central role in psychopathology, and they developed the WBSI to examine the psychology of people who engage in this activity.

In summary, the self-report measures of avoidant coping strategies administered by Hermans et al. (2005), the AAQ, CBAS, and WBSI, emerged from somewhat different research domains. However, their commonalities, as they are relevant to this thesis, are of note. Each conceptualisation encompasses the idea that a person may attempt to avoid some aspect of their own cognition (e.g., an emotion, thought, or memory), not just an external situation. The list of self-report measures of avoidance above is not exhaustive, but it does provide a framework for understanding the idea put forth by Hermans et al., that remembering memories in less specific ways, might form part of a wider repertoire of avoidance strategies, now discussed.

\section{Evidence that reduced memory specificity is part of a repertoire of avoidance}

strategies. Hermans et al. (2005) suggested that remembering less specifically might be part of a wider repertoire of avoidant coping strategies amongst psychologically healthy individuals. That is, people who tend to remember less specifically, compared to those who remember more specifically, would tend to self-report that they use higher levels of avoidance strategies in coping with negative thoughts, emotions, and situations. They tested this idea in a group of 16to17-year-old secondary school students. Participants completed a written AMT and several selfreport measures of avoidance: the AAQ, CBAS, and WBSI. Consistent with their hypothesis, they found negative correlations between memory specificity and each of the avoidance 
measures. That is, the fewer specific responses a participant gave on the AMT, the more highly they scored on the avoidance measures. A more nuanced view of the relationship between memory specificity and CBAS scores emerged when its subscales were examined, however. Participants who provided fewer specific responses on the AMT were more avoidant on the behavioural avoidance subscales of the CBAS, but not the cognitive subscales. There were no significant correlations between categoric or extended AMT responses and avoidance measures. Hermans et al.'s results are consistent with the view that remembering less specifically is an avoidance strategy.

It is important to note however, that there have been some findings that have failed to replicate this basic relationship between overgeneral memory and avoidance. Debeer et al. (2011) reported (unpublished) work that failed to replicate Hermans et al.'s significant results in a sample of undergraduate students. Similarly, Gibbs and Rude (2004) reported no significant associations between avoidance measures and the number of categoric memories on the AMT or the number of specific situations depicted in personal essays.

\section{Evidence that reduced memory specificity is related to less distress following a}

stressful situation. Further support for the functional avoidance role of remembering less specifically comes from research indicating people who retrieved fewer specific memories on the AMT experienced less negative emotion after a stressful event than people who retrieved a higher number of specific memories (Raes et al., 2003). Undergraduate participants completed an AMT and were assigned to either a high-specific group (who gave specific response for each of the ten AMT cues) or low-specific group (who gave six or fewer specific responses). Participants were then randomly assigned to a high-frustration (impossible puzzle task) or lowfrustration (easy puzzle task) condition. Immediately following the puzzle task, high-specific participants reported greater subjective distress in the high-frustration condition compared to low-specific participants. They also reported thinking back to the task more often and experiencing those thoughts as distressing. Based on these findings, Raes et al. proposed that if a person remembers fewer specific memories, then they are less sensitive to negative events, or in other words, reduced memory specificity may be an avoidant coping strategy. Their findings were replicated in a follow up study and were also extended to show that high- and low-specific participants did not differ in their emotion reactions to a positive event (Raes et al., 2006). It is worth noting that Raes et al.'s (2006) participants were selected on the basis of low vs. high 
numbers of specific AMT responses rather than the number of general responses. As they discussed, this indicated that it is not necessarily the tendency to remember in an overgeneral way, but rather to omit responses altogether, that is a benefit to the regulation of emotions in negative situations. Thus, Raes et al. (2006) were drawing attention to the potential difference between the absence of specific memories and the presence of overgeneral memories.

Hermans et al. (2008) extended Raes et al.'s $(2003$, 2006) research investigating the emotion-regulation account of reduced memory specificity by using a real-world stressor and examining change in associated distress over an extended time period. They hypothesised that amongst people who were negatively affected by a stressor, those who were less specific would recover faster from the associated distress. Undergraduate participants were selected who indicated on a questionnaire that they performed worse than expected on a practice exam and who thought their study effort was worthy of a better grade. In short, they were students who were "negatively surprised" (Hermans et al., 2008, p. 788) by their exam results. Participants also completed the Impact of Events Scale, (IES; Horowitz, Wilner, \& Alvarez, 1979) in which they rated their subjective stress in relation to a personally chosen life event that happened during the past week. They also rated the extent to which their wellbeing was negatively impacted by their exam results on an author-created scale based on the IES (Impact of Exams Questionnaire; IExQ). At time 2 (two weeks after first assessment), participants completed these measures again, the AMT, and a trait anxiety measure. At time 3 (nine weeks after first assessment), participants completed the IExQ and IES. A reduction in IES and IExQ from times 1 to 3 was associated with fewer specific AMT responses. Similarly, IExQ change scores from times 1 to 2 were associated with fewer specific AMT responses. That is, participants who reported fewer specific memories on the AMT tended to report a greater reduction in distress related to the exams (IExQ scores) and personally chosen life event (IES) scores across time. These associations remained when trait anxiety was controlled. The authors noted that IExQ and IES scores were not significantly correlated with the number of AMT specific memories at each individual time point. Hermans et al. did not report the association between study variables and overgeneral memories. They concluded that a less specific retrieval style is protective against the negative emotional consequences of a stressful event, in line with Raes et al.'s (2003) findings. Their study supports the affect-regulation account of remembering less specifically. However, Hermans et al. warned of a too-simplistic view of this phenomenon: 
We believe that the general view of reduced memory specificity as being harmful or even pathogenic is too black-and-white. For many persons, the ability to reduce memory specificity in a selective and flexible way might be a natural and healthy way of coping. It might be favourable to not be too specific about all our memories all the time. The ability to reduce or augment memory specificity in a plastic and discriminatory way is part of a larger set of healthy coping strategies. (p. 791)

The idea that there are contexts in which a healthy person might "reduce or augment" the specificity of their autobiographical memories is in line with Raes et al.'s $(2003,2006)$ findings (discussed previously) in which low-specific individuals experienced less distress after a failureexperience task compared with high-specific individuals, but that there was no differences between the high- and low-specific individuals in their reactions to a success-experience task.

These findings from Hermans et al. (2008) and Raes et al. (2003, 2006) are evidence that reduced memory specificity as an avoidant strategy, at least in non-clinical individuals, is used flexibly according to circumstances. This flexibility idea was greatly extended in future research (e.g., Debeer et al., 2011), discussed in the next section.

\section{Evidence that Functional Avoidance is Context-Dependent: The Role of Stress}

The CaR-FA-X model's (Williams et al., 2007) emphasis on the link between overgeneral memory and psychopathology meant that some aspects of the model relating to overgeneral memory in psychologically healthy people were relatively underdeveloped. In particular, and of central relevance to this thesis, is the CaR-FA-X model's description of the flexibility of functional avoidance. Some research since its publication has addressed this issue (Debeer et al., 2011, 2012, 2013), focusing on two related ideas. First, psychologically healthy people who tend to use avoidance strategies to cope with problems might tend to use overgeneral memory as one of those strategies (Hermans et al., 2005). In other words, overgeneral memory may be "part of a larger (cognitive) avoidant repertoire" (Debeer et al., 2013, p. 74).

Second, the use of overgeneral memory as an emotion-regulation strategy may be "context-dependent" (Debeer et al., 2011), that is, it is only used when needed. Debeer et al. argued that the contexts in which it occurs are when the person is under stress. The meaning of the word stress is important here. In general, stress refers to experiences that are emotionally and 
physiologically challenging (McEwen, 2007). An acute stress response consists of activation of physiological systems that rouse an organism to action (Gray \& McNaughton, 2000). The evolved function of this system is survival: a way of responding adaptively to perceived threat. In humans, the physiological systems underlying this response may be activated by psychosocial factors such as living in an adverse environment, or conflict within a relationship (McEwen, 2007). In the case of Debeer et al.'s research, stress was induced in the laboratory by using a stressful public-speaking task (Debeer et al., 2012) or leading participants to believe that autobiographical remembering had the possibility of arousing negative emotions (Debeer et al., 2011). These laboratory stressors were purported analogues to the real-world contexts in which people higher on avoidance engage in functional avoidance.

In summary, elaborations to the functional avoidance mechanism of the CaR-FA-X model have investigated the idea that the tendency to engage in overgeneral remembering is elicited in psychologically healthy people who tend to use avoidant coping strategies and have cause to regulate negative emotion because the context demands it. In other words, if a person tends to use avoidance as a way to cope in stressful situations, then they are more likely, when under stress and as compared to those who are less reliant on avoidance, to exhibit overgeneral memory.

\section{Interaction between avoidance and perception of remembering as threatening. A}

key finding underpinning this thesis is that high avoiders retrieve fewer specific memories on the AMT than low avoiders (Hermans et al., 2005). This raised the possibility that remembering less specifically might be part of a wider repertoire of avoidance strategies. Other research (Hermans et al., 2008; Raes et al., 2006) suggested a more nuanced view, that reducing the specificity of autobiographical memory as an avoidance strategy is deployed flexibly depending on context.

This idea was investigated by Debeer et al. (2011) who noted results from a study with undergraduate psychology students (unpublished) in which the association between avoidance and memory specificity was not found. This was at odds with Hermans et al.'s (2005) findings for secondary school students. Debeer et al. attempted to explain these discrepant findings by suggesting that the different samples (secondary school vs. undergraduate students) experienced the study procedure differently in terms of the amount of accompanying stress. They argued that the secondary school student participants, due to their unfamiliarity with psychological testing, may have experienced the study procedure as stressful. The undergraduate psychology student 
participants on the other hand, due to their familiarity with psychological testing, may not have experienced it as stressful. Debeer et al.'s explanation is in line with the conception of stress advanced by Lazarus, DeLongis, Folkman, and Gruen (1985) who stated that everyday life events interact with person variables such as mastery, sense of control, and interpersonal trust to produce stress. In other words, a person's subjective appraisal of an event is key to the stress response.

This observation, that stress (vs. no stress) might be important with respect to the relationship between avoidant coping and overgeneral memory prompted Debeer et al. (2011) to suggest that people who use avoidant strategies may engage in reduced memory specificity in a “context-dependent" (p. 1501) way. In a test of this idea, they asked undergraduate participants to complete two Minimal-instructions AMTs (Mi-AMT; Debeer et al., 2009). Participants also completed the behavioural subscales of the Cognitive Behavioral Avoidance Scale (CBAS-B; Ottenbreit \& Dobson, 2004). In the "neutral" condition the second Mi-AMT was described to participants as being for the purposes of reliability testing. In the "threat" condition, participants were told the second Mi-AMT "could elicit more negative, emotionally painful memories" (Debeer et al., p. 1502). Thus, the threat condition constituted a stressor to the participants. There was no relationship between CBAS-B and proportion of specific or categoric memories on the first Mi-AMT. On the second, Mi-AMT, however, CBAS-B was significantly negatively correlated with proportion of specific memories and significantly positively correlated with proportion of categoric memories. In other words, the predicted interaction between stress and avoidance emerged. In addition, participants who self-reported higher levels of avoidance showed a significantly greater reduction in specific responding (from first to second Mi-AMT) in the threat condition compared to the neutral condition. Participants who self-reported lower levels of avoidance showed no such difference between conditions. The authors argued that these results indicated reduced memory specificity is activated when remembering itself is perceived as threatening by people who tend to use avoidant coping more generally.

Interaction between avoidance and stress. As discussed above, Debeer et al. (2011) found that when a person who uses avoidant coping perceives remembering as threatening, the specificity of their autobiographical memories is reduced. They argued that the threat condition in their study established a context in which the avoidant coping strategy of remembering less specifically was activated, but only for high-avoiders. Broadly speaking, the threat condition 
induced a stressful context in comparison to the neutral, non-stressful, condition. But are there other (stressful) contexts in which this strategy may be activated?

To address this question, Debeer et al. (2012) investigated the role of social stress in the relationship between avoidant coping and memory specificity. They selected undergraduate participants such that they covered the full range of depression scores on the Beck Depression Inventory II (BDI-II; Beck, Steer, \& Brown, 1996). Participants were administered the AMT and then underwent a stress induction (involving a public speaking task), then completed another AMT (approximately 10 minutes after the stressor) and a delayed AMT (approximately 40 minutes after the stressor). Participants' cortisol levels were measured multiple times during the procedure, and they also completed the Mainz Coping Inventory (MCI; Krohne et al., 2000), a measure of cognitive-avoidance and habitual vigilance coping strategies. The researchers restricted their analyses to a "distressed group" of participants who had elevated cortisol levels post-stressor (that is, those for whom the stressor actually induced stress). In this distressed group, there was a significant positive correlation between cognitive-avoidance (but not vigilance) and change in AMT proportion of categoric memories pre- to immediately poststressor. That is, participants in the distressed group who were higher on cognitive avoidance exhibited a greater increase in proportion of categoric AMT responses pre- to post-stressor compared to those lower on cognitive avoidance. This remained significant when controlling for BDI-II scores. There was no correlation between avoidance or vigilance and change in proportion of specific responses. There were no significant correlations between cognitive avoidance or habitual vigilance and change in proportion of specific or categoric memories preto delayed-AMT. The authors argued that these results are evidence that in times of stress, people high on avoidance regulate their emotions by remembering in an overgeneral way.

Further investigation of the context-dependent nature of autobiographical memory specificity was carried out by Debeer et al. (2013). They hypothesised that if high avoiders use functional avoidance, then by priming an avoidance (vs. approach) schema, they could induce them to remember in a less specific way (as compared to low avoiders). The approach- and avoidance-priming procedure consisted of having participants unscramble sentences relating to approaching and avoiding challenging situations, respectively. Before and after the priming procedure, participants completed an alternative task to the AMT for measuring specificity/overgenerality, the Sentence Completion for Events From the Past Test-Forced 
Choice version (FC-SCEPT; Raes, Watkins, Williams, \& Hermans, 2008). The measure of avoidance used was the White Bear Suppression Inventory (WBSI; Wegner \& Zanakos, 1994), a measure of thought suppression. There was a significant reduction in memory specificity from pre- to post-priming for the group as a whole. There was no differential effect of priming condition (approach vs. avoidance) on the reduction in memory specificity. Avoidance, but not condition (the induction of avoidance vs. approach schema), was a predictor of reduction in memory specificity pre- to post-priming and memory specificity post-priming. To explain the nonsiginficant effect of condition, the authors suggested the sentence-unscrambling task referred to challenging situations which could have been confronting to high-avoiders irrespective of condition and because it was presented as a language test (and were therefore experienced as stressful). In other words, rather than differentially activating approach and avoidance schemas, both conditions may have activated an avoidance schema or been experienced as stressful by high avoiders. This explanation is consistent with Debeer et al.'s (2012) results that showed an increase in overgeneral responding on the AMT pre- to post-stressor.

\section{Functional Avoidance in Clinical and Non-Clinical Groups}

It has been suggested that CaR-FA-X mechanisms (e.g, functional avoidance) may differ in clinical and non-clinical groups (Gutenbrunner, Salmon, \& Jose, 2017b; Sumner, 2012; Sumner et al., 2014) but there is a paucity of research addressing this; further investigation of the relationships between overgeneral memory, stress, and avoidance in depressed vs. non-depressed samples is warranted. Furthermore, whereas the CaR-FA-X model postulates an etiological role of functional avoidance in depression without reference to stress per se, more recent elaborations of the functional avoidance mechanism describe its operation in psychologically healthy (nondepressed individuals) as being modulated by stress. There is therefore a need for investigation of the relationship between these variables in depressed and non-depressed samples.

\section{Evidence against Functional Avoidance}

There are several findings that do not support — in fact, run contrary to - the idea that overgeneral memory is an avoidance strategy. For example, in undergraduate samples, trait thought suppression was found to be associated with faster recall of episodic (i.e., specific) memories (Neufeind, Dritschel, Astell, \& MacLeod, 2009), and suppressing thoughts of a negative video clip was found to be associated with recall of fewer overgeneral memories on a subsequent AMT (Phung \& Bryant, 2013). In other research, dysphoric (as opposed to 
nondysphoric) participants asked to suppress a negative childhood memory (a laboratory analogue to real-world functional avoidance) were faster to recall other specific negative memories on the AMT compared to those not asked to engage in suppression (Dalgleish \& Yiend, 2006). In a procedure where participants were given unlimited time to complete the AMT, abuse symptoms and PTSD predicted greater specificity, opposite to the predictions of the CaR-FA-X model (Bunnell and Greenhoot, 2012). These findings that run opposite to what would be predicted if overgeneral memory were part of an avoidance repertoire highlight that the relationship between these variables is far from clear and that further research is needed.

\section{Summary}

As I stated above, the flexibility concept associated with the functional avoidance mechanism of the CaR-FA-X model was underdeveloped by Williams et al. (2007). They and others (e.g., Hermans et al., 2008) described a distinction between adaptive and maladaptive forms of functional avoidance. The adaptive form is said to be flexible: The person is able to modulate the specificity of remembering whereby sometimes specific memories are remembered, and other times general memories are remembered. The maladaptive form is inflexible: The person has generalised the strategy to all of the autobiographical memory knowledge base, habitually remembering general memories rather than specific. However, with respect to the adaptive, flexible form of functional avoidance, the circumstances surrounding when functional avoidance is used was not understood (the underdevelopment I refer to above). The Debeer et al., (2011, 2012, 2013) studies reviewed above helped to elucidate this. By introducing the notion of the context-dependent nature of the relationship between avoidant coping and overgeneral memory, they provided a way to understand the flexibility of the functional avoidance mechanism. Overall, the evidence suggests that functional avoidance may only be used by those people who (a) tend to use avoidant coping and (b) are under stress. In other words, stress is the context in which functional avoidance occurs.

I use the term stress broadly here. In the studies reviewed in this section, methods included inducing participants to believe that an Mi-AMT might elicit negative memories (Debeer et al., 2011), having participants engage in a stressful public-speaking exercise (Debeer et al., 2012), and having participants unscramble sentences pertaining to challenging situations (Debeer et al., 2013). It is unclear if each of these conceptions of stress can be likened to one another. Nevertheless, results from these studies converge on the idea that for high avoiders, 
stressful situations induce overgeneral memory (increased general remembering and decreased specific remembering), at least in the short term. It is an open question as to what types of stress can influence the relationship between avoidant coping and overgeneral memory.

The primary aim of this thesis is to continue the line of research summarised above by investigating the role of stress as it occurs in people's daily lives (as opposed to stress induced in the laboratory) in the relationship between avoidance and overgeneral memory. In brief, it is motivated by the following. First, there are few studies in this area, so examination of the relationships between stress, avoidance, and overgeneral memory are necessary for the purposes of replication. In particular, there is some research that does not support the idea that overgeneral memory is an avoidance strategy; i.e., the basic bivariate relationship between avoidance and overgeneral memory is not clear. Second, existing research has used laboratory-induced stressors, but the applicability of these results to stress as it is experienced in people's daily lives is unknown. Third, investigation of the relationships between these variables is necessary in clinical (vs. non-clinical) populations.

A secondary aim of this thesis relates to the emotional nature of specific vs. general memories, discussed in the next section.

\section{The Emotional Nature of Specific vs. General Memories}

One of the key ideas I have discussed in this chapter is that psychologically healthy people may be motivated to avoid specific memories because specific memories carry with them potentially unpleasant emotions. In this section, I describe research that presents a challenge to this idea. In doing so, I elucidate a secondary research aim of this thesis: to investigate whether avoidant coping is associated with the avoidance of general memories.

In a previous section, I described how specific memories in the self-memory system are considered representations of emotional, experiential outcomes of plans for goal attainment (Conway \& Pleydell-Pearce, 2000). In describing specific memories this way, Conway and Pleydell-Pearce provided a way of understanding why a person would want to avoid an unpleasant specific memory: Remembering it runs the risk of re-instantiating the associated negative emotions and destabilising the ongoing goals of the working self. As Conway and Pleydell-Pearce state: "Clearly it makes some adaptive sense to terminate a memory search that threatens to access knowledge that may destabilize the goals of the working self and cast the whole system into turmoil" (p. 282). The situation may be more complicated than this, however. 
Consider Williams' (1996) descriptions of the emotional impact of remembering specific vs. categoric negative memories: A "sharp pain” (p. 261) is the impact of unpleasant specific memories. A "dull ache” (p. 261), albeit less severe than a sharp pain, is the impact of unpleasant categoric memories. There is an emphasis in much of the overgeneral memory literature on the negative emotional consequences of specific memories, but as alluded to in Williams' descriptions, the negative emotional consequences of general memories should also be considered. It is not simply that specific remembering causes negative emotions and general remembering does not. Rather, as Williams states, both types of remembering have potentially aversive accompanying emotions.

Williams's (1996) conjecture about the aversive nature of specific and general memories is interesting in light of work by Bunnell and Greenhoot (2017). In separate studies with children (8-17 years) and undergraduate students, they examined how general and specific memories influenced emotional state. The child and undergraduate studies had the same design. In one condition, participants listened back to recordings of their own previously-generated general memories. In another condition, participants listened to their previously-generated specific memories. Positive and negative affect was measured before and after memory exposure. Children who listened to their general memories reported higher positive affect than those that listened to their specific memories, indicating some support for the functional-avoidance account of overgeneral memory. That is, for this participant group, remembering general memories rather than specific memories seemed to have some adaptive benefit for mood-regulation. On the other hand, there was no effect of condition in undergraduates. That is, there was no difference in the influence of specific and general memories on affect in adults, at odds to what would be expected if specific memories are more aversive than general. These findings are further indication that, at least in undergraduates, specific and general memories are comparable in terms of their affective impact.

Based on these ideas, I propose that the aversive nature of general memories has not been adequately addressed in the functional avoidance literature. In particular, the idea that remembering general memories may have a comparable emotional impact to specific memories raises the possibility that people who are high on avoidant coping may wish to avoid remembering general memories, in just the same way that they may wish to avoid remembering specific memories. In short, general memories are a potential target for avoidance, and as such, 
avoiding general memories may be part of a wider repertoire of avoidant coping strategies. Note that this argument is exactly the same logic that has been presented elsewhere regarding specific memories (Debeer et al., 2011; Hermans et al., 2005).

In summary, whereas much previous research has been based on the idea that specific memories are a target for avoidance, there is also a reason to examine general memories: In addition to specific memories, general memories may also be experienced as aversive.

\section{Research Questions}

The aim of this thesis was to systematically address several important outstanding questions regarding the idea that remembering less specifically is part of a wider repertoire of avoidance strategies. I have organised these aims into six specific research questions. The first three questions overlap, all pertaining to the relationships between AMT indices, stress, and avoidance; I therefore group these together as Research Questions 1a, 1b, and 1c.

\section{Research Question 1a: What is the bivariate relationship between avoidance and} overgeneral memory in non-clinical samples? Very few studies have investigated the relationships between overgeneral memory and avoidance, and replication of these few findings is necessary because they are inconclusive. Of particular note are the findings that in a sample of high school students, higher avoidance was associated with lower memory specificity (Hermans et al., 2005). However, Debeer et al. (2011) and Gibbs and Rude (2004) reported no significant associations between avoidance and overgeneral memory in undergraduate students. Thus, given these inconsistent findings, this research question is underpinned by the need for replication. This research question was investigated in Studies 1, 2, and 4.

\section{Research Question 1b: Does self-reported stress moderate the relationship between} avoidance and overgeneral memory in non-clinical samples? In an attempt to explain the contradictory findings described above, Debeer et al. (2011) suggested that stress played a moderating role in the relationship between avoidance and overgeneral memory such that high avoiders' memory specificity was only reduced when they were under stress. There are few studies investigating this however, and moreover, research to date has used laboratory stressors, rather than self-reported levels of daily stress "outside the lab". For example, these inductions involved creating artificial situations over relatively acute time periods, and as discussed earlier, they were purported analogues to the real-world contexts in which a person higher on avoidance might engage in functional avoidance (Debeer et al., 2011). It is an open question as to whether 
these findings can be replicated if more real-world, chronic stress is considered. Thus, a further elaboration to Research Question 1a is: Do high-avoiders exhibit reduced memory specificity only when they self-report high levels of stress? In other words, does self-reported stress moderate the relationship between avoidance and memory specificity? This research question was investigated in Studies 1, 2, and 4.

\section{Research Question 1c: What are the relationships between avoidance, overgeneral} memory, and stress in a clinical sample? The last of the research questions pertaining to memory specificity being part of a repertoire of avoidance strategies is predicated on the idea that CaR-FA-X mechanisms may be different in clinical vs. non-clinical populations (Gutenbrunner et al., 2017b; Sumner, 2012; Sumner et al., 2014; Williams et al., 2007). Investigation of key relationships among variables (i.e., overgeneral memory, stress, avoidance) in clinical as well as non-clinical samples is therefore warranted. This research question was investigated in Study 3.

\section{Research Question 2: Is remembering a lower proportion of general memories on} the AMT-R associated with higher avoidance? Although specific memories may elicit more negative emotions than nonspecific memories, both types of memories can be aversive (Williams, 1996). To date, research into functional avoidance has used tasks which include an explicit instruction to remember specific events (e.g., AMT), or no such instruction (e.g., MiAMT). In light of Williams's idea that general as well as specific memories can be aversive, more complete tests of hypotheses pertaining to the relationship between avoidance and autobiographical memory performance would need to include a test that contains an explicit instruction to remember general memories. By including such a test, the following question can be addressed: Is any reduction in performance on the AMT exhibited by high avoiders restricted to AMT performance, or is there also a reduction in other tests of autobiographical memory performance? In Study 1, I therefore investigated the relationship between avoidance and performance on the Autobiographical Memory Test-Reversed (AMT-R; Dalgleish et al., 2007), a task in which participants are asked to retrieve general (categoric) memories. This research question was investigated in Study 1.

Research Question 3: Do positive and negative cues elicit memories for positive and negative events, respectively? I used this thesis to carry out a methodological check pertaining to the cues used on the AMT (and AMT-R). The AMT is typically administered with positively 
and negatively-valenced emotion cue words (see Griffith et al., 2012 for a review). There has been an underlying assumption in the overgeneral memory literature that sampling a person's autobiographical memory using positively and negatively valenced cues elicits positive and negative memories, respectively. As Griffith et al. state, however, there may not be direct correspondence between cue and memory valence. The third question therefore was to ask whether positive and negative cues elicit memories for events a person rates as pleasant and unpleasant, respectively. This research question was investigated across all four studies.

\section{Research Question 4: Does rating the pleasantness of events influence overgeneral} memory on the AMT? Based on findings from Studies 1, 2, and 3, a fourth research question emerged. In short, I speculated that the pleasantness rating scale on each AMT trial may have influenced the relationships between overgeneral memory and self-reported avoidance differentially for participants high and low on avoidance. Thus, the fourth research question was aimed at investigating this possibility. This was investigated in Study 4. 


\section{Chapter 2}

\section{Study 1: The Relationship between Avoidance and Memory Specificity in a Non-clinical Sample}

Some wording in this chapter is the same as in Ganly, Salmon, and McDowall (2016). I have modified and added to some wording from the article in the present thesis for the purposes of clarity and elaboration.

\section{Introduction}

In this chapter I describe Study 1 of the present thesis. Study 1 was designed to replicate the method used in Hermans et al. (2005), but with some modifications and extensions. The main difference between Hermans et al.'s method and Study 1 was that in addition to completing the AMT, all participants completed a reversed version of the AMT (AMT-R; Dalgleish et al., 2007) in which participants were asked to retrieve categoric memories as opposed to specific memories.

Study 1 research aims. Study 1 addressed the following research aims. The first aim was to investigate the basic relationship between self-reported avoidant coping and autobiographical memory specificity and overgenerality. The second aim was to investigate the moderating role of self-reported stress in the relationship between avoidant coping and memory specificity and overgenerality. In other words, it was to investigate how stress and avoidant coping interact to predict memory specificity and overgenerality. The third aim of Study 1 was a methodological check to investigate whether positive and negative cues elicited memories for events rated as pleasant and unpleasant, respectively. The fourth aim pertained to carrying out a more complete test of Hermans et al.'s (2005) hypothesis that people who self-reported higher levels of avoidance would remember fewer specific memories. Their methodology involved asking participants to remember specific memories on the AMT and to complete self-report measures of avoidance. This methodology does not take into account the possibility that avoidant participants also perform poorly on a task in which they are asked to remember general memories. The fourth aim, therefore, was to investigate how avoidant coping is related to performance on a 'reversed' version of the AMT (AMT-R; Dalgleish et al., 2007) in which the participant is asked to retrieve general memories. Based on these research aims, several predictions were developed. These aims were addressed in a sample of undergraduate students.

The first prediction concerned the basic bivariate relationship between avoidance 
measures and AMT performance. I predicted that there would be no significant relationship between AMT performance and avoidance measures (hypothesis 1).

I also predicted that stress would moderate the relationships between avoidant coping and performance on the AMT. In particular, compared to those participants lower on self-reported avoidant coping, those higher on self-reported avoidant coping will exhibit a lower proportion of specific memories and higher proportion of overgeneral memories on the AMT, but this will only emerge when self-reported levels of stress are high (hypothesis 2).

As a methodological check, I asked participants to rate the pleasantness of remembered events on a continuum of extremely unpleasant to extremely pleasant (see Method section). I predicted that mean pleasantness ratings of events remembered to positive cues would be higher than to negative cues. In addition, I predicted that mean pleasantness ratings for events remembered to positive cues would be significantly higher than the midpoint of the scale, and that mean pleasantness rating for events remembered to negative cues would be significantly lower than the midpoint of the scale. I shall refer to these predictions as hypothesis 3.

If, as Williams (1996) suggested, remembering general memories can be aversive, then there should be a negative correlation between avoidance and performance on the AMT-R. That is, higher self-reported avoidance should be associated with a lower proportion of categoric AMT-R responses (hypothesis 4). This prediction is extremely tentative, however; given the inconsistent previous findings regarding the relationship between AMT indices and avoidance, this aim was carried in out an exploratory vein. Also in an exploratory vein, I examined the relationship between avoidance and proportion of specific AMT-R responses.

\section{Method}

The method described herein was based closely on Hermans et al. (2005). Some of the wording in this method section is similar to Ganly et al. (2016). For the purposes of clarity, I do not continually cite Ganly et al.

Participants. Participants were 82 undergraduate psychology students. Participants were recruited in sufficient numbers as to exceed Hermans et al.'s (2005) sample size $(N=60)$ and so there would be approximately 20 participants per counterbalanced cell (AMT/AMT-R $\times$ cue-set 1/cue-set 2, see Materials section). Participants were selected on the basis of English being their first language, and being between 18 and 20 years old. Course credit was awarded for participation. One participant withdrew before completing all the measures, and their partial data 
were excluded from all analyses. Of the 81 participants who completed the procedure, 58 (72\%) were female. Of the 79 participants who reported their age, the mean age was $18.62(S D=0.69)$.

Materials. AMT and AMT-R. Participants completed written versions of the Autobiographical Memory Test (AMT; Williams \& Broadbent, 1986) and Autobiographical Memory Test - Reversed (AMT-R; Dalgleish et al., 2007). But for the instructions given to participants, these tests were administered in very similar fashion, and in the following descriptions, I describe them in tandem, highlighting differences where appropriate. As discussed in Chapter 1, in the AMT, participants are asked to retrieve and report specific memories that cue words reminded them of. The AMT-R, on the other hand, has a modified instruction: Participants are asked to retrieve categoric memories rather than specific memories.

Cues. In both the AMT and AMT-R, cue words were five positively and five negatively valenced cues (e.g., proud, tense) alternating between valences. Because of the administration of two different tests (AMT and AMT-R), two different sets of cues were used; Cue Sets A and B were taken from Sumner, Mineka, and McAdams (2012), and are shown in Appendix A. Cuesets and tests were counterbalanced, leading to four cue-set / test combinations: (1) AMT with Cue Set A followed by AMT-R with Cue Set B, (2) AMT-R with Cue Set A followed by AMT with Cue Set B, (3) AMT with Cue Set B followed by AMT-R with Cue Set A, (4) AMT-R with Cue Set B followed by AMT with Cue Set A.

Instructions. AMT and AMT-R instructions given to participants are in Appendices B and $\mathrm{C}$, respectively. In both the AMT and AMT-R, participants were asked to retrieve a memory that each cue reminded them of. In the AMT, participants were asked to retrieve a specific memory to each cue. Specific memories were defined as those referring to an event that happened on one particular occasion, at a particular time and place, and that lasted less than one day. In the AMT-R, participants were asked to retrieve a categoric memory to each cue, defined as referring to a series of similar events that happened at different times; in other words, a category of events. For each test, participants were asked not to use the same memory more than once and that if they couldn't think of a memory, to leave that page blank. In the case of the AMT, participants were asked to retrieve memories that were at least one week old. For each test, an example of a correct and incorrect response was given. After the instructions for each test were read aloud, an opportunity was given to participants to ask questions.

Pleasantness ratings of autobiographical memories. To check that positive and negative 
cues were eliciting memories for positive and negative events, respectively, participants were asked to rate the pleasantness of each remembered event (AMT) or category of event (AMT-R) on a 5-point scale with 1 meaning extremely unpleasant and 5 meaning extremely pleasant. This approach was taken from Geraerts, Dritschel, Kreplin, Miyagawa, and Waddington (2012). Instructions for making the ratings given to participants are shown in appendices B and C.

Response booklets. Participants completed the AMT and AMT-R in separate landscape A5-sized booklets. Booklets were printed single-sided, bound with a single staple in the top left hand corner, and had a cue printed at the top left of each page. The pleasantness scale with anchors was printed at the bottom of each page.

Trials. Each test consisted of two practice trials followed by the ten test trials. Cues used in the practice trials are shown in Appendix A. After the practice trials, another opportunity was given to participants to ask questions about the test. Trials lasted one minute. Practice trial instructions are shown in Appendix D.

Coding. In line with previous research (see Griffith et al., 2012), responses were coded coded as specific if they referred to an event that lasted less than one day, categoric if they referred to a category of events, extended if they referred to an event lasting more than one day, semantic associate if they referred to a semantic associate of the cue, omission if there was no response, and error if the response was future-oriented, incomprehensible, an obvious repetition of a previous memory, or, in the case of the AMT, obviously less than one week old (e.g., the response contained the phrase, "Earlier on in the week ..."). The coding scheme is shown in Appendix E. AMT responses from $25 \%$ of participants, randomly selected and approximately equal in number from each cue-set, were coded independently by a second rater. The same reliability procedure was followed for the AMT-R. Cohen's Kappas were calculated, and interrater agreements for AMT and AMT-R responses were acceptably high, $\mathrm{K}=.87$ and .74, respectively. Disagreements were resolved by the primary rater (TG).

Questionnaire measures. Self-report measures of avoidance, depression, and stress were administered in an A4-sized booklet printed double-sided.

Cognitive Behavioral Avoidance Scale (Ottenbreit \& Dobson, 2004). The CBAS is a selfreport measure of cognitive and behavioural avoidance in social and non-social domains. Its 31 items are divided into 4 subscales tapping behavioural-social (e.g., "I avoid attending social activities"), behavioural-non-social (e.g., "I avoid trying new activities that hold the potential for 
failure"), cognitive-social (e.g., "I try not to think about problems in my social relationships"), and cognitive-non-social (e.g., "I avoid making decisions about my future") avoidance.

Participants are asked to rate how true, in general, each statement is for them on a 5-point Likert scale from 1 (not at all true for me) to 5 (extremely true for me). The CBAS total score is created by summing item responses. Minimum and maximum possible scores are 31 and 155 , respectively, with higher scores indicating higher levels of cognitive-behavioural avoidance. Minor wording changes to the CBAS were made to adapt it for a New Zealand context (in instruction and items, changing "school" to "university") and to reduce the leading nature of some items (items that used the wording "I fail to" were change to "I do not"). Cronbach's alpha for the full scale score in Studies 1, 2, 3, and 4 indicated good internal consistencies, $\alpha=.94, .92$, .91 , and .90 , respectively. In the current thesis, I report the CBAS total score, as I did in Ganly et al. (2016). In addition, because some previous research has examined the behavioural subscale (CBAS-B; e.g., Debeer et al., 2011), in this thesis I also report the CBAS broken down into CBAS-B and CBAS-C (cognitive) subscales.

Acceptance and Action Questionnaire - II (Bond et al., 2011). The AAQ-II is a selfreport measure of experiential avoidance. One of the aims of Study 1 was to replicate Hermans et al. (2005) who used the original AAQ (Hayes et al., 2004). According to Bond et al., however, the AAQ had several problems such as complicated items and insufficient internal and test-retest reliability. I therefore opted to administer the updated version, the AAQ-II, which addressed these issues. The AAQ-II consists of seven statements (e.g., "Worries get in the way of my success") that are rated on a 7-point Likert scale from 1 (never true) to 7 (always true). Item responses are summed to create the full scale score. Minimum and maximum possible scores are 7 and 49, respectively, with higher scores indicating higher experiential avoidance. Cronbach's alpha for the AAQ-II in Studies 1, 2, 3, and 4 indicated good internal consistencies, $\alpha=.90, .90$, .85 , and .91 , respectively.

White Bear Suppression Inventory (WBSI; Wegner \& Zanakos, 1994). The WBSI is a self-report measure of an individual's tendency to suppress negative thoughts. It consists of 15 statements (e.g., "I have thoughts that I try to avoid") that are rated on a 5-point Likert scale from 1 (strongly disagree) to 5 (strongly agree). Item responses are summed to create the full scale score. Minimum and maximum possible scores are 15 and 75, respectively, with higher scores indicating higher levels of thought suppression. Cronbach's alpha for the WBSI in Studies 
1, 2, 3, 4 indicated good internal consistencies, $\alpha=.91, .91, .75$, and .92 , respectively.

Depression Anxiety Stress Scales 21 (DASS; Lovibond \& Lovibond, 1995). The DASS is a self-report measure of depression, anxiety, and stress that a person has experienced over the past week. It consists of three subscales (depression, anxiety, stress) of seven items each. I administered the short, 21-item version of the 42-item DASS. In this thesis, my primary interest was participants' scores on the Stress and Depression subscales, and so findings for anxiety are not reported. As I aimed to recruit a psychologically healthy sample for this study, the DASS depression subscale was used to check the mean depression score was within the "normal" (see below) range. DASS depression and stress subscales contain seven items each with statements relating to feelings of depression (e.g., "I couldn't seem to experience any positive feeling at all"), and stress (e.g., "I found it hard to wind down"), respectively. Participants rate how much each statement applied to them over the past week on a 4-point scale from 0 (Did not apply to me at all) to 3 (Applied to me very much, or most of the time). Subscale scores are calculated by summing item responses for the subscale and multiplying by 2 (so that scores are comparable with the full 42-item scale). Higher depression and stress scores indicate higher levels of depression and stress, respectively. The DASS defines "normal", "mild", "moderate", "severe", and "extremely severe" labels for characterising scores relative to the full range of scores in the population. Cronbach's alphas for the depression and stress subscales in Study 1 were $\alpha=.91$ and .81, respectively; in Study 2, $\alpha=.88$ and .85, respectively; in Study $3, \alpha=.87$ and .67 , respectively; and in Study $4, \alpha=.88$ and .80, respectively; indicating acceptable to good internal consistencies.

Procedure. The study was approved by the School of Psychology Human Ethics Committee under the delegated authority of Victoria University of Wellington's Human Ethics Committee. An approximately equal number of participants were randomly allocated to each counterbalanced condition with the constraint that the proportion of males to females in each condition was approximately equal. Participants took part in groups of one to four. They were greeted by the experimenter and given an information sheet. The information sheet introduced the study as investigating "how people remember" and stated participants would "respond to statements about how you deal with everyday situations and hassles and provide information about your mood and thinking style". It also stated that they would write descriptions of events from their life, that completion of the questionnaires constituted consent, and that they were free 
to withdraw at any point.

The AMT and AMT-R (counterbalanced as per the scheme described above) were administered first. Following the AMT and AMT-R, participants were administered a written verbal fluency test (not reported further in the present thesis), and were then given a questionnaire booklet which included the CBAS, AAQ-II, WBSI, and DASS. It also included the brooding and reflection subscales of the Rumination Response Scale (RRS; Treynor, Gonzalez, $\&$ Nolen-Hoeksema, 2003) but these are not reported further. Participants received a written debriefing statement upon completion, and any questions they had about the study were answered.

\section{Results}

Data-analytic approach. The data analytic approach involved carrying out multiple statistical tests. In such situations, the potential for type I errors increases. That is, the chance of detecting "false positives" (incorrectly rejecting a true null hypothesis) increases as a function of the number of significance tests. Alpha adjustment is sometimes advocated so that the potential for type I errors is reduced; this is achieved by setting a more stringent value for alpha (see Cumming, 2012; Cumming \& Calin-Jageman, 2016; Rothman, 1990, for discussions). Following recommendations from Cumming (2012), however, rather than adjusting alpha, I opted to carry out holistic interpretation of the overall results by examining effect sizes and associated $95 \%$ confidence intervals (CIs). As per convention in this field, however, I also provide $p$ values for significance tests. By presenting CIs and indications of the value of $p$ in the traditional manner, I hoped to make statistical tests as interpretable by the reader as possible.

Some previous research has reported non-normal distributions in specificity and overgenerality as measured using the AMT (e.g., Hermans et al., 2005). Bootstrapping has been recommended as an approach to dealing with non-normality (Field, 2013; Field \& Wilcox, 2017; Howell, 2013), and with this in mind, I bootstrapped confidence intervals (CIs) using 2000 samples (bias-corrected and accelerated; $\mathrm{BCa}$ ). The likely value in the population for a particular estimate lies within the upper and lower bounds of the CI.

Missing data. In accordance with recommendations from Lovibond and Lovibond (1995), missing data in three DASS items were imputed with the mean score from the particular subscale. The one item non-response each in the WBSI and CBAS (cognitive-social subscale) were imputed using the same technique. The two missing pleasantness ratings for 
autobiographical memories were imputed by taking the particular participant's average pleasantness rating for the subset of cues of that valence.

Descriptive statistics. $\boldsymbol{A M T}$ and $\boldsymbol{A M T}$ - $R$. Descriptive statistics for Study 1 variables are shown in Table 2.1. Psychometric analyses of the AMT (e.g., Griffith et al., 2009; Heron et al., 2012) has shown it to have a one-factor structure, so responses to positive and negative cues were collapsed. A proportional index of AMT specificity excluding omissions for each participant was created: Proportion of specific responses $=$ number of specific responses/(10 total number of omissions). I also created a proportional AMT overgenerality index for each participant: Proportion of overgeneral responses $=$ (number of categoric responses + number of extended responses $) /(10$ - total number of omissions $)$. I excluded omissions in the calculation of these indices because, as discussed in the introduction, their meaning is unclear.

For the AMT-R, a proportional index of AMT-R categoric memories was created: Proportion of categoric responses $=$ Number of categoric responses/ $(10-$ total number of omissions). In keeping with previous research using the AMT-R (Dalgleish et al., 2007), an index of AMT-R specificity was also created. This was a proportional index of specificity excluding omissions created in the same way as in the AMT. 
Table 2.1

Descriptive Statistics for Study 1 Variables

\begin{tabular}{|c|c|c|c|c|}
\hline Variable & $M$ & $S D$ & Minimum & Maximum \\
\hline AMT proportion spec & .81 & .26 & .00 & 1.00 \\
\hline AMT proportion OG & .05 & .08 & .00 & .30 \\
\hline AMT-R proportion cat & .81 & .28 & .00 & 1.00 \\
\hline AMT-R proportion spec & .11 & .25 & .00 & 1.00 \\
\hline AMT pleasantness pos & 4.04 & 0.55 & 2.33 & 5.00 \\
\hline AMT pleasantness neg & 1.75 & 0.40 & 1.00 & 2.60 \\
\hline AMT-R pleasantness pos & 4.09 & 0.54 & 2.00 & 5.00 \\
\hline AMT-R pleasantness neg & 1.94 & 0.47 & 1.20 & 3.60 \\
\hline \multirow[t]{2}{*}{ DASS depression } & 9.27 & 9.83 & 0.00 & 42.00 \\
\hline & Normal & & & \\
\hline \multirow[t]{2}{*}{ DASS stress } & 12.96 & 8.44 & 0.00 & 36.00 \\
\hline & Normal & & & \\
\hline AAQ-II & 21.70 & 9.08 & 7.00 & 49.00 \\
\hline CBAS total & 64.54 & 20.10 & 35.00 & 114.00 \\
\hline CBAS-B & 28.32 & 10.35 & 14.00 & 56.00 \\
\hline CBAS-C & 26.22 & 11.67 & 18.00 & 67.00 \\
\hline WBSI & 50.67 & 11.66 & 17.00 & 71.00 \\
\hline
\end{tabular}

Note. AMT and AMT-R indices are proportions excluding omissions. AAQ-II $=$ Acceptance and Action Questionnaire II; AMT = Autobiographical Memory Test; AMT-R = Autobiographical Memory Test - Reversed; CBAS $=$ Cognitive Behavioral Avoidance Scale; CBAS-B = CBAS behavioural subscale; CBAS-C = CBAS cognitive subscale; DASS = Depression Anxiety Stress Scales 21-item version; Neg = negative; Normal = DASS severity label; OG = overgeneral responses; Pos $=$ positive; Spec $=$ specific responses; WBSI $=$ White Bear Suppression Inventory. 
Depression, stress, and avoidance. As shown in Table 2.1, DASS depression and stress scores fell within the "normal" severity range indicating that levels of depression and stress in the sample as a whole were below the typical severity of those that might seek help (Lovibond \& Lovibond, 1995).

\section{Hypothesis 1: Relationships between avoidance and AMT indices. Bivariate} correlations between study measures are displayed in Table 2.2 along with their associated confidence intervals. The only significant AMT-avoidance relationship was a negative correlation between CBAS scores (CBAS total and its CBAS-C subscale) and proportion of AMT overgeneral responses; that is, participants who self-reported higher levels of avoidance on CBAS total and the CBAS-C subscale reported a lower proportion of overgeneral AMT responses. This indicated partial support for Hypothesis 1 that there would be no significant relationship between avoidance and AMT indices.

\section{Hypothesis 2: Stress as a moderator of the relationship between avoidance and}

AMT indices. In Ganly et al. (2016), I did not report tests of the moderating role of stress on the relationships between AMT indices and avoidance measures when there was no significant bivariate relationship between the particular avoidance measure and AMT index. The absence of a significant correlation between two variables does not preclude the possibility that their relationship is moderated by a third variable, however. For this reason, I tested the possible moderating role of DASS stress on the relationship between each AMT index (proportion of specific responses, proportion of overgeneral responses) and each avoidance measure (AAQ-II, CBAS total, CBAS-B, CBAS-C, WBSI). These were tested using hierarchical multiple regressions. For each regression, the particular AMT index (i.e., proportion of specific responses, proportion of general responses) was the outcome, and the predictors were the particular avoidance measure (e.g., AAQ-II), DASS stress, and the particular interaction term (e.g., AAQ-II $\times$ DASS). The particular avoidance measure was added at the first step, stress was entered on the second step, and the interaction term was added on the third step. When predicting proportion of specific responses, the third steps of these models were not significant, $F \mathrm{~s}=0.25$ to $0.77, p \mathrm{~s}=$ .518 to .864 .

When predicting proportion of overgeneral responses, the third steps of the models involving AAQ, CBAS-B, and WBSI were not significant, $F \mathrm{~s}=0.76$ to $1.73, p \mathrm{~s}=.081$ to .522 . The third step of the model involving the CBAS total score was significant, $R^{2}=.11$, adjusted $R^{2}$ 
$=.08, F(3,77)=3.20, p=.028$, but in this model, the CBAS total $\times$ stress interaction term was not significant, $B=-0.00004, \beta=-.43, t(77)=-0.90, p=.493$, BCa $95 \%$ CI [ $-0.0002,0.00003]$. The third step of the model involving the CBAS-C score was significant, $R^{2}=.12$, adjusted $R^{2}=$ $.09, F(3,77)=3.48, p=.020$, but in this model, the CBAS-C $\times$ stress interaction term was not significant, $B=-0.00006, \beta=-.36, t(77)=-.73, p=.469$, BCa 95\% CI [-0.0003, 0.00008]. These results indicated that stress did not moderate the relationship between avoidance and overgeneral memory. 
Table 2.2

Correlations [and 95\% BCa Confidence Intervals] between Depression, Stress, Avoidance, and AMT and AMT-R Indices in Study 1

\begin{tabular}{|c|c|c|c|c|}
\hline Variable & AMT spec & AMT OG & AMT-R spec & AMT-R cat \\
\hline \multirow[t]{2}{*}{ DASS depression } & .05 & -.21 & -.09 & -.01 \\
\hline & {$[-.191, .272]$} & {$[-.362,-.026]$} & {$[-.220, .078]$} & {$[-.232, .173]$} \\
\hline \multirow[t]{2}{*}{ DASS stress } & -.05 & .04 & -.06 & -.02 \\
\hline & {$[-.274, .177]$} & {$[-.179, .250]$} & {$[-.257, .147]$} & {$[-.235, .183]$} \\
\hline \multirow[t]{2}{*}{ AAQII } & -.001 & -.16 & -.05 & .02 \\
\hline & {$[-.218, .221]$} & {$[-.345, .050]$} & {$[-.237, .122]$} & {$[-.169, .212]$} \\
\hline \multirow[t]{2}{*}{ CBAS total } & -.05 & $-.27 *$ & -.06 & .02 \\
\hline & {$[-.287, .194]$} & {$[-.428,-.101]$} & {$[-.235, .145]$} & {$[-.210, .236]$} \\
\hline \multirow[t]{2}{*}{ CBAS-B } & .002 & -.19 & -.04 & .01 \\
\hline & {$[-.219, .207]$} & {$[-.355, .010]$} & {$[-.237 .178]$} & {$[-.209, .226]$} \\
\hline \multirow[t]{2}{*}{ CBAS-C } & -.09 & $-.30 * *$ & -.06 & .03 \\
\hline & {$[-.308, .138]$} & {$[-.468,-.120]$} & {$[-.230, .130]$} & {$[-.211, .226]$} \\
\hline \multirow[t]{2}{*}{ WBSI } & -.09 & -.01 & .0003 & -.05 \\
\hline & {$[-.299, .130]$} & {$[-.215, .195]$} & {$[-.161, .151]$} & {$[-.199, .097]$} \\
\hline
\end{tabular}

$\overline{\text { Note. AMT and AMT-R indices are proportions excluding omissions. AAQII }=\text { Acceptance and }}$ Action Questionnaire II; AMT = Autobiographical Memory Test; AMT-R = Autobiographical Memory Test - Reversed; Cat $=$ Proportion of categoric memories; CBAS $=$ Cognitive Behavioral Avoidance Scale; CBAS-B = CBAS behavioural subscale; CBAS-C = CBAS cognitive subscale; DASS = Depression Anxiety Stress Scales 21-item version. OG = Proportion of overgeneral memories; Spec $=$ Proportion of specific memories; WBSI $=$ White Bear Suppression Inventory.

$* p<.05 . * * p<.01$. 
Hypothesis 3: Pleasantness ratings of autobiographical memories. AMT. To test the hypothesis that positive and negative AMT cues elicited memories for pleasant and unpleasant events, respectively, I used one-sample $t$-tests to test the difference between the midpoint of the pleasantness scale (3) and pleasantness ratings to positive and negative cues. I also carried out paired-samples $t$-tests to test if there was a statistically significant difference between ratings to positive and negative cues. Note that in Ganly et al. (2016), only the paired-samples $t$-tests were reported. The addition of the one-sample t-tests in this thesis constitute a more complete test of the hypothesis.

Pleasantness ratings to positive AMT cues were higher than the midpoint, a statistically significantly mean difference of $1.05, \mathrm{BCa} 95 \%$ CI $[0.93,1.16], t(80)=17.21, p<.001$. Pleasantness ratings to negative AMT cues were lower than the midpoint, a statistically significant mean difference of -1.25 . BCa 95\% CI [-1.33, -1.15], $t(80)=-27.83, p<.001$.

The difference in mean AMT pleasantness ratings to positive and negative cues was 2.29, BCa 95\% CI [2.14, 2.44]. The short CI indicates that on the 5-point pleasantness scale, participants were very likely to rate memories remembered to positive AMT cues higher in pleasantness than those remembered to negative cues. Moreover, given the null hypothesis of no difference between the pleasantness ratings between the two cue valences, this difference was statistically significant, $t(80)=30.84, p<.001$.

$A M T-R$. Pleasantness ratings to positive AMT-R cues were higher than the midpoint, a statistically significantly mean difference of 1.09 , BCa 95\% CI [0.96, 1.21], $t(80)=18.12, p<$ .001 . Pleasantness ratings to negative AMT-R cues were lower than the midpoint, a statistically significant mean difference of $-1.06, \mathrm{BCa} 95 \% \mathrm{CI}[-1.16,-0.96], t(80)=-20.08, p<.001$.

The difference in mean pleasantness ratings between positive and negative AMT-R cues was 2.15 , BCa 95\% CI $[1.98,2.31]$. Similar to the AMT, the short CI around this mean difference indicates participants gave consistently higher pleasantness ratings to positive AMT-R cues than to negative AMT-R cues. Given the null hypothesis of no difference between AMT-R cue valences, this difference was significant, $t(80)=25.76, p<.001$.

These results support the prediction that positive and negative cues would elicit memories for pleasant and unpleasant events, respectively. Further examination of these pleasantness rating data are presented in Chapter 8. 
Hypothesis 4: Relationship between avoidance and AMT-R indices. As can be seen in Table 2.2, correlations between AMT-R indices and avoidance measures all had effect sizes that were all $<.1$ and CIs that included zero. Furthermore, the correlations were statistically nonsignificant. These results did not support the prediction that avoidance would be associated with reductions in performance on the AMT-R.

\section{Discussion}

The first hypothesis was that there would be no significant correlations between avoidance and AMT indices. This was partly supported: The only significant correlations between AMT indices and avoidance measures were significant negative correlation between the CBAS (total score and CBAS-B) and proportion of AMT general memories. This finding is at odds with earlier findings that people higher on avoidant coping exhibit more overgeneral memory; Hermans et al. (2005) found negative correlations between specific memories and avoidance measures (AAQ, CBAS total, CBAS behavioural subscales, and WBSI), whereas I found no correlations. In addition, the direction of the significant correlations I found are at odds with the idea that people higher on avoidance would remember a higher proportion of general memories, rather, they suggest that people higher on avoidance remember a lower proportion of general memories. The confidence intervals for these significant effects are relatively wide, consistent with between a small and medium sized effect (Cohen, 1992).

The second hypothesis that stress would moderate the relationships between avoidance and performance on the AMT was not supported. In regression analyses, interactions between avoidance measures and stress predicted no significant variance in the proportion of AMT general or specific responses. This was unexpected given the literature indicating that people higher on avoidance might exhibit overgeneral memory only when under stress (e.g., Debeer et al., 2011).

The third hypothesis that events remembered to positive and negative cues would be rated as pleasant and unpleasant, respectively was supported. Pleasantness ratings to positive and negative cues were in the upper and lower halves of the rating scale, respectively, and were significantly different from the midpoint of the scale. In addition, there was a significant difference between pleasantness ratings of responses to positive and negative cues.

The fourth prediction, that higher self-reported avoidance would be associated with a lower proportion of categoric AMT-R responses, was made tentatively. Also in an exploratory 
vein, I examined the relationship between avoidance and proportion of AMT-R specific responses. There were no significant correlations between AMT-R indices and avoidance measures.

The results with respect to the relationships between AMT indices, avoidance, and stress were unexpected. Therefore, in Study 2, I investigated whether findings from Study 1 could be replicated. 


\section{Chapter 3}

\section{Study 2: Replication of AMT Findings from Study 1}

Some wording in this chapter is verbatim from Ganly et al. (2016).

\section{Introduction}

Because, in Study 1, the direction of the relationship between proportion of AMT overgeneral responses and CBAS total and CBAS-C scores was at odds with some previous research (Hermans et al., 2005), and there were no significant correlations or notable effect sizes between avoidance measures and AMT-R indices, the research focus of the thesis was narrowed in Study 2. In particular, I narrowed the focus of the overall research project to the relationship between key variables and AMT indices only, as opposed to also examining AMT-R indices. With this in mind, in Study 2, the AMT-R was not administered.

Study 2 research aims. Study 2 tested hypotheses 1, 2, and 3 as they were outlined in Study 1. Study1's fourth hypothesis pertained to the AMT-R and was therefore omitted.

\section{Method}

Participants. I wished to replicate Study 1 with greater power and I therefore recruited 133 participants. They were selected using the same criteria as Study 1 and were awarded course credit for participation. Data from three participants were removed from analyses due to large amounts of missing data. Of the remaining 130 participants, 105 (81\%) were female. Of the 128 who reported their age, the mean age was 18.31 years $(S D=0.54)$.

Materials. As in Study 1, participants were administered the AMT, CBAS, AAQ-II, WBSI, and DASS. The AMT was administered with cue-set 1 . AMT responses from $25 \%$ of participants, randomly selected, were coded by an independent rater and Kappa was acceptably high, $K=.76$. Disagreements were resolved by the primary rater (TG). I also administered the reflection and brooding subscales from the Ruminative Responses Scale (Treynor et al., 2003); Marlowe Crowne Social Desirability Scale (Crowne \& Marlowe, 1960); Manifest Anxiety Scale (Taylor, 1953); and Repetitive Thinking Questionnaire (McEvoy, Thibodeau, \& Asmundson, 2014). As they are not relevant to the aims of the current studies, they are not reported further.

Procedure. Participants were administered the AMT, then the measures of avoidance (CBAS, AAQ-II, and WBSI), then the DASS. Participants carried out the procedure in groups of 2-10 participants. The procedure for consent and debriefing was identical to Study 1. The School 
of Psychology Human Ethics Committee under the delegated authority of Victoria University of Wellington's Human Ethics Committee approved this study.

\section{Results}

Missing data. There was one missing pleasantness rating for autobiographical memories. There was one item missing in each of the DASS, and AAQ-II, and five in the CBAS. These were imputed using the same methods described in Study 1.

Descriptive statistics. Study 2 variables are shown in Table 3.1. DASS depression and stress were in the mild range, slightly elevated compared to those in Study 1.

\section{Hypothesis 1: Relationship between avoidance and AMT indices. Correlations}

between study variables are shown in Table 3.2. As can be seen, there was a significant negative correlation between CBAS total scores and proportion of overgeneral responses, replicating the finding from Study 1. In addition, however, there was a significant positive correlation between CBAS total scores and the proportion of specific responses. When the CBAS subscales were examined, the CBAS-C subscale was significantly negatively correlated with proportion of overgeneral AMT responses. The correlations between WBSI scores and AMT indices evidenced the same pattern as the CBAS total score and AMT indices. There were no statistically significant relationships between AAQ-II scores and AMT indices. These results offer mixed support for the hypothesis that there would be no correlation between avoidance measures and AMT indices. 
Table 3.1

Descriptive Statistics for Study 2 Variables

\begin{tabular}{lcrcr}
\hline \multicolumn{1}{c}{ Variable } & $M$ & $S D$ & Minimum & Maximum \\
\hline AMT proportion spec & .83 & .20 & .00 & 1.00 \\
AMT proportion OG & .10 & .10 & .00 & .50 \\
AMT pleasantness pos & 4.16 & 0.52 & 2.60 & 5.00 \\
AMT pleasantness neg & 1.67 & 0.43 & 1.00 & 3.00 \\
DASS depression & 11.56 Mild & 10.21 & 0.00 & 42.00 \\
DASS stress & 15.09 Mild & 9.81 & 0.00 & 40.00 \\
AAQ-II & 23.71 & 9.56 & 8.00 & 46.00 \\
CBAS total & 66.49 & 18.57 & 37.00 & 124.00 \\
CBAS-B & 28.86 & 9.13 & 15.00 & 66.00 \\
CBAS-C & 37.63 & 11.67 & 18.00 & 81.00 \\
WBSI & 53.45 & 11.72 & 18.00 & 75.00 \\
\hline
\end{tabular}

Note. AMT indices are proportions excluding omissions. AAQ-II = Acceptance and Action Questionnaire II; AMT = Autobiographical Memory Test; CBAS = Cognitive Behavioral Avoidance Scale; CBAS-B = CBAS behavioural subscale; CBAS-C = CBAS cognitive subscale; DASS = Depression Anxiety Stress Scales 21-item version; Mild = DASS severity label; Neg = negative; $\mathrm{OG}=$ overgeneral responses; $\mathrm{Pos}=$ positive; $\mathrm{Spec}=$ specific memories; $\mathrm{WBSI}=$ White Bear Suppression Inventory. 
Table 3.2

Correlations [and 95\% BCa Confidence Intervals] between Depression, Stress, Avoidance, and AMT Indices in Study 2

\begin{tabular}{|c|c|c|}
\hline Variable & AMT spec & AMT OG \\
\hline \multirow[t]{2}{*}{ DASS depression } & .16 & -.14 \\
\hline & {$[.027, .284]$} & {$[-.287,-.001]$} \\
\hline \multirow[t]{2}{*}{ DASS stress } & .09 & -.07 \\
\hline & {$[-.039, .205]$} & {$[-.217, .081]$} \\
\hline \multirow[t]{2}{*}{ AAQII } & .14 & -.11 \\
\hline & {$[-.011, .293]$} & {$[-.260, .034]$} \\
\hline \multirow[t]{2}{*}{ CBAS total } & $.18^{*}$ & $-.20 *$ \\
\hline & {$[.029, .326]$} & {$[-.329,-.064]$} \\
\hline \multirow[t]{2}{*}{ CBAS-B } & .15 & -.16 \\
\hline & {$[.025, .263]$} & {$[-.287,-.017]$} \\
\hline \multirow[t]{2}{*}{ CBAS-C } & .17 & $-.19 *$ \\
\hline & {$[-.018, .343]$} & {$[-.358,-.024]$} \\
\hline \multirow[t]{2}{*}{ WBSI } & $.19 *$ & $-.28 * *$ \\
\hline & {$[.030, .358]$} & {$[-.445,-.115]$} \\
\hline
\end{tabular}

Note. AMT indices are proportions excluding omissions. AAQII $=$ Acceptance and Action Questionnaire II; AMT = Autobiographical Memory Test; CBAS = Cognitive Behavioral Avoidance Scale; CBAS-B = CBAS behavioural subscale; CBAS-C = CBAS cognitive subscale; DASS = Depression Anxiety Stress Scales 21-item version; OG = Proportion of overgeneral memories; Spec $=$ Proportion of specific memories; WBSI $=$ White Bear Suppression Inventory. $\mathrm{s}^{*} p<.05 . * * p<.01$.

\section{Hypothesis 2: Stress as a moderator of the relationship between avoidance and}

AMT indices. The moderating role of stress in the relationship between avoidance and AMT indices was examined using the same approach as in Study 1. When predicting proportion of 
specific responses, the third steps of these models were not significant, $F \mathrm{~s}=1.09$ to $1.61, p \mathrm{~s}=$ .190 to .355 . When predicting proportion of overgeneral responses, the third steps of the models involving AAQ-II, CBAS total, CBAS-B, and CBAS-C were not significant, $F \mathrm{~s}=0.57$ to 1.63 , $p s=.155$ to .638 . The third step of the model involving the WBSI score was significant, $R^{2}=$ .08 , adjusted $R^{2}=.07, F(3,126)=3.71, p=.013$, but in this model, the WBSI $\times$ stress interaction term was not significant, $B=0.000007, \beta=-.04, t(126)=.09, p=.927, \mathrm{BCa} 95 \% \mathrm{CI}$ $[-0.0001,0.0002]$. These results did not support the hypothesis that stress would moderate the relationship between avoidance and AMT indices.

\section{Hypothesis 3: Pleasantness ratings of autobiographical memories. Pleasantness} ratings to positive cues were higher than the pleasantness rating scale's midpoint (3), a statistically significant mean difference of 1.16 , BCa 95\% CI $[1.07,1.25], t(129)=25.65, p<$ .001. Pleasantness ratings to negative cues were lower than the midpoint, a statistically significant mean difference of -1.33 . BCa 95\% CI [-1.41, -1.25], $t(129)=-35.16, p<.001$.

The difference in mean pleasantness ratings for memories reported to positive and negative AMT cues was 2.49 , BCa 95\% CI [2.36, 2.61]. This difference was significant, $t(129)=$ $37.68, p<.001$. In line with Study 1 findings, these results indicated that positive and negative cues were effective in eliciting memories for pleasant and unpleasant events, respectively. Further examination of these pleasantness rating data are presented in Chapter 8.

\section{Discussion}

There was a negative relationship between avoidant coping (on CBAS, CBAS-C, and WBSI scores) and proportion of overgeneral responses on the AMT. In addition, there was a positive correlation between CBAS total and WBSI scores and proportion of AMT specific responses. That is, individuals who scored higher on these two measures of avoidance also reported a greater proportion of specific responses on the AMT relative to lower scoring participants. These findings from Studies 1 and 2 do not support the idea that that remembering less specifically may form part of an avoidant coping style (Hermans et al., 2005). In the present studies, avoidant coping was associated with a higher proportion of AMT specific responses (Study 2) and a lower proportion of AMT overgeneral responses (Studies 1 and 2). Furthermore, stress did not play a role in the relationship between avoidant coping and AMT performance in either Study 1 or Study 2, at odds with Debeer et al.'s (Debeer et al., 2012, 2011, 2013) findings that people higher on avoidant coping experienced decreases in memory specificity while under 
stress.

A possible reason for this finding is that a different pattern of relationships between avoidance and memory specificity might be found for individuals experiencing higher levels of depression. The possibility that the relationship between overgeneral memory and the mechanisms of the CaR-FA-X model may differ between different populations was raised by Sumner (2012). Further support for this possibility is found in longitudinal research with youth, where significant relationships between specific and overgeneral memory and psychological functioning were found only for the group of young people already experiencing high levels of psychological distress (Gutenbrunner, Salmon, \& Jose, 2017a). Following recommendations by Sumner, that CaR-FA-X mechanisms should be investigated separately in different samples (e.g, currently depressed vs. healthy individuals), I carried out a third study, replicating Study 2's method in a sample experiencing depressed mood. 


\section{Chapter 4}

\section{Study 3: The Relationships between Avoidance, Overgeneral Memory, and Stress in a Clinical Sample}

Some wording in this chapter is verbatim from Ganly et al. (2016).

\section{Introduction}

Study 3 addressed Research Aim 1c of this thesis, to investigate the relationship between AMT indices, avoidant coping, and stress in a clinical sample (cf., non-clinical, undergraduate samples used in Studies 1 and 2). To this end, I investigated the relationship between avoidance and AMT indices in a sample currently experiencing depressed mood recruited from a counselling service. Specifically, I predicted that participants high on avoidance would report a lower proportion of specific AMT responses and higher proportion of general AMT responses as compared to those low on avoidance (hypothesis 1). I also predicted that stress would moderate the relationship between avoidance and AMT indices such that high avoiders self-reporting high levels of stress would manifest greater overgeneral memory than those not self-reporting high levels of stress (hypothesis 2). Based on Research Aim 3 of this thesis, I also hypothesised that positive and negative AMT cues would elicit memories for positive and negative events, respectively (hypothesis 3).

\section{Method}

Participants. Participants were 52 students recruited from the Student Counselling Service at Victoria University of Wellington, New Zealand. The mean age was 24.04 years ( $S D$ $=7.65)$, and $42(81 \%)$ were female. I reasoned that levels of psychological distress would be higher than in the general population in this group, and therefore, in 2015, an email was sent to everyone who had accessed the service in 2014 asking them if they would like to participate in a study that aimed "to investigate the way people remember events from their past and how this is related to depression". The email stated that to be eligible, participants should "currently be experiencing depressed mood" and that their first language should be English. A movie voucher was offered for participation. Potential participants who emailed the primary researcher were then asked to confirm they met the selection criteria. If the criteria were met, they were scheduled to carry out the procedure. Sample size was therefore determined by the number of respondents who met criteria. DASS depression scores in this group (see Table 4.1) were comparable to those reported in a sample of in- and outpatients diagnosed with a depressive 
disorder (Page, Hooke, \& Morrison, 2007). This indicated that I was successful in recruiting a depressed sample.

Materials and procedure. Materials were as in Study 2. AMT responses from $25 \%$ of participants, randomly selected, were coded by an independent rater and Kappa was acceptably high, $K=.78$. Participants carried out the procedure individually. Participants signed a written consent form. On completion of the procedure, all participants received a written debriefing statement, any questions participants had about the study were answered, and participants were given a movie voucher. The study was approved by the School of Psychology Human Ethics Committee under the delegated authority of Victoria University of Wellington's Human Ethics Committee.

\section{Results}

Missing data. There was one missing item in the WBSI, two in the CBAS, and three in the DASS. These were imputed using same method as Studies 1 and 2.

Descriptive statistics. Descriptive statistics are shown in Table 4.1. Depression and stress scores were in the severe to moderate range for DASS scores (Lovibond \& Lovibond, 1995). A MANOVA with sample (Study 2 vs. Study 3) as the independent variable and DASS depression, DASS stress, AAQ-II, CBAS total, and WBSI, scores as dependent variables established that levels of psychopathology differed between studies. There was a significant effect of sample on the combined dependent variables, $F(5,176)=16.54, p<.001$, partial $\eta^{2}=$ .32. Analyses of the dependent variables individually indicated that DASS depression and stress, AAQ-II, CBAS total, and WBSI scores were all significantly higher in Study 3 than in Study 2, all $p s<.001$. A separate MANOVA examined the difference in CBAS subscales (CBAS-B and CBAS-C) between Studies 2 and 3. This MANOVA was also significant, $F(2,179)=44.10, p<$ .001 , partial $\eta 2=.33$. Analysing the subscales individually showed that both subscale scores were significantly higher in Study 3 compared to Study 2, both $p$ s $<.001$. 
Table 4.1

Descriptive Statistics for Study 3 Variables

\begin{tabular}{lrrrr}
\hline \multicolumn{1}{c}{ Variable } & \multicolumn{1}{c}{$M$} & SD & Minimum & Maximum \\
\hline AMT proportion spec & .86 & .11 & .30 & 1.00 \\
AMT proportion OG & .08 & .11 & .00 & .40 \\
AMT pleasantness pos & 3.81 & 0.61 & 2.25 & 4.80 \\
AMT pleasantness neg & 1.48 & 0.37 & 1.00 & 2.60 \\
DASS depression & 22.22 Severe & 11.23 & 4.00 & 40.00 \\
DASS stress & 23.00 Moderate & 8.14 & 8.00 & 40.00 \\
AAQ-II & 36.81 & 7.63 & 17.00 & 49.00 \\
CBAS total & 87.62 & 21.41 & 45.00 & 132.00 \\
CBAS-B & 42.98 & 10.27 & 20.00 & 66.00 \\
CBAS-C & 44.64 & 13.45 & 44.64 & 73.00 \\
WBSI & 61.58 & 7.07 & 49.00 & 74.00 \\
\hline
\end{tabular}

Note. AMT indices are proportions excluding omissions. AAQ-II = Acceptance and Action

Questionnaire II; AMT = Autobiographical Memory Test; AMT-R = Autobiographical Memory Test - Reversed; CBAS $=$ Cognitive Behavioral Avoidance Scale; CBAS-B = CBAS

behavioural subscale; CBAS-C = CBAS cognitive subscale; DASS = Depression Anxiety Stress Scale 21-item version; Moderate $=$ DASS severity label; Neg = negative; $\mathrm{OG}=$ overgeneral responses; Pos = positive; Severe $=$ DASS severity label; Spec $=$ specific responses; WBSI = White Bear Suppression Inventory.

Hypothesis 1. Relationship between avoidance and AMT indices. As can be seen in Table 4.2, there were no significant correlations between AMT indices and measures of avoidant coping or stress. Nonetheless, it is important to note the size of the correlation between proportion of specific memories and AAQ-II scores was similar to that found in Study 2 for correlations between proportion of specific memories and AAQ-II (though non-significant), CBAS, and WBSI scores, but in the opposite (expected) direction, $r(50)=-.19, p=.183$.

\section{Hypothesis 2. Stress as a moderator of the relationship between avoidance and AMT} indices. The moderating role of stress in the relationship between avoidance and AMT indices was examined using the same approach as Studies 1 and 2. When predicting proportion of 
specific responses, the third steps of these models were not significant, $F \mathrm{~s}=0.57$ to $1.86, p \mathrm{~s}=$ .149 to .639 . When predicting proportion of overgeneral responses, the third steps of the models involving AAQ-II, CBAS total, CBAS-B, and WBSI were not significant, $F \mathrm{~s}=1.26$ to 2.27, $p \mathrm{~s}=$ .092 to .299 . The third step of the model involving the CBAS-C was significant, $R^{2}=.16$, adjusted $R^{2}=.11, F(3,48)=3.12, p=.035$, but in this model, the CBAS-C $\times$ stress interaction term was not significant, $B=-0.0003, \beta=1.42, t(77)=-2.00, p=.051$, BCa 95\% CI [-0.0005, 0.00003]. These results offered no support for the hypothesis that stress would moderate the relationship between AMT indices and avoidance.

Hypothesis 3. Pleasantness ratings of autobiographical memories. Pleasantness ratings to positive cues were higher than the pleasantness rating scale's midpoint (3), a statistically significantly mean difference of 0.81 , BCa 95\% CI $[0.63,0.96], t(51)=9.53, p<$ .001. Pleasantness ratings to negative cues were lower than the midpoint, a statistically significant mean difference of -1.52 , BCa 95\% CI [-1.62, -1.42], $t(51)=-29.38, p<.001$.

The difference in mean pleasantness ratings between responses to positive and negative AMT cues was 2.33, BCa 95\% CI [2.14, 2.51]. As was the case in Studies 1 and 2, this difference was significant, $t(51)=23.82, p<.001$. As found in Studies 1 and 2, these results supported the hypothesis that positive and negative cues would elicit memories for pleasant and unpleasant events, respectively. Further examination of these pleasantness rating data are presented in Chapter 8. 
Table 4.2

Correlations [and 95\% BCa Confidence Intervals] between Depression, Stress, Avoidance, and AMT Indices in Study 3

\begin{tabular}{lcc}
\hline \multicolumn{1}{c}{ Variable } & AMT spec & AMT OG \\
\hline DASS depression & .08 & .01 \\
& {$[-.168, .335]$} & {$[-.215, .244]$} \\
DASS stress & .15 & -.22 \\
& {$[-.142, .463]$} & {$[-.394,-.036]$} \\
AAQII & -.19 & .01 \\
& {$[-.418, .097]$} & {$[-.211, .227]$} \\
CBAS total & -.05 & .05 \\
& {$[-.295, .229]$} & {$[-.189, .285]$} \\
CBAS-B & -.02 & -.09 \\
& {$[-.274, .268]$} & {$[-.314, .138]$} \\
CBAS-C & -.05 & .15 \\
WBSI & {$[-.334, .229]$} & {$[-.134, .426]$} \\
& -.07 & -.06 \\
& $-341, .258]$ & {$[-.372, .253]$} \\
& &
\end{tabular}

$\overline{\text { Note. } \text { AMT indices are proportions excluding omissions. AAQII }}=$ Acceptance and Action Questionnaire II; AMT = Autobiographical Memory Test; CBAS = Cognitive Behavioral Avoidance Scale; CBAS-B = CBAS behavioural subscale; CBAS-C = CBAS cognitive subscale; DASS $=$ Depression Anxiety Stress Scales 21-item version; OG = Proportion of overgeneral responses; Spec $=$ Proportion of specific responses; WBSI $=$ White Bear Suppression Inventory. 


\section{Chapter 5}

\section{Interim Summary}

This Chapter has extremely similar wording to the General Discussion in Ganly et al. (2016). I kept most of the wording from the published article to preserve the link between the thesis and published article.

Study 3 was conducted to understand unexpected findings from Studies 1 and 2: Avoidant coping was associated with a higher proportion of specific responses (Study 2) and a lower proportion of overgeneral responses (Studies 1 and 2) on the AMT. Stress did not moderate these relationships. Levels of depression were low in these samples, however, and I reasoned that in a sample with higher levels, the expected relationships (avoidant coping associated with lower proportion of specific memories and higher proportion of overgeneral memories) may be found. Therefore, study 3 participants were recruited from a student counselling service and had significantly higher levels of psychological distress than Study 2 participants. In this sample, however, there were no significant correlations between measures of avoidant coping and AMT indices. Note, however, that the (non-significant) negative correlation between AAQII scores and specific memories shown in Table 4.2 was in the predicted direction.

Study 1 and 2's results show, to my knowledge, an as yet unobserved pattern of relationships between avoidant coping and AMT performance. Whereas Hermans et al. (2005) found a negative correlation between avoidant coping and memory specificity, Debeer et al. (Debeer et al., 2012, 2011) reported a more nuanced relationship such that high avoiders' specificity reduced compared to low avoiders, but only when experiencing stress. The current studies' results are different again: the opposite pattern to that observed by Hermans et al., and with no moderating role of stress. An underlying assumption of much research in this area is that remembering less specifically may be utilised to some degree by psychologically healthy individuals without necessarily being an indicator of psychopathology and that it may be activated in times of stress (Debeer et al., 2011, 2012; Hermans et al.). I did not find support for this view.

I speculated that the pattern of relationships observed with the AMT may be normative in a sample with relatively low overall depression scores. Recall that in the Study 1 and 2 samples, mean DASS depression scores were in the normal to mild range. In Study 3, therefore, I recruited a sample experiencing higher levels of depression than in Studies 1 and 2. Although 
there were no significant correlations between avoidant coping measures and AMT indices in Study 3, the correlation between experiential avoidance and AMT-specific responses was in the direction (negative) predicted by the functional avoidance view.

Why was the pattern of AMT results in Studies 1 and 2 at odds with previous research and theory? One possible explanation for this occurring in psychologically healthy people (Studies 1 and 2) is forwarded by Phung and Bryant (2013). They argued that attempting to avoid specific memories may actually result in remembering more specific memories, opposite to predictions derived from the CaR-FA-X model's functional avoidance mechanism. They based this idea on ironic process theory (Wenzlaff \& Wegner, 2000) which posits that two processes occur when a person attempts to suppress a thought. The first is the operating process, an effortful process that searches for alternative distracting thoughts. The second is the monitoring process, a less effortful process that simultaneously checks the to-be-suppressed thought is not present in consciousness. The monitoring process re-engages the operating process if traces of the to-be-suppressed thought are encountered so that suppression can be maintained. When the operating process is voluntarily terminated, the monitoring process may remain vigilant, increasing sensitivity to the target thought, and hence increase the likelihood that it will occur.

When applied to autobiographical memory, Phung and Bryant (2013) argued that an opposite prediction to the CaR-FA-X model can be derived: functional avoidance amounts to thought suppression, and should therefore be associated with more specific memories and fewer overgeneral memories because attempts to suppress specific memories fail, increasing their occurrence. This is consistent with the idea that suppression may enhance memory for suppressed information (Wenzlaff \& Wegner, 2000), and that intrusive memories tend to be specific rather than categoric (Reynolds \& Brewin, 1998). It may be, therefore, that functional avoidance (in so much as it involves attempts to actively suppress specific memories) increases the accessibility of specific memories, at least in non-clinical individuals. Phung and Bryant found some support for this in an experiment with undergraduate students; participants who were actively engaged in suppressing thoughts about a distressing video retrieved fewer categoric memories than those not suppressing thoughts. This is consistent with Study 1 and 2 results and indicates that functional avoidance (as evidenced by an avoidant coping style in the current study) may not be the best account of reduced memory specificity in non-clinical populations. 
Studies 1 and 2 and Phung and Bryant's (2013) work did not focus on clinical samples, however, and as the CaR-FA-X model is an account of the relationship between memory specificity and psychopathology, I recruited Study 3 participants who were currently experiencing depressed mood. Although there were no significant relationships between avoidant coping measures and AMT indices in Study 3, it is interesting to note that the Pearson's correlation between AAQ-II scores and proportion of specific memories in Study 3 was negative $(r=-.19)$. This is to be contrasted with the (non-significant) positive correlation in Study $2(r=$ .14). Further to this, it is important to note that not all previous research has found the expected associations between memory specificity and depression within non-clinical groups. For example, Raes, Hermans, Williams, and Eelen (2007) discussed two unpublished studies in which depressive symptoms in non-clinical groups of students were associated with memory specificity in unexpected ways. In the first, there was no association. In the second, depressive symptoms were associated with higher memory specificity and lower overgenerality. In a similar vein, Smets, Griffith, Wessel, Walschaerts, and Raes (2013) reported that trait rumination did not predict changes in overgeneral memory following a state-rumination induction.

I suggest that in psychologically healthy people, avoidant coping is associated with increases in memory specificity perhaps due to failures in thought suppression (our Studies 1 and 2; Phung \& Bryant, 2013). For individuals experiencing psychopathology, however, avoidant coping is associated with reductions in memory specificity (Study 3), that is, the relationship "switches", changes direction. This switch may occur because as psychological distress increases, so too do avoidant cognitions and behaviours (Ottenbreit \& Dobson, 2004), and painful specific memories begin to have serious adverse implications for psychological wellbeing leading to other components of the CaR-FA-X model (capture and rumination, executive control difficulties) taking effect. I make this suggestion tentatively, because the corresponding confidence intervals for correlations in each study (e.g. for the correlation between CBAS scores and specific responses for Studies 2 and 3) overlap to a large degree, indicating the absence of significant differences between them.

Finally, I point out several limitations to these studies. First, because the design was correlational, causal inferences about the relationships between avoidant coping and memory specificity are difficult. Second, as current levels of depressive symptomology were measured using self-report (DASS depression scores) rather than clinical diagnosis of depression, our 
findings may underestimate the relationship between overgeneral memory and avoidance in clinical populations. Specifically, it may be that overgeneral memory is especially associated with diagnosis of depressive disorder rather than depressive symptomology in subclinical groups (Williams et al., 2007), that is, it is a matter of degree. Nonetheless, I note that Study 3 participants' self-reported level of depression was in the "severe" range, what might be expected in a diagnosed group (Page et al., 2007). Third, I administered the AMT in written (rather than oral) form and with traditional (rather than minimal) instructions. This approach was taken in order to replicate Hermans et al.'s (2005) methodology. I acknowledge the need to replicate our findings using other modes of AMT administration, however, because as Griffith et al. (2012) point out, methodological variation in AMT administration may limit comparisons I can make between studies. Fourth, I also acknowledge that although the findings for the relationship between overgeneral memories and CBAS scores are consistent between Studies 1 and 2, the findings for specific memories are not. As suggested by Debeer et al. (2012), inconsistency in findings for specific and overgeneral responses could be related to a lack of power. By increasing the sample size in Study 2, the effect for the relationship between CBAS scores and specific responses was observed. In a similar vein, the sample size for Study 3 was rather low, limiting conclusions that can be made.

As far as I am aware, this is the first study to examine the relationship between avoidance and memory specificity and overgenerality in nondepressed and depressed samples. The unexpected AMT results highlight the need for more research in this area and suggest that a more nuanced relationship between avoidance and memory specific than is presented in the CaR-FA-X model may exist. 


\section{Chapter 6}

\section{Study 4: The Influence of Rating Event Pleasantness on Overgeneral Memory}

\section{Introduction}

The motivation behind Study 3 was to attempt to explain the findings from Study 2. Specifically, the research question was: Why, in a non-clinical sample, was avoidance positively correlated with proportion of overgeneral responses, and negatively correlated with proportion of specific AMT responses? The previous chapter investigated the idea that the correlation predicted by the affect-regulation hypothesis (Williams, 1996) would emerge in a sample experiencing higher levels of depression. Indeed, mean depression levels in Studies 1 and 2 were in the normal to mild range for DASS depression scores. In this Chapter, I test another explanation for Study 2's findings: the idea that asking participants to rate the (un)pleasantness of remembered events on the AMT influenced those who were higher on avoidance to remember more specific and fewer overgeneral memories than they would under administration of the AMT with no pleasantness rating scale. In this section, I present a justification for this idea.

AMT administration in Studies 1, 2, and 3 differed from most previous research (but see Geraerts et al., 2012) by the addition of the pleasantness rating scale on each page of the response booklet and the associated instructions that were given to participants about how to complete the scale. As described in Chapter 2, participants were told that for each memory, they should rate the pleasantness of the remembered event on a 5-point Likert scale printed at the bottom of each page with anchors: $1=$ "Extremely unpleasant" and $5=$ "Extremely pleasant". Explicit instructions about how to complete the rating (think back to the event and decide how pleasant or unpleasant it was, then circle the appropriate number) were presented to participants before the AMT began. An unintended effect of presenting explicit instructions to consider the (un)pleasantness of remembered events and presenting the printed pleasantness rating scale on every page could have been to greatly emphasise to participants that the pleasantness or unpleasantness of remembered events was of key importance to the studies' aims. This may have increased the degree to which participants were reflecting on the pleasant or unpleasant emotional aspects of events during retrieval.

The possibility that the pleasantness rating scale on each page of the response booklet served as a cue is worth considering. Conway and Pleydell-Pearce (2000) emphasised the cued nature of remembering within the SMS. In the SMS, a cue is any mnemonic unit at any level of 
abstraction, and the SMS's autobiographical knowledge base is "exquisitely sensitive to cues" (Conway \& Loveday, 2010, p. 57). In Studies 1, 2, and 3, the presence of the words "Extremely pleasant" and "Extremely unpleasant" on each page of the booklet in highly salient fashion could have inadvertently served as additional cues during each AMT trial. If this was the case, then the notion of extreme (un)pleasantness as well as the particular emotional cue word would have formed the basis for the elaboration phase of generative retrieval for each trial. In that sense, the pleasantness rating scale and cue word together could be considered a sort of compound cue. As discussed in Chapter 1, the elaboration phase of generative retrieval involves making the cue relevant to the self, and, as Williams (1999) stated, is equivalent to answering the question implied in the cue. Under typical administration of the AMT, elaborating the cue lonely, for example, might have been equivalent to answering the question, "What event made me lonely?" Because of the pleasantness rating scale, in the current studies, elaborating the cue "lonely" might have been equivalent to answering the question, "What extremely pleasant or unpleasant event made me lonely?" In this respect, AMT administration in Studies 1, 2, and 3, differed from its normal method of administration in such a way as to potentially influence the way cues were elaborated by participants.

In summary, the increased focus on the emotional (un)pleasantness of remembered events and its possible influence on cue-elaboration during the administration of the AMT could have led to a way of remembering that differed from normal AMT administration.

\section{Why would rating pleasantness cause avoidant participants to remember more} specifically? Given the unexpected relationships between avoidance and memory specificity/overgenerality found in the current Studies and the methodological difference between the current studies (presence of the pleasantness rating scale) and most previous research (no pleasantness rating scale), a worthwhile question is: Could the increased focus on the emotional aspects of events have increased the specificity of some participants' responses, in particular, those participants who were higher on avoidance? If the answer to this question is yes, then it would explain Study 2's findings. A reasonable follow-up question would be to ask: What is the psychological mechanism by which focusing on the (un)pleasantness of remembered events on the AMT would lead a more highly avoidant person to be more specific and less overgeneral? One possible answer to this question is that more highly avoidant participants (i.e., 
those participants who may have wished to keep emotional aspects of memories at bay), may have experienced an enhancement effect of thought suppression (Wenzlaff \& Wegner, 2000).

The enhancement effect of thought suppression occurs when a person attempting to suppress a thought experiences the increased occurrence of that thought (Wenzlaff \& Wegner, 2000). As discussed in Chapter 5, ironic process theory posits the existence of two processes that occur simultaneously when attempting to suppress a target thought. The operating process is the effortful search for distractions. The monitoring process is the less effortful, continual checking of consciousness for the target thought. If the monitoring process encounters the target thought, it re-engages the operating process. In Chapter 5, I discussed Phung and Bryant's (2013) argument that ironic process theory can be applied to the specificity of autobiographical memory; in short, they argued that suppressing specific memories may increase their frequency, an opposite prediction to the affect-regulation hypothesis. In a further elaboration on Phung and Bryant's idea, I will now discuss how ironic process theory can be used to address the question: What is the possible psychological mechanism that may have led more avoidant people to give higher and lower proportions of specific and overgeneral AMT responses, respectively?

Consider the following sequence of events that may have unfolded during AMT administration in Study 2: Participants were exposed to the task instructions regarding remembering personally experienced events and making judgements about the (un)pleasantness of those events. For participants higher on avoidance, this invoked an alertness to the possibility of remembering unpleasant memories. That is, those people who prefer not to think about the (un)pleasantness of the things they remember, may have begun to prepare themselves for doing just that. This mental preparation amounted to the initiation of the operating and monitoring processes as described in ironic process theory.

As the AMT starts, avoidant participants are asked to begin remembering events, and, as a consequence, they have to contend with memories they may prefer not to encounter, increasing their attempts at thought suppression. Because of the time pressure (one minute per cue), the emotional nature of the cue words, the instruction to rate the pleasantness of events, and the necessity to keep other task instructions in mind, the cognitive load on high-avoiders could be quite high, and, importantly, relatively higher than low-avoiders. As noted by Wenzlaff and Wegner (2000), attempted thought suppression is most at risk of failure (that is, the target thoughts are most likely to occur) when cognitive load is high. Therefore, attempts at thought 
suppression were more likely to fail; that is, the participants who might ordinarily be adept at curtailing their specific memories instead remembered an uncharacteristically high number of specific memories, and hence, fewer overgeneral memories. In short, the AMT's instructions may have clashed with the avoidant participant's default mode of processing mnemonic information in such a way as to make specific remembering more likely.

In summary, the increased focus on the emotional (un)pleasantness of remembered events during the administration of the AMT may lead to a way of remembering that differed from normal AMT administration. This may have lead participants higher on avoidance to experience an enhancement effect of specific memories, that is, they would be more specific and less overgeneral than they would ordinarily.

Study 4 research aims. In Study 4, I tested the idea that the inclusion of the pleasantness-rating scale on the AMT was associated with an increase in specific responding, but only for those people higher on avoidance, as opposed to those lower on avoidance. In a between-subjects design, participants were randomly allocated to one of two conditions. In the pleasantness rating (PR) condition, participants completed the AMT in the same way as in studies 1,2, and 3, that is, including the pleasantness rating scale. In the no-pleasantness rating (NPR) condition, participants completed the AMT but did not rate the pleasantness of memories, that is, no pleasantness rating scale was included. All participants completed self-report measures of avoidance.

I predicted that condition would interact with avoidance to predict AMT responding. That is, higher avoidance would be associated with a higher proportion of specific memories and lower proportion of general memories but only in the pleasantness rating condition, and not in the no pleasantness rating condition. I also tested hypotheses that were tested in Studies 1, 2, 3 pertaining to the pleasantness ratings of positive and negative cues and the relationships between avoidance, stress, and AMT indices.

\section{Method}

Participants. Participants were 144 undergraduate psychology students recruited in the same way as those in Studies 1 and 2. Participants were awarded course credit for taking part. One participant did not complete the DASS or WBSI and their partial data was not included in any analyses. Of the 143 participants whose data were used, 107 (75\%) were female, and the mean age was $18.40(S D=0.65)$. 
Materials. The AMT was administered the same way as in Studies 1, 2, and 3, except in the no pleasantness rating (NPR) condition $(n=70)$, there were no instructions that they should rate the pleasantness of remembered events. Accordingly, in this condition, the AMT memory booklets did not include the pleasantness rating scale at the bottom of each page. In the pleasantness rating $(\mathrm{PR})$ condition $(n=73)$, instructions for rating pleasantness of remembered events were given to participants as in Studies 1, 2, and 3. Accordingly, the booklets in the PR condition included the pleasantness rating scale at the bottom of each page. All participants were administered the same questionnaire booklet as in Studies 2 and 3 (Cue-set A).

Procedure. The study was approved by the School of Psychology Human Ethics Committee under the delegated authority of Victoria University of Wellington's Human Ethics Committee. The study was carried out in sessions of six to ten participants each. Each session was scheduled to be either the PR or NPR condition; that is, all participants in each session experienced the same condition. In each session, participants were greeted by the researcher and informed, as in Studies 1, 2, and 3 that the study was about how people remember, that their participation was voluntary, and that they were free to withdraw at any time. They were informed that their completion of the questionnaire measures constituted consent.

Participants completed the AMT, either with or without the pleasantness rating according to condition, then completed the questionnaire booklet. After completion of the questionnaires, participants were given a written debriefing statement, and the researcher answered any questions.

\section{Results}

Missing data. There were six missing items in the CBAS, three in the DASS. These were imputed using the same technique as Studies 1, 2, and 3.

Descriptive statistics. Descriptive statistics for the PR condition, NPR condition, and whole sample combined are shown in Table 6.1. 
Table 6.1

Descriptive Statistics for Study 4 Variables in the Whole Sample and by Condition

\begin{tabular}{|c|c|c|c|c|c|c|c|c|c|}
\hline \multirow[b]{2}{*}{ Variable } & \multicolumn{3}{|c|}{ Whole sample } & \multicolumn{3}{|c|}{ PR } & \multicolumn{3}{|c|}{ NPR } \\
\hline & $M(\mathrm{SD})$ & Min & Max & $M(S D)$ & Min & Max & $M(S D)$ & Min & Max \\
\hline AMT prop spec & $.79(.24)$ & .00 & 1.00 & $.81(.22)$ & .00 & 1.00 & $.77(.27)$ & .00 & 1.00 \\
\hline AMT prop OG & $.08(.10)$ & .00 & .43 & $.08(.11)$ & .00 & .43 & $.09(.10)$ & .00 & .38 \\
\hline AMT pleasantness pos & - & - & - & $4.11(0.44)$ & 3.20 & 5.00 & - & - & - \\
\hline AMT pleasantness neg & - & - & - & $1.74(0.43)$ & 1.00 & 2.8 & - & - & - \\
\hline \multirow[t]{2}{*}{ DASS depression } & $14.42(11.11)$ & 0.00 & 40.00 & $14.14(11.37)$ & 0.00 & 40.00 & $14.71(10.90)$ & 0.00 & 40.00 \\
\hline & Moderate & & & Moderate & & & Moderate & & \\
\hline \multirow[t]{2}{*}{ DASS stress } & $16.39(9.43)$ & 0.00 & 38.00 & $16.07(9.48)$ & 0.00 & 36.00 & $16.71(9.44)$ & 2.00 & 38.00 \\
\hline & Mild & & & Mild & & & Mild & & \\
\hline AAQ-II & $26.57(10.36)$ & 7.00 & 47.00 & $26.47(10.23)$ & 7.00 & 45.00 & $26.67(10.56)$ & 8.00 & 47.00 \\
\hline CBAS total & 71.48 (18.24) & 33.00 & 115.00 & $72.44(20.00)$ & 33.00 & 115.00 & $70.46(16.30)$ & 38.00 & 111.00 \\
\hline CBAS-B & $31.82(9.89)$ & 15.00 & 63.00 & $32.16(10.41)$ & 15.00 & 56.80 & $31.47(9.38)$ & 16.00 & 63.0 \\
\hline CBAS-C & $39.65(11.42)$ & 17.00 & 67.00 & $40.29(12.26)$ & 18.00 & 67.00 & $38.99(10.53)$ & 17.00 & 66.00 \\
\hline WBSI & $54.98(12.23)$ & 22.00 & 74.00 & $54.34(12.62)$ & 22.00 & 73.00 & $55.64(11.86)$ & 27.00 & 74.00 \\
\hline
\end{tabular}

Note. AAQ-II = Acceptance and Action Questionnaire II; AMT prop spec = proportion specific AMT responses excluding omissions; AMT prop $\mathrm{OG}=$ proportion overgeneral AMT responses excluding omissions; CBAS = Cognitive Behavioral Avoidance Scale; CBAS-B = CBAS

behavioural subscale; CBAS-C = CBAS cognitive subscale; DASS = Depression Anxiety Stress Scales 21-item version; Max = maximum; Min $=$ minimum $;$ Mild $=$ DASS severity label $;$ Moderate $=$ DASS severity label; Neg = negative NPR = No pleasantness rating condition; Pos $=$ 
positive; $\mathrm{PR}=$ Pleasantness rating condition; $\mathrm{WBSI}=$ White Bear Suppression Inventory. 
Hypothesis tests. The main hypothesis concerned the differences in proportion of specific and overgeneral memories reported by people higher on avoidance under normal administration of the AMT (NPR condition) compared with the modified version used in Studies 1, 2, and 3 (PR condition). If there were statistically significant differences in avoidance between conditions, this could have hindered the interpretation of the main hypothesis test. With that in mind, differences in avoidance measures between conditions were tested using a multivariate analysis of variance (MANOVA) with condition (NPR vs. PR) as the independent variable and AAQ-II, CBAS total, and WBSI, as the dependent variables. The MANOVA was statistically non-significant, $F(3,139)=.441 ., p=.724$. A second MANOVA with condition as the independent variable and the CBAS subscales (CBAS-B and CBAS-C) as dependent variables was also non-significant, $F(2,140)=.234, p$ $=.791$. These results indicated there were no significant differences in avoidance measures between conditions.

I hypothesised that asking participants to rate the (un)pleasantness of events influenced those who were higher on avoidance to remember in a more specific way. That is, participants asked to rate the pleasantness of remembered events (PR condition) would be more specific in the way they remembered than participants not asked to rate their pleasantness (NPR condition), but that this effect would only be present for those high on avoidance. To test this interaction between condition and avoidance, a series of hierarchical multiple regressions were conducted with either proportion of specific AMT responses or proportion of overgeneral AMT responses as the outcome variable. Condition was dummycoded, $\mathrm{PR}=0, \mathrm{NPR}=1$. At the first step of each regression, the particular avoidance variable score was entered (i.e., AAQ-II, CBAS total, CBAS-B, CBAS-C, or WBSI). At the second step, condition was entered. At the third step, the interaction term (e.g., AAQ-II $\times$ condition) was entered. When predicting proportion of specific responses, the third steps of these models were not significant, $F \mathrm{~s}=0.82$ to $2.61, p \mathrm{~s}=.054$ to .485 . When predicting proportion of overgeneral responses, the third steps of these models were not significant, $F \mathrm{~s}=0.14$ to $0.72, p s=.541$ to .934 . These results did not support the hypothesis that asking participants to rate the (un)pleasantness of events would influence those who were higher on avoidance to remember in a more specific way. 
Table 6.2

Correlations [and 95\% BCa Confidence Intervals] between Depression, Stress, Avoidance, and AMT Indices in Study 4 (NP and NPR Condition Combined)

\begin{tabular}{lcc}
\hline \multicolumn{1}{c}{ Variable } & AMT spec & AMT OG \\
\hline DASS depression & .09 & -.02 \\
& {$[-.081, .238]$} & {$[-.168, .143]$} \\
DASS stress & .05 & .03 \\
& {$[-.122, .212]$} & {$[-.120, .197]$} \\
AAQII & -.03 & -.01 \\
& {$[-.218, .164]$} & {$[-.170, .164]$} \\
CBAS total & -.12 & -.03 \\
& {$[-.251, .002]$} & {$[-.194, .143]$} \\
CBAS-B & -.11 & -.01 \\
& {$[-.280, .049]$} & {$[-.171, .154]$} \\
CBAS-C & -.10 & -.04 \\
WBSI & {$[-.224, .029]$} & {$[-.203, .132]$} \\
& -.03 & .07 \\
& {$[-.240, .178]$} & {$[-.105, .242]$}
\end{tabular}

$\overline{\text { Note. } \text { AMT indices are proportions excluding omissions. AAQII }}=$ Acceptance and Action Questionnaire II; AMT = Autobiographical Memory Test; CBAS = Cognitive Behavioral Avoidance Scale; CBAS-B = CBAS behavioural subscale; CBAS-C = CBAS cognitive subscale; DASS = Depression Anxiety Stress Scales 21-item version; OG = Proportion of overgeneral responses; Spec $=$ Proportion of specific responses; WBSI $=$ White Bear Suppression Inventory.

$* p<.05 . * * p<.01$.

Relationships between stress, avoidance, and AMT performance. Because there was no interaction between condition and avoidance measures, data for both conditions were combined. In this combined sample, the relationships between stress, avoidance, and AMT performance were examined as per Studies 1, 2, and 3. First, bivariate relationships between avoidance, stress, and AMT performance were examined. As can be seen in seen in Table 
6.2, all of the CIs include 0 ; moreover, none of these relationships were statistically significant.

To test the interactions between stress and avoidance measures in predicting AMT performance, the same approach was taken as in Studies 1, 2, and 3. When predicting proportion of specific responses, the third steps of the models involving AAQ-II, CBAS-C, and WBSI were not significant, $F \mathrm{~s}=0.48$ to $2.41, p \mathrm{~s}=.070$ to .695 . The third step of the model involving CBAS total was significant, $R^{2}=.07$, adjusted $R^{2}=.05, F(3,139)=3.41, p$ $=.019$, and in this model, the CBAS total $\times$ stress interaction term was significant, $B=$ $0.0003, \beta=.98, t(139)=2.49, p=.014, \mathrm{BCa} 95 \% \mathrm{CI}[0.0001,0.0005]$, indicating that stress moderated the relationship between CBAS total and proportion of specific AMT responses. The third step of the model involving CBAS-B was significant, $R^{2}=.06$, adjusted $R^{2}=.04$, $F(3,139)=2.75, p=.045$, and in this model, the CBAS-B $\times$ stress interaction term was significant, $B=0.0004, \beta=.77, t(139)=2.18, p=.031$, BCa 95\% CI [0.00006, 0.0008], indicating that stress moderated the relationship between CBAS-B and proportion of specific AMT responses. These significant moderations are discussed in their own section below.

When predicting proportion of overgeneral responses, the third steps of the models were not significant, $F_{\mathrm{S}}=0.23$ to $0.65, \mathrm{ps}=.585$ to .879 .

Moderation of the relationship between CBAS and proportion of specific memories. I interpreted only the CBAS total score $\times$ stress interaction, and forwent interpreting the $\mathrm{CBAS}-\mathrm{B} \times$ stress interaction. This was because the CBAS-B is part of the CBAS total score and was likely driving the CBAS total score effect. ModGraph (Jose, 2013) was used to graph the moderation (Figure 6.1). Simple slopes analyses indicated that there was a nonsignificant relationship between CBAS total and proportion of specific responses when stress was high, $t(140)=0.01, p=.991$. There were significant relationships between CBAS total and proportion of specific responses when stress was at a medium, $t(140)=-2.08, p=.039$, or low level, $t(140)=-3.09, p=.002$. This indicated that higher CBAS avoidance was associated with a lower proportion of specific responses, but only when stress was at a low or medium level and not when stress was at a high level. This did not support the prediction that compared to those participants lower on avoidance, those higher on avoidance would exhibit a lower proportion of specific memories and higher proportion of overgeneral memories on the AMT, but only when self-reported levels of stress are high. 


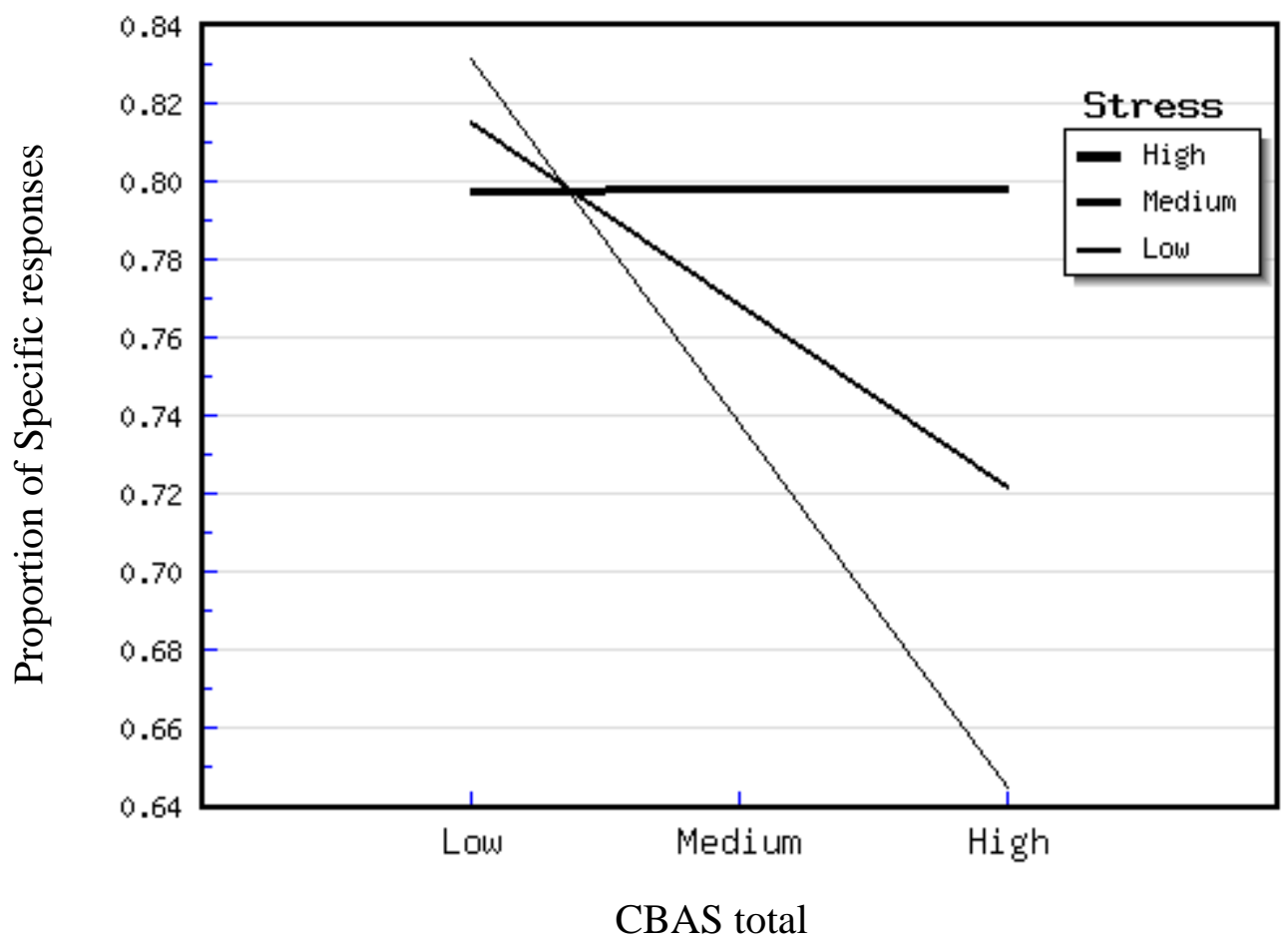

Figure 6.1. Moderation by stress of the relationship between CBAS total score and proportion of specific AMT responses in Study 4.

Cue valence and pleasantness of remembered events. The hypothesis that positive and negative cues would elicit memories for pleasant and unpleasant events, respectively was tested in the PR condition only because in the NPR condition, no pleasantness ratings were made. Pleasantness ratings to positive cues were higher than the pleasantness rating scale's midpoint (3), a statistically significantly mean difference of 1.11 , BCa 95\% CI [1.01, 1.21], $t(72)=21.78, p<.001$. Pleasantness ratings to negative cues were lower than the midpoint, a statistically significant mean difference of -1.26 , BCa 95\% CI $[-1.36,-1.16], t(72)=-25.26, p$ $<.001$.

The mean difference in pleasantness ratings to positive and negative cues was 2.37 BCa 95\% CI [2.21, 2.51]. This difference was significant, $t(72)=30.70, p<.001$. These results supported the hypothesis that positive and negative cues would elicit memories for pleasant and unpleasant events, respectively. Further examination of these pleasantness rating data are presented in Chapter 8.

\section{Discussion}

The aim of Study 4 was to investigate whether the addition of the pleasantness rating scale to the AMT influenced those people higher on avoidance to respond more specifically as compared to those lower on avoidance and as compared to a version of the AMT without a 
pleasantness rating scale. In the pleasantness rating condition, participants were asked to rate the pleasantness of their remembered events on the AMT. In the no pleasantness rating condition, participants did not rate the pleasantness of remembered events. There were no interactions between condition and avoidance measures in predicting proportion of specific or overgeneral AMT responses. In summary, Study 4 showed that rating pleasantness does not interact with avoidance in predicting proportion of specific or overgeneral AMT responses.

In this chapter's introduction, a theoretical rationale was presented for how pleasantness ratings could have influenced avoiders to remember more specifically (as in Studies 1 and 2). I argued that the presence of the pleasantness rating scale on each page may have acted as a compound-cue, influencing the way cues were elaborated. In addition, the instructions may have alerted participants higher on avoidance to the possibility of encountering unpleasant mnemonic material, thus invoking attempts at thought suppression. Because of the potentially higher cognitive load on avoidant participants, an increased likelihood of the occurrence of the to-be-suppressed material was expected. Study 4 findings did not support these ideas.

As with Studies 1, 2, and 3, Study 4 tested hypotheses pertaining to the relationships between stress, avoidance and AMT indices. As with the previous studies, the interaction between each avoidance measure and stress in predicting AMT indices was tested separately. Results indicated that as CBAS avoidance increased, proportion of specific responses decreased, but only when stress was at a low or medium level and not when stress was at a high level. This result is at odds with the general finding in previous research (e.g., Debeer et al., 2012), that as stress increases, more highly avoidant people tend to become less specific in the way they remember. On the contrary, this finding indicated that as stress decreases, more highly avoidant people tend to be less specific in the way they remember. This finding should be interpreted with caution however, as this particular hypothesis was tested many times across the current studies, without significant results.

As with Studies 1, 2, and 3, the basic bivariate relationships between avoidance measures and AMT indices were tested. Effect sizes were small or near-zero, had confidence intervals that always included zero, and that did not reach statistical significance. 


\section{Chapter 7}

\section{Research Synthesis: Meta-Analyses of the Relationships between Avoidance and AMT Indices}

\section{Introduction}

In this chapter I report a quantitative research synthesis to aid in a holistic interpretation of results across Studies that engaged non-clinical samples. The size and direction of the bivariate relationships between avoidance measures and AMT indices differed across these studies to quite an extent. Therefore, to aid in the interpretation of these results, I used meta-analysis for the quantitative synthesis of the correlations between avoidance measures and AMT indices across these studies. The purpose of doing this was to answer a core question of the current thesis: Is overgeneral memory part of a wider repertoire of avoidance strategies?

Meta-analyses generate summary effect sizes for groups of similar studies. Although this approach is widely used to synthesise large numbers of studies (see Lipsey \& Wilson, 2001), it is also recommended for synthesising small numbers of studies (Cumming \& CalinJageman, 2016). The effect-sizes I examined were the correlations between the avoidance measures (AAQ-II, CBAS total, CBAS-B, CBAS-C, and WBSI) and AMT indices (proportion of specific responses, proportion of overgeneral responses), yielding ten metaanalyses. By using meta-analysis to synthesise the results in this way, I provided a more precise estimate of the effect sizes.

Lipsey and Wilson (2001) described the "apples and oranges" (p. 2) problem in metaanalysis; they stated that studies included in a meta-analysis should "be conceptually comparable, that is, deal with the same constructs and relationships" (p. 2). Based on this, I included only Studies 1, 2, and 4 in each meta-analyses. Study 3 was excluded for three reasons. First, whereas Study 1, 2, and 4 were selected from undergraduate research participant pools, Study 3's participants were selected from a counselling service and on the basis of responding affirmatively to the statement "currently experiencing depressed mood". Second, the selection procedure for Study 3 relaxed the constraint used in Studies 1, 2, and 4 that participants should be between 18 and 20 years old; that is, Study 4 participants were not selected on the basis of age. Third, the mechanisms underlying the relationship between avoidance and autobiographical memory specificity may differ in depressed vs. nondepressed populations (Gutenbrunner et al., 2017b; Sumner, 2012). Thus, the meta-analyses 
addressed Research Question 1a outlined in Chapter 1: the investigation of the basic relationship between avoidance and memory specificity in non-clinical groups.

\section{Method}

MetaXL (Version 5.3; EpiGear International Pty Ltd, 2016) was used to run the metaanalyses, and ESCI software (Cumming \& Calin-Jageman, 2016) was used to graph results. A random-effects (RE) model was used for each meta-analysis.

\section{Results}

Summary effect sizes and their 95\% CIs were calculated. Table 7.1 and Table 7.2 display these for proportion of specific and overgeneral AMT responses, respectively, and are depicted in forest plots in Figure 7.1 and Figure 7.2, respectively. In addition, summary effect sizes for all meta-analyses are shown in Figure 7.3, top panel.

Measures of heterogeneity (Cochran's $Q$ and $I^{2}$ ) for each meta-analysis were calculated. $Q$ is a measure of heterogeneity, or variability among the study effect sizes in an individual meta-analysis. $I^{2}$ is also a measure of heterogeneity; it is the percentage of the total variability in effect sizes across studies explained by real differences in the population. In other words, it is a measure of variability not explained by sampling variability. In the following results and interpretation, I discuss heterogeneity with reference to $I^{2} ; Q$ and its associated $p$ values were reported for completeness, but I did not interpret these as they are known to be poor at detecting heterogeneity in meta-analyses (J. P. T. Higgins, Thompson, Deeks, \& Altman, 2003).

Note that in this chapter, there are small discrepancies between the CIs as they are graphed for each Study and the figures reported in previous chapters for those studies. This is because ESCI accepts a sample size and effect size for each study as input and calculates a CI for that study in the graph without bootstrapping. The CIs reported in previous chapters used bootstrapping, however. Summary effect sizes (Pearson's $r$ ) calculated were interpreted according to Lipsey and Wilsons's (2001) guidelines: 0.10 to 0.25 "small", 0.25 to 0.40 "medium", 0.40 and above "large".

\section{Meta-analyses for relationships between avoidance measures and proportion of} specific AMT responses. As can be seen in Table 7.1 and Figure 7.1, effect sizes were all close to zero, and the associated CIs all included zero as a plausible value. Overall, these results indicated that irrespective of the measure of avoidance (AAQ, CBAS total, CBAS-B, CBAS-C, or WBSI), there was no relationship between avoidance and proportion of specific memories. In other words, people high on avoidance did not tend to remember any more or 
less specifically than people low on avoidance.

Table 7.1

Relationships between Proportion of Specific AMT Responses and Avoidance Measures in Meta-Analyses of Studies 1, 2, and 4.

\begin{tabular}{cccc}
\hline & $\begin{array}{c}\text { Summary } \\
\text { Avoidance } \\
\text { effect-size }(r)\end{array}$ & $\begin{array}{c}I^{2} \\
{[95 \% \mathrm{CI}]}\end{array}$ & $\begin{array}{c}\text { Measure } \\
{[95 \% \mathrm{CI}]}\end{array}$ \\
\hline AAQ-II & .04 & $2.11, \mathrm{p}=.348$ & 5 \\
& {$[-.07, .15]$} & & {$[0,90]$} \\
CBAS total & .005 & $6.42, \mathrm{p}=.040$ & 69 \\
& {$[-.18, .20]$} & & {$[0,91]$} \\
CBAS-B & .01 & $4.57, \mathrm{p}=.102$ & 56 \\
& {$[-.15, .17]$} & & {$[0,88]$} \\
CBAS-C & -.002 & $5.79, \mathrm{p}=.055$ & 65 \\
& {$[-.18, .18]$} & & {$[0,90]$} \\
WBSI & .03 & $4.96, \mathrm{p}=.084$ & 60 \\
& {$[-.14, .20]$} & & {$[0,89]$}
\end{tabular}

Note. For all meta-analyses, $d f=2$ and $N=354$. Values for $I^{2}$ are percentages. AAQII $=$ Acceptance and Action Questionnaire II; CBAS = Cognitive Behavioral Avoidance Scale; CBAS-B = CBAS behavioural subscale CBAS-C = CBAS cognitive subscale; WBSI = White Bear Suppression Inventory.

I used guidelines from J. P. T. Higgins, Thompson, Deeks, \& Altman (2003) for interpreting the size of $I^{2}$. With the exception of the AAQ-II meta-analysis, $I^{2}$ was moderate for each meta-analysis. This suggested that chance (sampling variability) could not alone account for differences in effect sizes across studies for CBAS total, CBAS-B, CBAS-C, and WBSI meta-analyses. In contrast, $I^{2}$ for the AAQ-II meta-analysis was low, indicating that total variability across studies was likely due to chance rather than real differences in the population. A cautionary note is needed here. $I^{2}$ can be biased when the number of studies in a meta-analysis is few; in these situations, interpretation should be made with reference to the CIs around $I^{2}$ (von Hippel, 2015). For all meta-analyses reported here, CIs around $I^{2}$ were extremely wide; all lower limits were $0 \%$, and all upper limits were $>87 \%$ (i.e., the plausible 
values for $I^{2}$ were anywhere from low to high for each meta-analysis). This made it difficult to interpret heterogeneity across studies.

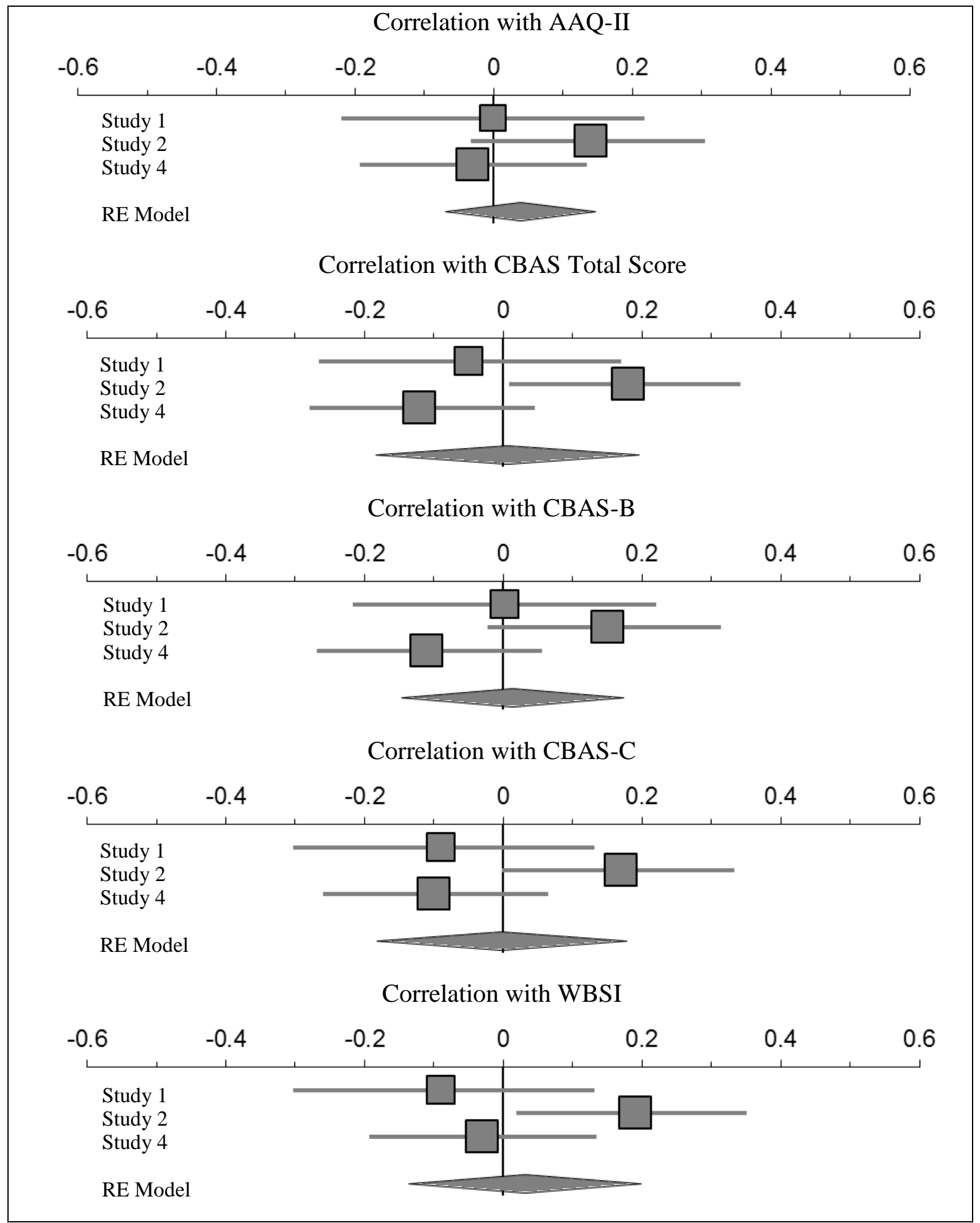

Figure 7.1. Forest Plots of Random-Effects (RE) Meta-Analyses Depicting Correlations (Pearson's $r \mathrm{~s}$ ) between Avoidance Measures and AMT Proportion of Specific Memories. The $\mathrm{x}$-axes are Pearson's $r$. Lines extending either side of a square are the $95 \%$ CI. The size of each square indicates the weighting given to that study in each meta-analysis. The diamonds 
indicate the results of each meta-analysis with the width of the diamond indicating the $95 \%$ CI.

Meta-analyses for relationships between avoidance measures and proportion of general AMT responses. Summary effect sizes and measures of heterogeneity for these meta-analyses are displayed in Table 7.2. Forest plots are displayed in Figure 7.2. A notable observation across meta-analyses is the consistency with which the summary effect sizes are negative, or, as visualised in the forest plots (Figure 7.2), the diamonds are positioned leftward of zero on the x-axes. Overall, the pattern suggests higher self-reported avoidance was associated with a lower proportion of overgeneral AMT responses. A serious challenge to this interpretation, however, is that the CIs around the summary effect sizes either included, or came very close to including, zero. I now discuss the meta-analyses in terms of those with CIs that included zero vs. those that did not.

Summary effect sizes for the AAQ-II, CBAS-B, and WBSI were close to zero or small and their CIs included zero as a plausible value. Thus, levels of avoidance as measured by these (sub)scales were not related to the proportion of overgeneral memories reported on the AMT.

Summary effect sizes for the CBAS total and CBAS-C, however, were small with upper CIs that came very close to including, but did not include, zero. This indicated that people who scored high on these avoidance measures reported a slightly lower proportion of overgeneral AMT responses than people who scored low. This effect was anywhere between small and non-existent, however.

As with the meta-analyses involving proportion of specific responses, total variability across studies not accounted for by sampling variability in these meta-analyses was uncertain: CIs around $I^{2}$ (see Table 6.2) were wide. In other words, it is unclear if it was sampling variability or real differences in the population that accounted for variability between studies in each meta-analysis. 


\section{Table 7.2}

Relationships between Proportion of Overgeneral AMT Responses and Avoidance Measures in Meta-Analyses of Studies 1, 2, and 4.

\begin{tabular}{cccc}
\hline $\begin{array}{c}\text { Avoidance } \\
\text { Measure }\end{array}$ & $\begin{array}{c}\text { Summary } \\
\text { effect-size }(r) \\
{[95 \% \mathrm{CI}]}\end{array}$ & $Q, p$ & $\begin{array}{c}I^{2} \\
{[95 \% \mathrm{CI}]}\end{array}$ \\
\hline AAQ-II & -.08 & $1.32, p=.517$ & 0 \\
& {$[-.18, .02]$} & & {$[0,84]$} \\
CBAS total & -.16 & $3.62, p=.163$ & 45 \\
& {$[-.29,-.01]$} & & {$[0,84]$} \\
CBAS-B & -.11 & $2.26, p=.032$ & 12 \\
& {$[-.22, .004]$} & & {$[0,91]$} \\
CBAS-C & -.17 & $3.89, p=.143$ & 49 \\
& {$[-.31,-.02]$} & & {$[0,85]$} \\
WBSI & -.08 & $9.01, p=.011$ & 78 \\
& {$[-.30, .15]$} & & {$[28,93]$}
\end{tabular}

Note. For all meta-analyses, $d f=2$ and $N=354$. Values for $I^{2}$ are percentages. AAQII $=$ Acceptance and Action Questionnaire II; CBAS = Cognitive Behavioral Avoidance Scale; $\mathrm{CBAS}-\mathrm{B}=\mathrm{CBAS}$ behavioural subscale; $\mathrm{CBAS}-\mathrm{C}=\mathrm{CBAS}$ cognitive subscale; $\mathrm{WBSI}=$ White Bear Suppression Inventory. 


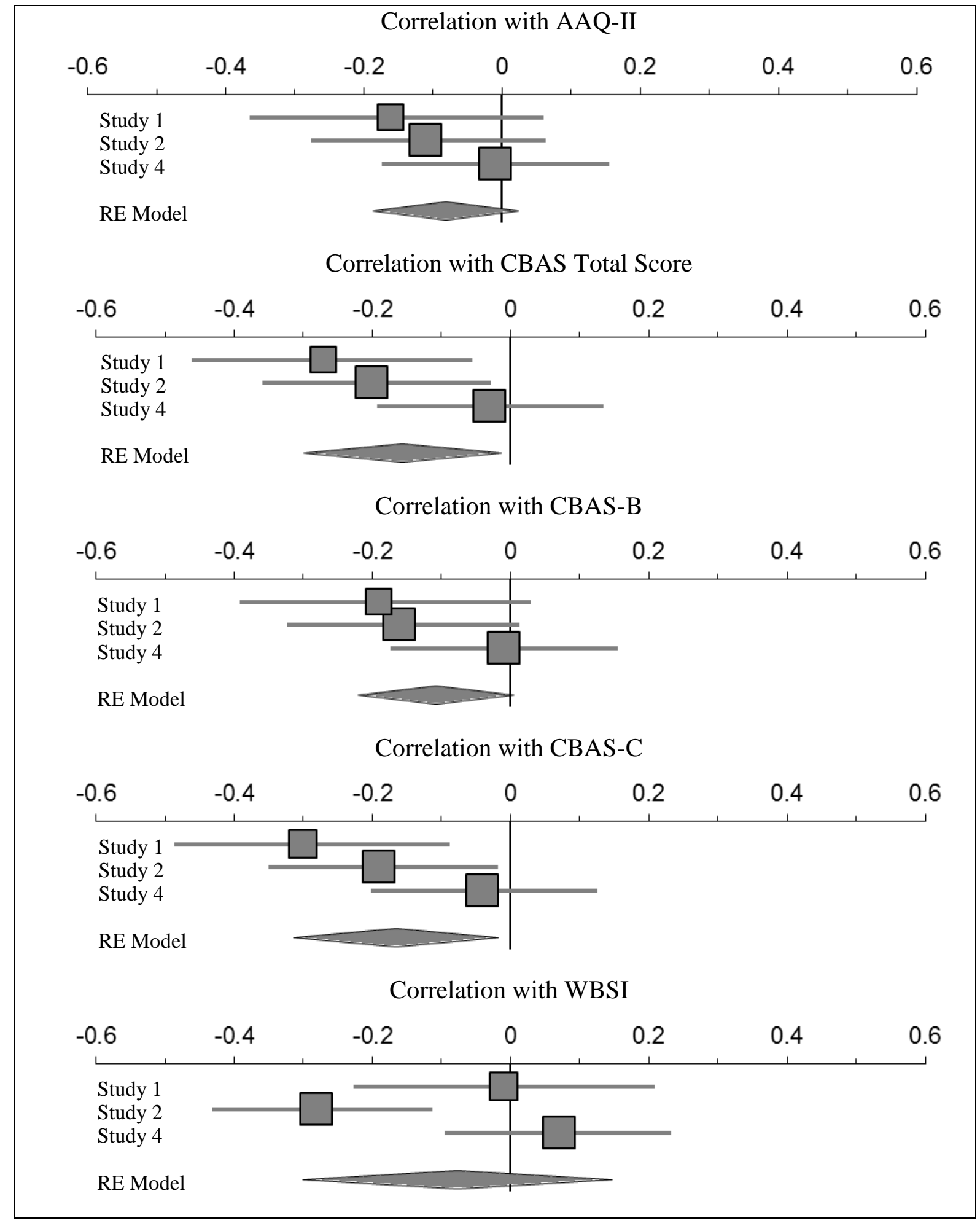

Figure 7.2. Forest Plots of Random-Effects (RE) Meta-Analyses Depicting Correlations (Pearson's $r$ s) between Avoidance Measures and AMT Proportion of Overgeneral Memories. The x-axes are Pearson's $r$. Lines extending either side of a square are the 95\% CI. The size of each square indicates the weighting given to that study in each meta-analysis. The diamonds indicate the results of each meta-analysis with the width of the diamond indicating the $95 \%$ CI. 

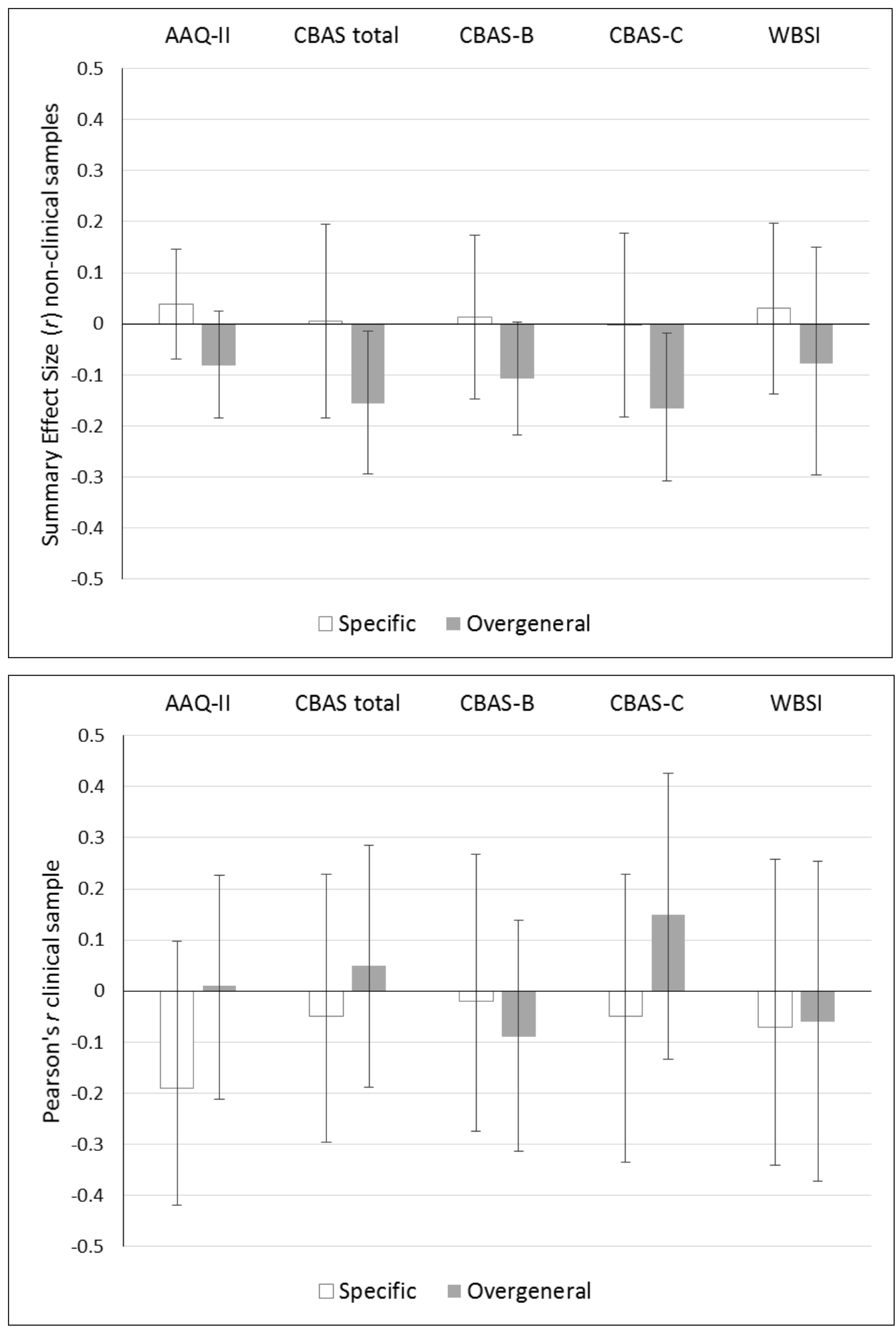

Figure 7.3. Relationships between avoidance measures and proportion of specific and overgeneral memories. Top panel: Meta-analyses of Studies 1, 2, and 4 (non-clinical samples). Bottom panel: Study 3 (clinical sample). Error bars are 95\% CIs. 


\section{Discussion}

In this chapter I described a quantitative research synthesis to aid in a holistic interpretation of results across the non-clinical samples. More specifically, I addressed Research Question 1: the investigation of the relationships between avoidance and memory specificity as it manifests in non-clinical groups. To do this, I calculated a summary effect size for each relationship from Studies 1, 2, and 4 using meta-analyses. Study 3 was excluded from the meta-analysis because it recruited participants from a clinical population.

Based on the results of the meta-analyses (see Figure 7.3 top panel, for a visual summary), I found no support for the idea that remembering a lower proportion of specific memories might be part of a repertoire of avoidance strategies used in non-clinical individuals. On the other hand, I found that avoidance, (specifically, as measured by the CBAS total score and CBAS-C subscale) may be related to remembering a lower proportion of general memories.

Heterogeneity and the possibility of moderators. Overall, the level of heterogeneity in each meta-analysis was uncertain as evidenced by wide CIs around values for $I^{2}$. Put another way, within each meta-analysis, there was uncertainty about the amount of variability across studies that could be accounted for by chance (sampling variability) and that could be accounted for by real differences in the population. The amount of heterogeneity is often uncertain in small meta-analyses (von Hippel, 2015), and this may call into question whether examining heterogeneity is worthwhile when there are few studies, but, as Borenstein et al. (2009) stated, a statistical summary of several studies (i.e., meta-analysis) with high uncertainty should be preferred over one that is ad-hoc (i.e., a qualitative review).

If there was heterogeneity due to sources other than sampling variability, it raises the possibility that there were differences between studies that altered the relationships between variables in non-random ways (i.e., in ways not attributable to sampling variability). In other words, there may be moderator variables that influenced the effect sizes within an individual meta-analysis. Systematic exploration of moderators is advocated in meta-analysis (Card, 2012; Cumming, 2012; Lipsey \& Wilson, 2001). Given the small number of studies in each meta-analysis, however, this was not practical.

Findings in clinical and non-clinical samples. The effect sizes in the non-clinical samples and clinical sample are shown in the top and bottom panels of Figure 7.3, respectively. By juxtaposing visual representations of the relationships in the different sample types like this, I aimed to facilitate interpreting the relationships in the different sample types. 
As can be seen in Figure 7.3, in the non-clinical samples (top panel), the direction of the effect sizes for the relationships between avoidance and proportion of overgeneral memories are consistently negative with most confidence intervals having their upper bounds near zero. On the other hand, in the non-clinical sample, the effect sizes for the relationship between avoidance and proportion of specific memories are weaker and near-zero with confidence intervals that include zero. In the clinical sample (bottom panel), the most salient aspect of the figure is that the confidence intervals always include zero.

I tentatively summarise these findings as follows: In the non-clinical samples, higher avoidance was associated with remembering a lower proportion of specific memories but was not associated with remembering specific memories. In the clinical sample, avoidance and specific and overgeneral remembering was not related. 


\section{Chapter 8}

\section{Pleasantness Ratings of Events Remembered to Positive and Negative Cues}

This chapter is a closer examination of Research Question 3: Do positive and negative cues elicit memories for positive and negative events, respectively? Thus far in this thesis, results indicated the answer to this question is yes; as reported in Ganly et al. (2016) and expanded upon in this thesis, positive and negative cues elicited memories for events rated as pleasant and unpleasant, respectively. These analyses involved examining the mean pleasantness ratings for each participant however, and did not examine the possibility that some pleasantness ratings to individual cues may be incongruent with the cue's valence. In other words, they did not examine if an individual pleasantness rating of an event remembered to a positive cue could be unpleasant, or if an event remembered to a negative cue could be pleasant. To examine this possibility, the percentage of each possible pleasantness rating to positive and negative cues for each study was calculated. For Study 1, percentages were calculated for each Cue-set. These percentages are displayed in Table 8.1 for the AMT and Table 8.2 for the AMT-R.

Table 8.1

Percent of Individual AMT Pleasantness Ratings to Positive and Negative Cues by Study

\begin{tabular}{cccccc}
\hline \multirow{2}{*}{$\begin{array}{c}\text { Pleasantness } \\
\text { rating }\end{array}$} & Cue-set A & Cue-set B & Study 2 & Study 3 & Study 4 \\
\cline { 2 - 5 } & \multicolumn{5}{c}{ Positive cues } \\
1 & 2.4 & 2 & 3.4 & 5.4 & 1.9 \\
2 & 4.9 & 5 & 6.5 & 7.3 & 5.5 \\
3 & 13.7 & 15.5 & 9.8 & 15.4 & 12.6 \\
4 & 34.6 & 30.5 & 26.9 & 30.4 & 34.5 \\
5 & 36.1 & 35.0 & 46.2 & 29.2 & 40.0 \\
Missing & 8.3 & 12.0 & 7.2 & 12.3 & 5.5 \\
\hline & & & Negative cues & & \\
1 & 41.0 & 35.0 & 45.7 & 54.2 & 37.0 \\
2 & 37.6 & 50.0 & 35.7 & 30.0 & 44.9 \\
3 & 7.3 & 3.5 & 7.5 & 4.6 & 8.5 \\
4 & 1.5 & 4.5 & 1.8 & .8 & 1.9 \\
5 & 1.5 & 1.5 & 1.4 & .4 & .5 \\
Missing & 11.2 & 5.5 & 7.8 & 10.0 & 7.1 \\
\hline
\end{tabular}

Note. Imputed values for pleasantness ratings that were non-integers were rounded to the nearest integer. Study 4 data is for the pleasantness rating condition only. 
Table 8.2

Percent of Individual AMT-R Pleasantness Ratings to Positive and Negative Cues in Study 1

\begin{tabular}{ccc}
\hline $\begin{array}{c}\text { Pleasantness } \\
\text { rating }\end{array}$ & Cue-set A & Cue-set B \\
\hline \multicolumn{3}{c}{ Positive cues } \\
1 & 2 & 1.5 \\
2 & 3 & 6.8 \\
3 & 8 & 16.6 \\
4 & 37.5 & 32.7 \\
5 & 40.0 & 33.2 \\
Missing & 9.5 & 9.3 \\
\hline \multicolumn{3}{c}{ Negative cues } \\
1 & 25.5 & 32.2 \\
2 & 50.0 & 42.0 \\
3 & 10.0 & 13.7 \\
4 & 2 & 4.4 \\
5 & 2 & 1.0 \\
Missing & 10.5 & 6.8
\end{tabular}

Note. Imputed values for pleasantness ratings that were non-integers were rounded to the nearest integer.

As can be seen in Tables 8.1 and 8.2, there was a substantial percentage of pleasantness ratings that were rated at the mid-point or incongruent with their cue valence. Although analyses presented in earlier chapters of this thesis confirmed that on average, pleasantness ratings were congruent with their cue valence, the closer examination presented in this chapter indicated that it cannot be assumed that individual positive and negative cues always elicited memories for pleasant and unpleasant events, respectively. 


\section{Chapter 9}

\section{General Discussion}

In this Chapter, I present a summary of findings broken down by research question and a discussion about these findings in the context of previous research. I also discuss limitations, possible future research directions, and clinical implications.

\section{Summary of Findings}

Research Question 1a: What is the bivariate relationship between avoidance and overgeneral memory in non-clinical samples? Previous research (Debeer et al., 2011;

Gibbs \& Rude, 2004) led me to hypothesise that there would be no significant correlation between avoidance and overgeneral memory in non-clinical, undergraduate samples. I found a mixed pattern of findings, however. In the meta-analyses of the undergraduate samples, I found no significant relationship between self-reported avoidance and proportion of specific AMT responses. Experiential avoidance (AAQ-II scores) and thought suppression (WBSI scores) were not significantly correlated with the proportion of general AMT responses. Higher levels of cognitive-behavioural avoidance (CBAS scores) were significantly associated with a lower proportion of general AMT responses. The effect was present for the CBAS total score and its cognitive avoidance subscale (CBAS-C) but not the behavioural avoidance subscale (CBAS-B).

The current studies' findings described above are inconsistent with Hermans et al. (2005) who found that in a group of high-school students, higher scores on the AAQ, WBSI, CBAS total score, and CBAS-B subscales were significantly negatively correlated to the proportion of specific memories on the written AMT. They found no significant relationship with CBAS-C subscales. In addition, they found no significant correlation between any of the avoidance measures and number of categoric or extended responses. Other research (e.g., Gibbs \& Rude, 2004) has found no significant correlations between avoidance and AMT indices. Thus, when considering these studies together, there is a mixed pattern of avoidanceovergenerality findings.

As I put forth in Chapter 4's discussion, one possible way to account for the finding that, at least in the current studies' undergraduate samples, higher avoidance was associated with a lower proportion of general memories on the AMT, is to draw on the ideas in ironic process theory as advanced by Phung and Bryant (2013). They framed functional avoidance as thought suppression and stated that attempts at avoiding specific memories may result in an enhancement effect, an increase in the occurrence of specific memories. Thus, they argued 
the functional avoidance and ironic process theories make opposite predictions about the relationship between avoidance and memory specificity. The current findings partly support the ironic process theory account, and do not support the functional avoidance account. Support for the ironic process theory account is partial because whereas there was a significant relationship between avoidance (as measured by the CBAS total score and CBAS$\mathrm{C}$ subscale) and overgeneral remembering, the relationship with specific remembering was not significant. As pointed out by Debeer et al. (2012), significant findings with only one AMT index, such as in the current studies, are not uncommon in this literature. It is worth noting, however, that even though the thought suppression account may partially explain the current findings, the correlations between AMT indices and the self-report measure of thought suppression (WBSI) did not reach statistical significance. Importantly, some research has shown that actual suppression (as measured in the laboratory) is not predicted by selfreported thought suppression ability or success (Koster, Soetens, Braet, \& De Raedt, 2008). A more appropriate paradigm in future research might therefore involve an objective measure of the number of occurrences of a particular to-be-suppressed thought, and evaluating the degree to which this is related to overgeneral memory.

Several methodological issues are worth exploring with respect to the current findings. First, AMT cues used in the current studies were not the same as those used in the above mentioned studies; there is no one standard set of cue words for the AMT (see Griffith et al., 2012), and individual cue-sets may have idiosyncratic properties (Griffith et al., 2009). It is therefore possible that the emergence of particular relationships between specificity and avoidance may depend to some extent on the AMT cues used. Earlier in this thesis, I described how ironic process theory can be used to explain why avoiding specific memories could make them more likely (Phung \& Bryant, 2013). In short, thought suppression is comprised of the operating and monitoring processes. The operating process searches for material to distract the person from to-be-suppressed thoughts. Simultaneously, the monitoring process inspects consciousness for traces of to-be-suppressed thoughts so that the operating process can be re-engaged if it fails (such as when there are competing cognitive demands). AMT cues used in the current studies such as failure, successful, proud, and regret may have readily cued thoughts of academic achievement and performance experiences (i.e., exam success or failure and the accompanying pride or regret). In other words, for this participant group, these cues may have been highly predicable (Jones, 1998; Williams, Healy, \& Ellis, 1999). Predicability refers to how easily a person can retrieve relevant 
semantic (rather than episodic) information about what the cue refers to. It is possible that these cues, because of their relevance to the ongoing concerns and preoccupations of the university student participants, may have been enough to cause the operating process to fail (i.e., the search for distractors was overwhelmed because of the presence of self-relevant cues). This effect may have influenced participants who tended to engage in avoidance (i.e., thought suppression) to a greater degree than participants who tended not to engage in avoidance.

The second methodological issue that bears on the current findings is that I administered the traditional instructions version of the AMT in which participants are explicitly asked to remember specific memories. Compared to the minimal instructions version (Mi-AMT) which has no explicit instruction to be specific, the traditional instructions version is less sensitive at detecting overgeneral memory in non-clinical samples (Debeer et al., 2009). On the other hand, there is some doubt as to whether the AMT and the Mi-AMT measure the same construct. Because of its specificity instruction, it is reasonable to suggest the traditional instructions version measures the ability to remember specific memories. The Mi-AMT on the other hand, may be a measure of the tendency to do so (Debeer et al.). It is possible that whereas avoidant participants have a tendency to remember fewer specific memories, in the current samples, this tendency was overridden by the specificity instruction. In short, it is possible that although an avoidant person might tend to retrieve fewer specific memories, the traditional-instructions AMT is not an appropriate instrument for measuring it. Future research should investigate what measures are most appropriate for measuring overgeneral memory in both clinical and non-clinical groups.

As highlighted by many commentators (e.g., Cumming, 2012), replication is vital to science. With that in mind, and with respect to the investigation of correlations between avoidance and AMT indices, although not novel, the current examination of this basic relationship constituted important replications of previous research (e.g., Hermans et al., 2005).

\section{Research Question 1b: Does self-reported stress moderate the relationship} between avoidance and overgeneral memory in non-clinical samples? I found very weak evidence of a moderating role of stress in the relationship between overgeneral memory and avoidance in undergraduate or clinical samples; that is, in general, the relationship between avoidance and proportion of specific or general AMT memories did not depend on the level of stress a person reported. In Study 4 there was a significant moderation of the relationship 
between specific memories and CBAS by stress such that higher avoidance was associated with lower specificity but that this only occurred when stress was at a low or medium level, not at a high level. Interpreting this result is difficult given that it was tested many times across the four studies with non-significant results. The overall finding, that there was no moderating role of stress, is in contrast to previous findings that overgeneral memory is a flexible, context-dependent avoidance strategy that is used when an avoidant person is under stress (Debeer et al., 2011, 2012, 2013). What could account for the disparity in findings? Here, I discuss two possibilities.

First, it is possible that the current studies did not capture the type of stress that interacts with avoidance to predict memory specificity. The current studies used the DASS stress scale as a measure of stress. The DASS does not capture a person's momentary emotional state, however; rather, it measures a person's experience of stress over the past week (Lovibond \& Lovibond, 1995). In that way, it differs from stress as it was investigated in previous studies. For example, Debeer et al. (2012) induced stress over an acute time period by having participants carry out a stressful public speaking task, an experience that is perhaps quite different to the type of stress measured by the DASS. It may be that reductions in memory specificity as experienced by avoidant individuals occur only in times of acute stress (i.e., Debeer et al.'s study), rather than in its relatively more chronic form (i.e., the current studies). Future research using a self-report measure more attuned to momentary stress could yield useful results. A further alternative is to assess the occurrence of significant stressful life events directly, perhaps by interviewing participants about events they have encountered recently as well as the their subjective experience of stress associated with those events (e.g., Safford, Alloy, Abramson, \& Crossfield, 2007).

Second, there is a notable point of difference in methodology between the current studies and Debeer et al.'s $(2011,2012,2013)$ work with respect to how overgeneral memory was operationalised. For example, Debeer et al. (2011) measured overgeneral memory at multiple time points and, as well as using scores from individual time points, used change scores in their analyses. The current studies measured overgeneral memory at only one time point, however. Therefore, this thesis is silent on the issue of how an avoidant individual's reduction in memory specificity over time interacts with stress.

If momentary stress is a more appropriate measure of stress, and cross-sectional, as well as change in memory specificity are both important to examine, then a fruitful avenue of future research could be to use an experience-sampling methodology (ESM; 
Csikszentmihalyi \& Larson, 2014). In the ESM paradigm, participants repeatedly record relevant variables over an extended time period as they go about their daily lives. Momentary stress and memory specificity captured in this way could be used to examine patterns of relationships between these variables.

\section{Research question 1c: What are the relationships between avoidance,} overgeneral memory, and stress in a clinical sample? The examination of mechanisms underlying overgeneral memory in different sample types was advocated by Sumner (2012). With this in mind, I examined the relationships between avoidance, overgeneral memory and stress in a sample recruited from a counselling service (clinical sample). There were no significant correlations between avoidance and AMT indices in the clinical sample, and stress did not moderate the relationships between avoidance and AMT indices.

The Study 3 sample was recruited from a cohort of university students who had accessed a university counselling service in the previous year and who also reported currently experiencing depressed mood. As discussed in Chapter 4, it is worth noting that even though the diagnostic-status of these participants was unknown, the mean DASS depression score was in the severe range, a level expected for a diagnosis of depression (Page et al., 2007). Because Study 3 participants had previously sought help (accessed the counselling service), they may have been actively engaging in more adaptive emotion-regulation strategies (i.e., that had been worked on as part of the therapeutic process). It is possible these strategies may have masked or replaced functional avoidance to the extent that the expected pattern of findings did not emerge. In other words, because of the therapeutic process, despite still experiencing low mood, participants in the clinical sample may not have been engaging in functional avoidance.

Overall, these findings addressing research questions $1 \mathrm{a}, 1 \mathrm{~b}$, and 1c suggest that overgeneral memory is not part of a repertoire of avoidance strategies, irrespective of clinical status. These findings also suggest that an avoidance repertoire may include remembering proportionally fewer general memories in non-clinical samples but not in a depressed sample. Stress was not a moderator of the avoidance-overgeneral memory relationship the clinical sample. In the non-clinical samples, there was a suggestion that higher avoidance was associated with lower specificity but that this only occurred when stress was at a medium or low level, and not at a high level. As discussed above, however, this moderation result should be interpreted with caution given the many non-significant tests of this hypothesis. As far as I am aware, this is the only line of research to have examined the relationship between 
avoidance, stress, and AMT performance in non-clinical and clinical samples, and therefore constitutes a novel contribution to knowledge.

\section{Research Question 2: Is remembering a lower proportion of general memories} on the AMT-R associated with higher avoidance? This thesis examined the relationship between avoidance and performance on a reversed version of the AMT (AMT-R) in which participants were asked to remember categoric memories. This was to examine the possibility that there is an overall reduction in autobiographical memory performance (i.e., poorer performance on other tests of memory, not just the AMT) displayed by people higher on avoidance, perhaps because general memories as well as specific memories can be aversive. To test this, in Study 1, in addition to administering the AMT, I administered the AMT-R to all participants. There were no correlations between AMT-R indices and avoidance. In other words, high and low avoiders did not differ in the extent to which they perform on the AMTR. This result is relevant in light of Dritschel, Beltsos, and McClintock's (2014) findings. In a sample of university students, they found rumination was not correlated with the percent of categoric memories retrieved on the AMT-R. Rumination has been conceptualised as a form of avoidance (Nolen-Hoeksema et al., 2008; Watkins, 2016) and in this way, AMT-R results from the current study align with their findings. Taken together, these findings are evidence that the associations between avoidance and overgeneral memory as shown in previous research are not due to a more generalised reduction in autobiographical memory performance.

With respect to AMT-R performance, Dalgleish et al.'s (2007) findings are also relevant. They found that in a sample of adults scoring in the mild to severe range on the BDI, higher depression scores were associated with more specific responses on the AMT-R. In other words, people who were more depressed tended to make more errors on the AMT-R than those who were less depressed. Based on these findings they argued that performance on the AMT-R are dependent to a significant degree on executive control. Future research should examine the possibility that executive control is a mediator between avoidance and AMT-R performance.

A limitation of Study 1's use of the AMT-R, however, is that little is known about what strategies people use when asked to remember categoric memories. Overgeneral memory research has been dominated by the view that on the AMT, emotionally-valenced cue words elicit generative retrieval, the effortful, strategic search of the autobiographical memory knowledge base that proceeds from abstract, general representations of events to 
more event-specific, experience-near representations (Conway \& Pleydell-Pearce, 2000; Williams et al., 2007). Recent research (e.g., Uzer, 2016) has challenged this view, however, indicating that emotional cue words often elicit direct retrieval of specific memories, a relatively less effortful, spontaneous process. This raises the possibility that in Study 1, AMT-R cues may have directly activated specific memories, at odds with the instruction to remember categoric memories. It is therefore interesting to consider what a participant would have done if an AMT-R cue elicited a specific memory. They may, based on the task instructions to retrieve a categoric memory rather than the specific memory they currently have in mind, ask themselves: "Has this specific event that I have in mind happened on more than one occasion?" If they can answer yes, then they can report their memory in such a way as to reflect a categoric representation, belying the fact that they in fact experienced retrieval of a specific memory. For example, a participant may have remembered: "Two weeks ago on the beach with my friend" which would be coded as a specific memory. Aware of the AMT$\mathrm{R}$ task instructions, however, they may have responded, "The times I've spent on the beach with my friend" which would be coded as a categoric memory. In the hypothetical case just described, retrieval search has proceeded from specific to general rather than general to specific. Moving from a specific to general representation in this way would be opposite to what is predicted by the Self-Memory System (Conway \& Pleydell-Pearce, 2000). If it is the case that the AMT and AMT-R can elicit a similar retrieval strategy (i.e., involving direct retrieval), it may be that the AMT-R is too similar to the AMT to be a useful test of a generalised reduction in memory performance. Future research should therefore investigate what retrieval strategies people use when asked to remember general memories and whether the strategies are distinct from strategies for retrieving specific memories.

It is important to note that the AMT-R was administered only in Study 1 of the present thesis, and these findings should therefore be considered preliminary. Further research involving the relationship between remembering general memories and avoidance should be undertaken. As far as I am aware, this thesis is the first examination of the relationship between avoidance and AMT-R performance and thus constitutes a novel contribution to knowledge.

\section{Research Question 3: Do positive and negative cues elicit memories for positive} and negative events, respectively? The third aim of this thesis was to carry out a methodological check pertaining to AMT cues. Specifically, I examined whether positive and negative cues elicited memories of corresponding valence, that is, memories for positive and 
negative events, respectively. In all four studies, I asked participants to rate the pleasantness of each remembered event on a scale of 1 (extremely unpleasant) to 5 (extremely pleasant). Across all four studies, mean pleasantness ratings of events remembered to positive AMT cues were significantly higher than to negative AMT cues. In addition, mean pleasantness ratings for events remembered to positive and negative cues were in the upper and lower halves of the scale, respectively, and were significantly different from the midpoint of the scale (3). The same pattern emerged for the AMT-R in Study 1. These results indicated that in general, positive and negative cues elicited memories for positive and negative events, respectively. However, additional analyses presented in Chapter 8 indicated that there were numerous AMT and AMT-R responses with pleasantness ratings incongruent with their cuevalence.

These findings are interesting to consider with respect to previous research indicating that the AMT has a one-factor structure (Griffith et al., 2009; see also Heron et al., 2012). In psychometric analyses of written and verbal versions of the AMT in one high school sample (mean age 17.1 years old) and two undergraduate samples (mean ages 18.4 years old and 18.1 years old), Griffith et al. showed that memory specificity is best measured when information from positive and negative cues are combined. Based on their findings, Griffith et al. cautioned against interpreting valence effects in which overgeneral memory to positive and negative cues are examined separately. The current studies are further reason to heed this caution; they suggest that positive and negative cues do not reliably elicit memories for pleasant and unpleasant events.

These findings are also interesting in light of Williams et al.'s (2007) point that for functional avoidance to be effective, it would need to be generalised to the whole autobiographical memory knowledge base. Williams et al. stated:

If a person does not want to remember the assault that he or she experienced when visiting an uncle in the summer of 1969, then it does not help to be overgeneral only for cues like trauma or rape. Even positive or neutral words, like holiday or journey, might lead to these specific painful memories. (p. 135)

The current findings show that on the AMT (and AMT-R), positively-valenced cues may elicit memories for unpleasant events (and vice versa), providing empirical support for Williams et al.'s assertion. 
An important limitation with respect to interpreting these pleasantness rating results is that the instructions for the pleasantness rating scale did not include any information about what 2,3 , or 4 on the pleasantness rating scale meant; following Geraerts et al. (2012), only 1 and 5 were given labels: "Extremely unpleasant" and "Extremely pleasant", respectively. Thus, the meaning of, for example, the midpoint on the scale (3) was ambiguous. Its meaning could have been interpreted differently by different participants - it could have meant, for example, "the event was neither pleasant nor unpleasant" or "the event had both pleasant and unpleasant aspects". Future research could address this by allowing for pleasantness and unpleasantness to be rated on separate scales.

As far as a I am aware, this is the first study to examine the pleasantness of remembered events in this way and thus constitutes a unique contribution to knowledge.

\section{Research Question 4: Does rating the pleasantness of events influence memory} specificity on the AMT? In Study 4, I investigated whether rating (vs. not rating) the pleasantness of remembered events influenced the temporal specificity of AMT responses for high and low avoiders in an undergraduate, non-clinical sample. The rationale for this was to try to explain why I found that high avoiders (as measured on the CBAS total score and CBAS-C subscale in the undergraduate samples) gave a lower proportion of general AMT responses. A notable departure from most other research using the AMT was that I had included a pleasantness rating scale on each page of the response booklet. This raised the possibility that it was rating pleasantness that led to the unexpected finding. Based on ideas put forth by Phung and Bryant (2013), I argued that the AMT's instruction to rate the (un)pleasantness of each remembered event may have increased the cognitive load on avoidant participants leading to an enhancement effect (Wenzlaff \& Wegner, 2000) such that their overgenerality decreased. The results do not support this theoretical justification, however.

Study 4's findings should be considered preliminary, however, and replication is needed. In addition, although I found no effect of pleasantness rating using this paradigm, the theoretical justification I made warrants further attention. More specifically, because my theoretical justification posited that increased cognitive load may play a role in any potential enhancement effect, future research could attempt to manipulate cognitive load during completion of the AMT to see whether it differentially effects high vs. low avoiders in their tendency to remember overgeneral memories. 
These findings constitute a novel contribution to knowledge; this was the first study to examine the effect of adding a pleasantness rating scale to the AMT.

\section{Clinical Implications}

The current studies' findings that avoidance and overgeneral memory were unrelated in the clinical sample are interesting to consider in light of recent research suggesting that targeting overgeneral memory could be useful in the treatment of depression (Hitchcock et al., 2016; Neshat-Doost et al., 2013; Raes, Williams, \& Hermans, 2009; Watkins et al., 2009; 2012). Two studies have examined the effect of these interventions on avoidance. Findings from Hitchcock et al. (2016) and Raes et al. (2009) found that helping people who are depressed or remitted from depression to reduce their overgeneral memory reduced their avoidance. Because their studies did not use control groups, making causal inferences is difficult. Nevertheless, their findings suggest that increasing the flexibility of a person' $\mathrm{s}$ autobiographical memory can lead to a reduction in avoidance. Given Study 3' s nonsignificant relationships between avoidance measures and overgeneral memory, however, the relationships between avoidance and the temporal specificity of autobiographical memory warrants more attention in future clinical research.

\section{Conclusions}

In this chapter, I summarised findings from the current studies, limitations to the methodologies used, and made suggestions for future research. Based on these, in this section, I make several broad recommendations for the field of overgeneral memory research going forward.

A main aim of this thesis was to examine the idea that overgeneral memory may be part of a repertoire of avoidance strategies, and to examine this in clinical and non-clinical groups. Bearing on this, I discussed theories of overgeneral memory that predict higher avoidance should be associated with greater overgeneral memory (Williams, 1996; Hermans et al., 2005; Williams et al., 2007), and that this effect will perhaps only be present when levels of stress are high (Debeer et al., 2011). Another theory predicts that avoidance (in particular, thought suppression) should be associated with less overgeneral memory (Phung \& Bryant, 2013). I also presented a justification for why rating the pleasantness of events might influence some participants to remember in less overgeneral ways. Future research should attempt to reconcile these theories, investigating under what circumstances a person's tendency to avoid might result in remembering more or less specifically (i.e., if under stress, if asked to rate pleasantness of memories, if actively suppressing unpleasant memories). 
Methodological issues pertaining to how autobiographical memories can be best elicited from participants crosscut the current research. In particular, there is a need to investigate what tests can effectively measure overgeneral memory's association with other variables (e.g., avoidance) across clinical and non-clinical groups. In addition, an unanswered question is what strategies people use when they are asked to retrieve categoric memories (i.e., on the AMT-R). Future research should compare the strategies people use to recall memories under different instructions (i.e., specificity instruction on the AMT; categoric instruction on the AMT-R; no specificity instruction on the Mi-AMT) and investigate to what extent these strategies are influenced by avoidance. 


\section{References}

Beck, A. T., Steer, R. A,, \& Brown, G. K. (1996). Manual for the Beck Depression InventoryII. San Antonio, TX: Psychological Corporation.

Bernsten, D., \& Rubin, D. C. (2012). Understanding autobiographical memory: An ecological theory. In D. Bernsten \& D. C. Rubin (Eds.), Understanding autobiographical Memory: theories and approaches (pp. 333-355). Cambridge, England: Cambridge University Press.

Bluck, S. (2003). Autobiographical memory: Exploring its functions in everyday life. Memory, 11(2), 113-123. doi:10.1080/741938206

Bluck, S., Alea, N., Habermas, T., \& Rubin, D. C. (2005). A tale of three functions: The self reported uses of autobiographical memory. Social Cognition, 23(1), 91-117. doi:10.1521/soco.23.1.91.59198

Bond, F. W., Hayes, S. C., Baer, R. a., Carpenter, K. M., Guenole, N., Orcutt, H. K., ... Zettle, R. D. (2011). Preliminary psychometric properties of the Acceptance and Action Questionnaire-II: A revised measure of psychological inflexibility and experiential avoidance. Behavior Therapy, 42(4), 676-688. doi:10.1016/j.beth.2011.03.007

Borenstein, M., Hedges, L. V, Higgins, J. P. T., \& Rothstein, H. R. (2009). Introduction to Meta-Analysis. West Sussex, England: Wiley.

Borkovec, T. D., \& Roemer, L. (1995). Perceived functions of worry among generalized anxiety disorder subjects: Distraction from more emotionally distressing topics? Journal of Behavior Therapy and Experimental Psychiatry, 26(1), 25-30. doi:10.1016/00057916(94)00064-S

Bunnell, S. L., \& Greenhoot, A. F. (2012). When and why does abuse predict reduced autobiographical memory specificity? Memory, 20(2), 121-37. doi:10.1080/09658211.2011.648197

Bunnell, S. L., \& Greenhoot, A. F. (2017). Do overgeneral memories make us feel better? An experimental examination. Memory 26(1), 74-88. doi:10.1080/09658211.2017.1323105

Card, N. A. (2012). Applied meta-analysis for social science research. New York, NY: Guilford Press.

Carver, C. S. (2006). Approach, avoidance, and the self-regulation of affect and action. Motivation and Emotion, 30(2), 105-110. doi:10.1007/s11031-006-9044-7

Carver, C. S., \& Scheier, M. F. (1990). Origins and functions of positive and negative affect: A control-process view. Psychological Review, 97(1), 19-35. doi:10.1037/0033- 
295X.97.1.19

Cohen, J. (1992). A power primer. Psychological bulletin, 112(1), 155. doi: 10.1037/00332909.112.1.155

Conway, M. A. (2005). Memory and the self. Journal of Memory and Language, 53(4), 594 628. doi:10.1016/j.jml.2005.08.005

Conway, M. A., \& Jobson, L. (2012). On the nature of autobiographical memory. In D.

Bernsten \& D. C. Rubin (Eds.), Understanding autobiographical memory: Theories and approaches (pp. 54-69). Cambridge, England: Cambridge University Press.

Conway, M. A., \& Loveday, C. (2010). Accessing autobiographical memories. In Mace, J. H. (Ed.) The act of remembering (pp. 56-70). West Sussex, England: Wiley-Blackwell.

Conway, M. A., \& Pleydell-Pearce, C. W. (2000). The construction of autobiographical memories in the self-memory system. Psychological Review, 107(2), 261-288. doi:10.1037/0033-295X.107.2.261

Conway, M. A., Singer, J. A., \& Tagini, A. (2004). The self and autobiographical memory: Correspondence and coherence. Social Cognition, 22(5), 491-529. doi:10.1521/soco.22.5.491.50768

Crane, C., Barnhofer, T., \& Williams, J. M. G. (2007). Cue self-relevance affects autobiographical memory specificity in individuals with a history of major depression. Memory, 15(3), 312-323. doi:10.1080/09658210701256530

Crowne, D., \& Marlowe, D. (1960). A new scale of social desirability independent of psychopathology. Journal Of Consulting Psychology, 24(4), 349-354. doi: $10.1037 / \mathrm{h} 0047358$

Csikszentmihalyi, M., \& Larson, R. (2014). Validity and reliability of the experiencesampling method. In M. Csikszentmihalyi (Ed.), Flow and the foundations of positive psychology: The collected works of Mihaly Csikszentmihalyi (pp. 35-54). Dordrecht, Netherlands: Springer.

Cumming, G. (2012). Understanding the new statistics: Effect sizes, confidence intervals, and meta-analysis. New York, NY: Routledge.

Cumming, G., \& Calin-Jageman, R. (2016). Introduction to the new statistics: Estimation, open science, and beyond. New York, NY: Routledge.

D'Argembeau, A. (2012). Autobiographical memory and future thinking. In D. Bernsten \& D. C. Rubin (Eds.), Understanding autobiographical memory: Theories and approaches (pp. 311-330). Cambridge, England: Cambridge University Press. 
D’Zurilla, T. J., \& Nezu, A. M. (1999). Problem-solving therapy: A social competence approach to clinical intervention. (2nd ed.). New York, NY: Springer Publishing Co.

Dalgleish, T., Williams, J. M. G., Golden, A.-M. J., Perkins, N., Barrett, L. F., Barnard, P. J., ... Watkins, E. (2007). Reduced specificity of autobiographical memory and depression: The role of executive control. Journal of Experimental Psychology: General, 136(1), 23-42. doi:10.1037/0096-3445.136.1.23

Dalgleish, T., \& Yiend, J. (2006). The effects of suppressing a negative autobiographical memory on concurrent intrusions and subsequent autobiographical recall in dysphoria. Journal of Abnormal Psychology, 115(3), 467-473. doi:10.1037/0021-843X.115.3.467

Debeer, E., Hermans, D., \& Raes, F. (2009). Associations between components of rumination and autobiographical memory specificity as measured by a Minimal Instructions Autobiographical Memory Test. Memory, 17(8), 892-903. doi:10.1080/09658210903376243

Debeer, E., Raes, F., Claes, S., Vrieze, E., Williams, J. M. G., \& Hermans, D. (2012). Relationship between cognitive avoidant coping and changes in overgeneral autobiographical memory retrieval following an acute stressor. Journal of Behavior Therapy and Experimental Psychiatry, 43(SUPPL. 1), S37-S42. doi:10.1016/j.jbtep.2011.04.002

Debeer, E., Raes, F., Williams, J. M. G., \& Hermans, D. (2011). Context-dependent activation of reduced autobiographical memory specificity as an avoidant coping style. Emotion, 11(6), 1500-1506. doi:http://dx.doi.org/10.1037/a0024535

Debeer, E., Raes, F., Williams, J. M. G., \& Hermans, D. (2013). Reduction in memory specificity following an approach/avoidance scrambled sentences task relates to cognitive avoidant coping. The Psychological Record, 63(1), 73-84. doi:10.11133/j.tpr.2013.63.1.006

Dritschel, B., Beltsos, S., \& McClintock, S. M. (2014). An “alternating instructions” version of the Autobiographical Memory Test for assessing autobiographical memory specificity in non-clinical populations. Memory 22(8), 37-41. doi:10.1080/09658211.2013.839710

Ferster, C. B. (1973). A functional analysis of depression. American Psychologist, 28(10), 857-870. doi:10.1037/h0035605

Field, A. P. (2013). Discovering statistics using IBM SPSS Statistics. London: Sage.

Field, A. P., \& Wilcox, R. R. (2017). Robust statistical methods: A primer for clinical psychology and experimental psychopathology researchers. Behaviour Research and 
Therapy, 98, 19-38. doi:10.1016/j.brat.2017.05.013

Ganly, T. J., Salmon, K., \& McDowall, J. (2016). Is remembering less specifically part of an avoidant coping style? Associations between memory specificity, avoidant coping, and stress. Cognition and Emotion, 31(7), 1419-1430. doi:10.1080/02699931.2016.1227304

Geraerts, E., Dritschel, B., Kreplin, U., Miyagawa, L., \& Waddington, J. (2012). Reduced specificity of negative autobiographical memories in repressive coping. Journal of Behavior Therapy and Experimental Psychiatry, 43(SUPPL. 1), S32-S36. doi:10.1016/j.jbtep.2011.05.007

Gibbs, B. R., \& Rude, S. S. (2004). Overgeneral autobiographical memory as depression vulnerability. Cognitive Therapy and Research, 28(4), 511-526. doi:10.1023/B:COTR.0000045561.72997.7c

Goddard, L., Dritschel, B., \& Burton, a. (1996). Role of autobiographical memory in social problem solving and depression. Journal of Abnormal Psychology, 105(4), 609-616. doi:10.1037/0021-843X.105.4.609

Gray, J. A., \& McNaughton, N. (2000). The neuropsychology of anxiety: An enquiry into the functions of the septo-hippocampal system (2nd ed.). New York, NY: Oxford University Press.

Griffith, J. W., Sumner, J. A., Debeer, E., Raes, F., Hermans, D., Mineka, S., .. Craske, M. G. (2009). An item response theory/confirmatory factor analysis of the Autobiographical Memory Test. Memory, 17(6), 609-623. doi:10.1080/09658210902939348

Griffith, J. W., Sumner, J. A., Raes, F., Barnhofer, T., Debeer, E., \& Hermans, D. (2012). Current psychometric and methodological issues in the measurement of overgeneral autobiographical memory. Journal of Behavior Therapy and Experimental Psychiatry, 43(SUPPL. 1), 21-31. doi:10.1016/j.jbtep.2011.05.008

Gutenbrunner, C., Salmon, K., \& Jose, P. E. (2017a). Do overgeneral autobiographical memories predict increased psychopathological symptoms in community youth? A 3year longitudinal investigation. Advance Online Publication. doi:10.1007/s10802-0170278-5

Gutenbrunner, C., Salmon, K., \& Jose, P. E. (2017b). What predicts overgeneral memory in youth? Testing the CaR-FA-X model longitudinally in community adolescents. Manuscript submitted for publication.

Hayes, S. C., Barnes-Holmes, D., \& Roche, B. (Eds.). (2001). Relational frame theory: A post-Skinnerian account of human language and cognition. New York, NY: Kluwer 
Academic/Plenum Publishers.

Hayes, S. C., Strosahl, K. D., \& Wilson, K. G. (2012). Acceptance and commitment therapy: The process and practice of mindful change (2nd ed.). New York, NY: Guilford Press. Hayes, S. C., Strosahl, K., Wilson, K. G., Bissett, R. T., Pistorello, J., Toarmino, D., ... McCurry, S. M. (2004). Measuring experiential avoidance: A preliminary test of a working model. The Psychological Record, 54(4), 553-578. doi: 10.1007/BF03395492 Hayes, S. C., Wilson, K. G., Gifford, E. V, Follette, V. M., \& Strosahl, K. (1996).

Experiential avoidance and behavioral disorders: A functional dimensional approach to diagnosis and treatment. Journal of Consulting and Clinical Psychology, 64(6), 11521168. doi:10.1037/0022-006X.64.6.1152

Hermans, D., De Decker, A., De Peuter, S., Raes, F., Eelen, P., \& Williams, J. M. G. (2008). Autobiographical memory specificity and affect regulation: Coping with a negative life event. Depression and Anxiety, 25(9), 787-792. doi:10.1002/da.20326

Hermans, D., Defranc, A., Raes, F., Williams, J. M. G., \& Eelen, P. (2005). Reduced autobiographical memory specificity as an avoidant coping style. The British Journal of Clinical Psychology, 44, 583-589. doi:10.1037/a0024535

Heron, J., Crane, C., Gunnell, D., Lewis, G., Evans, J., \& Williams, J. M. G. (2012). 40,000 memories in young teenagers: Psychometric properties of the Autobiographical Memory Test in a UK cohort study. Memory, 20(3), 300-320. doi:10.1080/09658211.2012.656846

Higgins, E. T. (1987). Self-discrepancy: A theory relating self and affect. Psychological Review, 94(3), 319-340. doi:10.1037/0033-295X.94.3.319

Higgins, E. T. (1997). Beyond pleasure and pain. American Psychologist, 52(12), 1280-300. doi:10.1037/0003-066X.52.12.1280

Higgins, J. P. T., Thompson, S. G., Deeks, J. J., \& Altman, D. G. (2003). Measuring inconsistency in meta-analyses. British Medical Journal, 327(7414), 557-560. doi:10.1136/bmj.327.7414.557

Hitchcock, C., Mueller, V., Hammond, E., Rees, C., Werner-Seidler, A., \& Dalgleish, T. (2016). The effects of autobiographical memory flexibility (MemFlex) training: An uncontrolled trial in individuals in remission from depression. Journal of Behavior Therapy and Experimental Psychiatry, 52, 92-98. doi:10.1016/j.jbtep.2016.03.012 Horowitz, M., Wilner, N., \& Alvarez, W. (1979). Impact of Event Scale: A measure of subjective stress. Psychosomatic Medicine, 41(3), 209-218. doi:10.1097/00006842- 
197905000-00004

Howell, D. C. (2013). Statistical Mmethods for psychology (8th ed.). Belmont, CA:

Wadsworth, Cengage Learning.

Jones, G. V. (1988). Images, predicates, and retrieval cues. In M. Denis, J. Engelkamp, \& J. T. E. Richardson (Eds.), Cognitive and neuropsychological approaches to mental imagery (pp. 89-98). Dordrecht, Netherlands: Springer Netherlands. doi:10.1007/97894-009-1391-2_8

Jose, P.E. (2013). ModGraph-I: A programme to compute cell means for the graphical display of moderational analyses: The internet version, Version 3.0. Victoria University of Wellington, Wellington, New Zealand. Retrieved from https://psychology.victoria.ac.nz/modgraph/

Klein, S. B. (2013). The temporal orientation of memory: It's time for a change of direction. Journal of Applied Research in Memory and Cognition, 2(4), 222-234. doi:10.1016/j.jarmac.2013.08.001

Koster, E. H. W., Soetens, B., Braet, C., \& De Raedt, R. (2008). How to control a white bear? Individual differences involved in self-perceived and actual thought-suppression ability. Cognition and Emotion, 22(6), 1068-1080. doi:10.1080/02699930701616591

Krohne, H. W., Egloff, B., Varner, L. J., Burns, L. R., Weidner, G., \& Ellis, H. C. (2000). The assessment of dispositional vigilance and cognitive avoidance: Factorial structure, psychometric properties, and validity of the Mainz Coping Inventory. Cognitive Therapy and Research, 24(3), 297-311. doi:10.1023/A:1005511320194

Kuyken, W., \& Dalgleish, T. (1995). Autobiographical memory and depression. British Journal of Clinical Psychology, 34(1), 89-92. doi:10.1111/j.2044-8260.1995.tb01441.x

Kuyken, W., Howell, R., \& Dalgleish, T. (2006). Overgeneral autobiographical memory in depressed adolescents with, versus without, a reported history of trauma. Journal of Abnormal Psychology, 115(3), 387-396. doi:10.1037/0021-843X.115.3.387

Lazarus, R. S., Delongis, A., Folkman, S., \& Gruen, R. (1985). Stress and adaptional outcomes: The problem of confounded measures. American Psychologist, 40(7), 770779. doi:10.1037/0003-066X.40.7.770

Lipsey, M. W., \& Wilson, D. B. (2001). Practical meta analysis. Thousand Oaks, CA: Sage Publications.

Lovibond, S. H., \& Lovibond, P. F. (1995). Manual for the Depression Anxiety Stress Scales (2nd Ed.). Sydney, Australia: Psychology Foundation. 
Magee, J. C., Harden, K. P., \& Teachman, B. A. (2012). Psychopathology and thought suppression: A quantitative review. Clinical Psychology Review, 32(3), 189-201. doi:10.1016/j.cpr.2012.01.001

Mansell, W., Harvey, A., Watkins, E., \& Shafran, R. (2009). Conceptual foundations of the transdiagnostic approach to CBT. Journal of Cognitive Psychotherapy, 23(1), 6-19. doi:10.1891/0889-8391.23.1.6

McEvoy, P. M., Thibodeau, M. A., \& Asmundson, G. J. G. (2014). Trait repetitive negative thinking: A brief transdiagnostic assessment. Journal of Experimental Psychopathology, 5(3), 382-398. doi:10.5127/jep.037813

McEwen, B. S. (2007). Physiology and neurobiology of stress and adaptation: Central role of the brain. Physiological Reviews, 87(3), 873-904. doi:10.1152/physrev.00041.2006

MetaXL (Version 5.3) [Computer software]. (n.d.). Noosa, Australia: EpiGear International Pty Ltd.

Moore, S. A, \& Zoellner, L. A. (2007). Overgeneral autobiographical memory and traumatic events: An evaluative review. Psychological Bulletin, 133(3), 419-437. doi:10.1037/0033-2909.133.3.419

Moos, R. H., \& Schaefer, J. A. (1993). Coping resources and processes: Current concepts and measures. In L. Goldberger \& S. Breznitz (Eds.), Handbook of stress: Theoretical and clinical aspects (pp. 234-257). New York, NY: Free Press.

Moulds, M. L., \& Kandris, E. (2007). The nature and role of avoidance in depression. In D. A. Einstein (Ed.), Innovations and advances in cognitive behaviour therapy (pp. 215223). Bowen Hills, Australia: Australian Academic Press.

Moulds, M. L., Kandris, E., Starr, S., \& Wong, A. C. M. (2007). The relationship between rumination, avoidance and depression in a non-clinical sample. Behaviour Research and Therapy, 45(2), 251-261. doi:10.1016/j.brat.2006.03.003

Neshat-Doost, H. T., Dalgleish, T., Yule, W., Kalantari, M., Ahmadi, S. J., Dyregrov, A., \& Jobson, L. (2013). Enhancing autobiographical memory Specificity through cognitive training: An intervention for depression translated from basic science. Clinical Psychological Science, 1(1), 84-92. doi:10.1177/2167702612454613

Neufeind, J., Dritschel, B., Astell, A. J., \& MacLeod, M. D. (2009). The effects of thought suppression on autobiographical memory recall. Behaviour Research and Therapy, 47(4), 275-284. doi:10.1016/j.brat.2008.12.010

Nolen-Hoeksema, S., Wisco, B. E., \& Lyubomirsky, S. (2008). Rethinking rumination. 
Perspectives on Psychological Science, 3(5), 400-424. doi:10.1111/j.17456924.2008.00088.x

Ottenbreit, N. D., \& Dobson, K. S. (2004). Avoidance and depression: The construction of the Cognitive-Behavioral Avoidance Scale. Behaviour Research and Therapy, 42(3), 293-313. doi:10.1016/S0005-7967(03)00140-2

Page, A. C., Hooke, G. R., \& Morrison, D. L. (2007). Psychometric properties of the Depression Anxiety Stress Scales (DASS) in depressed clinical samples. British Journal of Clinical Psychology, 46(3), 283-297. doi:10.1348/014466506X158996

Phung, S. Q., \& Bryant, R. a. (2013). The influence of cognitive and emotional suppression on overgeneral autobiographical memory retrieval. Consciousness and Cognition, 22(3), 965-974. doi:10.1016/j.concog.2013.06.008

Quigley, L., Wen, A., \& Dobson, K. S. (2017). Avoidance and depression vulnerability: An examination of avoidance in remitted and currently depressed individuals. Behaviour Research and Therapy, 97, 183-188. doi:http://dx.doi.org/10.1016/j.brat.2017.07.015

Raes, F., Hermans, D., de Decker, A., Eelen, P., \& Williams, J. M. G. (2003). Autobiographical memory specificity and affect regulation: an experimental approach. Emotion, 3(2), 201-206. doi:10.1037/1528-3542.3.2.201

Raes, F., Hermans, D., Williams, J. M. G., \& Eelen, P. (2006). Reduced autobiographical memory specificity and affect regulation. Cognition \& Emotion, 20(3-4), 402-429. doi:10.1080/02699930500341003

Raes, F., Hermans, D., Williams, J. M. G., \& Eelen, P. (2007). A sentence completion procedure as an alternative to the Autobiographical Memory Test for assessing overgeneral memory in non-clinical populations. Memory, 15(5), 495-507. doi:10.1080/09658210701390982

Raes, F., Watkins, E. R., Williams, J. M. G., \& Hermans, D. (2008). Non-ruminative processing reduces overgeneral autobiographical memory retrieval in students. Behaviour Research and Therapy, 46(6), 748-756. doi:10.1016/j.brat.2008.03.003

Raes, F., Williams, J. M. G., \& Hermans, D. (2009). Reducing cognitive vulnerability to depression: A preliminary investigation of MEmory Specificity Training (MEST) in inpatients with depressive symptomatology. Journal of Behavior Therapy and Experimental Psychiatry, 40(1), 24-38. doi:10.1016/j.jbtep.2008.03.001

Reynolds, M., \& Brewin, C. R. (1998). Intrusive cognitions, coping strategies and emotional responses in depression, post-traumatic stress disorder and a non-clinical population. 
Behaviour Research and Therapy, 36(2), 135-147. doi:10.1016/S0005-7967(98)00013-8

Rothman, K. J. (1990). No adjustments are needed for multiple comparisons. Epidemiology, l(1), 43-46. doi:10.1097/00001648-199001000-00010

Safford, S. M., Alloy, L. B., Abramson, L. Y., \& Crossfield, A. G. (2007). Negative cognitive style as a predictor of negative life events in depression-prone individuals: A test of the stress generation hypothesis, 99(1-3), 147-154. doi:10.1016/j.jad.2006.09.003

Smets, J., Griffith, J. W., Wessel, I., Walschaerts, D., \& Raes, F. (2013). Depressive symptoms moderate the effects of a self-discrepancy induction on overgeneral autobiographical memory. Memory, 21(6), 751-761. doi:10.1080/09658211.2012.756039

Sumner, J. A. (2012). The mechanisms underlying overgeneral autobiographical memory: An evaluative review of evidence for the CaR-FA-X model. Clinical Psychology Review, 32(1), 34-48. doi:10.1016/j.cpr.2011.10.003

Sumner, J. A., Griffith, J. W., \& Mineka, S. (2010). Overgeneral autobiographical memory as a predictor of the course of depression: A meta-analysis. Behaviour Research and Therapy, 48(7), 614-625. doi:10.1016/j.brat.2010.03.013

Sumner, J. A., Griffith, J. W., \& Mineka, S. (2011). Examining the mechanisms of overgeneral autobiographical memory: Capture and rumination, and impaired executive control. Memory, 19(2), 169-183. doi:10.1080/09658211.2010.541467

Sumner, J. A., Mineka, S., Adam, E. K., Craske, M. G., Vrshek-Schallhorn, S., WolitzkyTaylor, K., \& Zinbarg, R. E. (2014). Testing the CaR-FA-X Model: Investigating the mechanisms underlying reduced autobiographical memory specificity in individuals with and without a history of depression. Journal of Abnormal Psychology, 123(3), 471486. doi:10.1037/a0037271

Sumner, J. A., Mineka, S., \& McAdams, D. P. (2012). Specificity in autobiographical memory narratives correlates with performance on the Autobiographical Memory Test and prospectively predicts depressive symptoms. Memory, 21(6), 646-656. doi:10.1080/09658211.2012.746372

Taylor, J. A. (1953). A personality scale of manifest anxiety. Journal of Abnormal Psychology, 48(2), 285-290. doi:10.1037/h0056264

Trew, J. L. (2011). Exploring the roles of approach and avoidance in depression: An integrative model. Clinical Psychology Review, 31(7), 1156-1168. doi:10.1016/j.cpr.2011.07.007 
Treynor, W., Gonzalez, R., \& Nolen-Hoeksema, S. (2003). Rumination reconsidered: A psychometric analysis. Cognitive Therapy and Research, 27(3), 247-259. doi:10.1023/A:1023910315561

Uzer, T. (2016). Retrieving autobiographical memories: How different retrieval strategies associated with different cues explain reaction time differences. Acta psychologica, 164, 144-150. doi:10.1016/j.actpsy.2016.01.004

van Vreeswijk, M. F., \& de Wilde, E. J. (2004). Autobiographical memory specificity, psychopathology, depressed mood and the use of the Autobiographical Memory Test: A meta-analysis. Psychological Bulletin, 42(6), 731-743. doi:10.1016/S00057967(03)00194-3

von Hippel, P. T. (2015). The heterogeneity statistic $I^{2}$ can be biased in small meta-analyses. BMC Medical Research Methodology, 15(1), 2-8. doi:10.1186/s12874-015-0024-Z

Watkins, E. R., Baeyens, C. B., \& Read, R. (2009). Concreteness training reduces dysphoria: Proof-of-principle for repeated cognitive bias modification in depression. Journal of Abnormal Psychology, 118(1), 55-64. doi:10.1037/a0013642

Watkins, E., Taylor, R., Byng, R., Baeyens, C., Read, R., Pearson, K., \& Watson, L. (2012). Guided self-help concreteness training as an intervention for major depression in primary care: A Phase II randomized controlled trial. Psychological Medicine, 42(7), 1359-1371. doi:10.1017/S0033291711002480

Watkins, E. R. (2016). Rumination-focused cognitive-behavioral therapy for depression. New York, NY: The Guilford Press.

Wegner, D. M., \& Zanakos, S. (1994). Chronic thought suppression. Journal of Personality, 62(4), 616-640. doi:DOI:10.1111/j.1467-6494.1994.tb00311.x

Wenzlaff, R. M., \& Wegner, D. M. (2000). Thought suppression. Annual Review of Psychology, 51, 59-91. doi:10.1146/annurev.psych.51.1.59

Williams, J. M. G. (1996). Depression and the specificity of autobiographical memory. In D. C. Rubin (Ed.), Remembering our past: Studies in autobiographical memory (pp. 244 267). Cambridge, England: Cambridge University Press.

Williams, J. M. G., Barnhofer, T., Crane, C., Herman, D., Raes, F., Watkins, E., \& Dalgleish, T. (2007). Autobiographical memory specificity and emotional disorder. Psychological Bulletin, 133(1), 122-148. doi:10.1037/0033-2909.133.1.122

Williams, J. M. G., \& Broadbent, K. (1986). Autobiographical memory in suicide attempters. Journal of Abnormal Psychology, 95(2), 144-149. doi:http://dx.doi.org/10.1037/0021- 
843X.95.2.144

Williams, J. M. G., Healy, H. G., \& Ellis, N. C. (1999). The effect of imageability and predicability of cues in autobiographical memory. The Quarterly Journal of Experimental Psychology, 52A(3), 555-579.

Williams, J. M. G., Stiles, W. B., \& Shapiro, D. A. (1999). Cognitive mechanisms in the avoidance of painful and dangerous thoughts: Elaborating the assimilation model. Cognitive Therapy and Research, 23(3), 285-306. doi:10.1023/A:1018743615228 


\section{Appendix A: AMT and AMT-R Cue-sets}

Cue-sets were taken from Sumner, Mineka, and McAdams (2012).

$\begin{array}{ll}\text { Cue-set } 1 & \text { Cue-set } 2 \\ \text { peaceful } & \text { energetic } \\ \text { failure } & \text { clumsy } \\ \text { safe } & \text { ambitious } \\ \text { hurt } & \text { sad } \\ \text { proud } & \text { surprised } \\ \text { frustrated } & \text { tense } \\ \text { successful } & \text { calm } \\ \text { regret } & \text { disappoint } \\ \text { brave } & \text { hopeful } \\ \text { inferior } & \text { lonely }\end{array}$

Cue-set 1 practice items

tree, book

Cue-set 2 practice items

window, hand 


\section{Appendix B: AMT Instructions}

\section{Instructions for Specific Memory Task}

In a moment, I'm going to give you a booklet where you can write memories. Each page of this booklet has one word. For each word, I want you to think of a personally experienced memory that the word reminds you of, and write a description of it in the space provided. also want you to rate how pleasant the memory is between one and five. One means extremely unpleasant. Five means extremely pleasant.

This study is completely anonymous, so your responses won't be linked to you in any way.

This is an example of what each page of the booklet looks like

boats

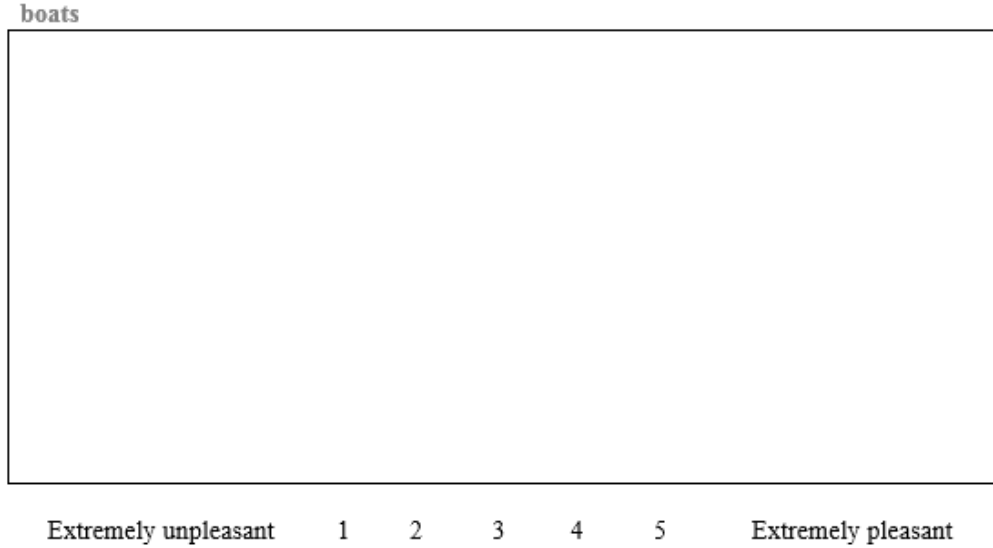

In the example above, the word is "boats", and there is a box where you would write your memory. You would rate how pleasant the memory is at the bottom by circling the appropriate number.

For each word I want you to remember a specific event that the word reminds you of. A specific event is something that:

- happened on one particular occasion,

- happened at a particular time and place,

- lasted less than one day,

- and happened at least one week ago.

For example, if the word was "boats" it would be okay to write, "Two weeks ago, I ate lunch with my friend at the waterfront." It is a specific event because it happened on one particular occasion, at a particular time and place, it lasted less than one day, and it happened at least one week ago.

But it would not be okay to write, "I always enjoy eating lunch with my friend at the waterfront" because that does not refer to a specific event

When you rate the memory, think back to when the event happened, and decide how pleasant or unpleasant it was.

Remember, I want you to think of a specific event that each word reminds you of. A specific event is something that:

- happened on one particular occasion,

- happened at a particular time and place,

- lasted less than one day,

- ahd happened at least one week ago.

Please do not use the same event more than once. If you can't think of a memory, just leave the page blank. You will have one minute to think of and write down each memory and rate how pleasant it was.

If you have any questions about this, you can ask me now. 


\section{Appendix C: AMT-R instructions}

Instructions for Category Memory Task

In a moment, I'm going to give you a booklet where you can write memories. Each page of this booklet has one word. For each word, I want you to think of a personally experienced memory that the word reminds you of, and write a description of it in the space provided. I also want you to rate how pleasant the memory is between one and five. One means extremely unpleasant. Five means extremely pleasant.

This study is completely anonymous, so your responses won't be linked to you in any way.

This is an example of what each page of the booklet looks like:

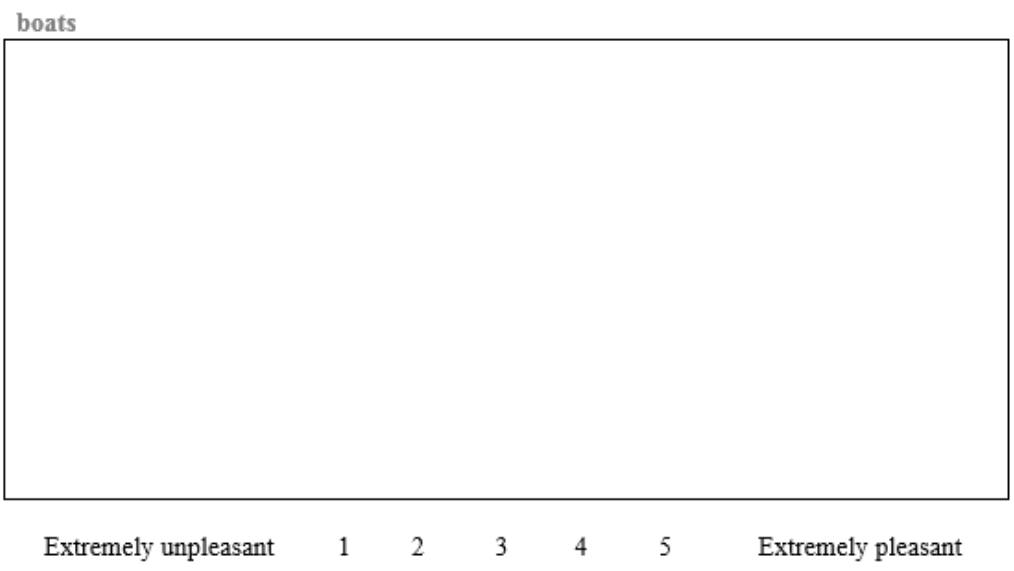

In the example above, the word is "boats", and there is a box where you would write your memory. You would rate how pleasant the memory is at the bottom by circling the appropriate number.

For each word, I want you to remember a certain category of event that the word reminds you of. In other words, a series of similar events that happened to you at different times.

For example, if the word was "boats," it would be okay to write, "I always enjoy eating lunch with my friend at the waterfront." It refers to a series of similar events, the times you ate lunch with your friend at the waterfront.

But it would not be okay to write, "Two weeks ago, I ate lunch with my friend at the waterfront," because that does not refer to a category of events.

When you rate the memory, think back to when the events happened, and decide how pleasant or unpleasant this type of event is for you.

Remember, I want you to think of a certain category of event that each word reminds you of In other words, a series of similar events that happened to you at different times.

Please do not use the same category of event more than once. If you can't think of a memory, just leave the page blank. You will have one minute to think of and write down each memory and rate how pleasant it was.

If you have any questions about this, you can ask me now. 


\section{Appendix D: AMT and AMT-R Practice Trial Instructions}

Instructions pertaining to pleasantness rating were omitted in the no-pleasantness rating condition in Study 4.

Don't turn the page until I tell you to.

We'll practice twice.

You can see the word. Now write your memory. Also rate the memory. I'll tell you to stop in one minute.

[Researcher waits one minute, then says:]

... Stop. Turn the page. You can see the word. Now write your memory. Also rate the memory.

[Researcher waits one minute, then says:]

... Stop. Does anyone have any final questions about what they are supposed to do?

[Researcher answers any questions from participants, then says:]

From now on I won't give you any more instructions. I'll just tell you to stop and turn the page after a minute for each word. We'll start now. Turn the page and begin. 


\section{Appendix E: Coding Scheme for AMT and AMT-R}

\section{General guidelines}

Responses should be coded as: Specific, Extended, Categoric, Semantic Associate, Error, or Omission.

Use your own discretion and apply your own knowledge of the world when coding responses. Is the event likely to have occurred in a day or less, or longer? Is the response referring to a single, unique event or is it referring to a category of events?

Responses for which there are specific aspects as well as other aspects (e.g., categoric) should be given a specific code.

If two responses are given, code the first response.

\section{Specific}

A specific response refers to a unique, personally experienced event (i.e., one that happened at a particular time and place) and that lasted no more than a day. The time and place do not need to be mentioned.

\section{Categoric}

A categoric response refers to a category of personally experienced events, that is, that have happened at least twice. These could refer to repeated events or re-occurrences in some way. E.g., "Before I came to university I would meditate in my room."

\section{Extended}

An extended response refers to a personally experienced event that lasted longer than one day. An extended response could refer to a specified period of time longer than a day, e.g., "The first week back from the holidays I wanted to go back home, not live in the hostel.", or circumscribed in other ways, e.g., "I was sick from year 8 to 9 and couldn't leave the house to go to school."

\section{Semantic Associate}

A semantic associate response refers to semantic information that does not contain overt references to episodes. That is, it does not contain information about a personally experienced event. A single noun qualifies as a semantic associate, e.g., "beach". Events that did not happen are coded as semantic associates because they cannot be remembered episodes, e.g., "Not talking to/asking out my first crush at intermediate." Semantic associate should be the code if the information doesn't fit into the error code (described below) and the information is more general knowledge rather than referring to an episode.

\section{Error}

Error responses are future-oriented statements, repetitions of previous memories, or incomprehensible statements.

\section{Errors on the AMT}

The AMT (but not the AMT-R) included an instruction that the memory should be at least one week old. Therefore, give the response an error code if the memory is specific, but less than one week old. Benefit of the doubt should be given (i.e., not coded as an error) unless the time mentioned is definitely less than one week old. To aid in coding, the day of the week the session occurred on is recorded with each corresponding response. Here are some rulesof-thumb: 
- "The other day" is a turn of phrase that very likely refers to an event recent enough that it is less than one week ago. Therefore responses with this turn of phrase should be coded as an error.

- "This week" indicates the event happened within the past week. E.g., "[] has been talking a lot this week about his plans after university". This should be coded as an error.

- If a day of the week is mentioned (e.g., "on Monday"), the event likely refers to an event that happened within the last week, e.g., "I got a C+ on my stats project assignment on Monday. I thought I was going to fail". This should be coded as an error.

If the response uses the word "last", e.g., "last Wednesday", refer to the day of the week the response was made to make a judgement on whether the response was made within the last week or not. For example, consider the response: "Last Wednesday I went to the movies." If the response was made on a Tuesday, the participant is likely referring to within the last seven days. However, if the response was made on Thursday, they are likely not referring to yesterday. Rather, they are referring to Wednesday in the previous week-i.e., longer than one week ago.

- References to "earlier in the week" are an error, e.g., "Earlier on in the week my neighbour had a fight with my friend." This is an error because it definitely happened less than one week ago.

\section{Omission}

Omissions are coded when there is no response to the cue. This is most often a blank page. Overt statements about not being able to think of a response should be coded as an omission, e.g., "Sorry. Can't think of one". 


\section{Appendix F: Self-Report Measures}

\section{Acceptance and Action Questionnaire II (AAQ-II; Bond et al., 2011)}

Below you will find a list of statements. Please rate how true each statement is for you by circling a number next to it. Use the scale below to make your choice.

\begin{tabular}{|c|c|c|c|c|c|c|}
\hline 1 & 2 & 3 & 4 & 5 & 6 & 7 \\
\hline $\begin{array}{c}\text { never } \\
\text { true }\end{array}$ & $\begin{array}{c}\text { very seldom } \\
\text { true }\end{array}$ & $\begin{array}{c}\text { seldom } \\
\text { true }\end{array}$ & $\begin{array}{c}\text { sometimes } \\
\text { true }\end{array}$ & $\begin{array}{c}\text { frequently } \\
\text { true }\end{array}$ & $\begin{array}{c}\text { almost } \\
\text { always true }\end{array}$ & $\begin{array}{c}\text { always } \\
\text { true }\end{array}$ \\
\hline
\end{tabular}

\begin{tabular}{|c|c|c|c|c|c|c|c|}
\hline $\begin{array}{l}\text { 1. My painful experiences and memories make it difficult } \\
\text { for me to live a life that I would value. }\end{array}$ & 1 & 2 & 3 & 4 & 5 & 6 & 7 \\
\hline 2. I'm afraid of my feelings. & 1 & 2 & 3 & 4 & 5 & 6 & 7 \\
\hline $\begin{array}{l}\text { 3. I worry about not being able to control my worries and } \\
\text { feelings. }\end{array}$ & 1 & 2 & 3 & 4 & 5 & 6 & 7 \\
\hline $\begin{array}{l}\text { 4. My painful memories prevent me from having a } \\
\text { fulfilling life. }\end{array}$ & 1 & 2 & 3 & 4 & 5 & 6 & 7 \\
\hline 5. Emotions cause problems in my life. & 1 & 2 & 3 & 4 & 5 & 6 & 7 \\
\hline $\begin{array}{l}\text { 6. It seems like most people are handling their lives better } \\
\text { than I am. }\end{array}$ & 1 & 2 & 3 & 4 & 5 & 6 & 7 \\
\hline 7. Worries get in the way of my success. & 1 & 2 & 3 & 4 & 5 & 6 & 7 \\
\hline
\end{tabular}




\section{Cognitive Behavioral Avoidance Scale (CBAS; Ottenbreit \& Dobson, 2004)}

Instructions: Different people use different strategies to deal with situations and problems in their lives. Below are a number of strategies that people may use to deal with situations and problems. A number of the items below refer to dealing with situations at work or university. If you are not currently working or attending university, answer these items instead using your daily duties and activities. Please read each statement carefully and indicate how true, in general, each statement is for you using the following key:

$1=$ Not at all true for me $2=$ Somewhat true for me $3=$ Moderately true for me $4=$ Very much true for me $5=$ Extremely true for me

\begin{tabular}{|c|c|c|c|c|c|}
\hline 1. I avoid attending social activities. & 1 & 2 & 3 & 4 & 5 \\
\hline $\begin{array}{l}\text { 2. When uncertain about my future, I do not sit down and think about what I } \\
\text { really want. }\end{array}$ & 1 & 2 & 3 & 4 & 5 \\
\hline $\begin{array}{l}\text { 3. I would like to achieve things at work/university, but I have to accept my } \\
\text { limits. }\end{array}$ & 1 & 2 & 3 & 4 & 5 \\
\hline $\begin{array}{l}\text { 4. I do not do what is needed to follow through with achievement goals I } \\
\text { have set for myself. }\end{array}$ & 1 & 2 & 3 & 4 & 5 \\
\hline $\begin{array}{l}\text { 5. In order to avoid feelings of disappointment, I just try not to get too } \\
\text { serious about work/university. }\end{array}$ & 1 & 2 & 3 & 4 & 5 \\
\hline 6. Rather than try new activities, I tend to stick with the things I know. & 1 & 2 & 3 & 4 & 5 \\
\hline 7. I choose to turn down opportunities to further my education/career. & 1 & 2 & 3 & 4 & 5 \\
\hline $\begin{array}{l}\text { 8. I do not answer the phone in case people are calling with social } \\
\text { invitations. }\end{array}$ & 1 & 2 & 3 & 4 & 5 \\
\hline 9. I quit activities that challenge me too much. & 1 & 2 & 3 & 4 & 5 \\
\hline 10. I try not to think about problems in my personal relationships. & 1 & 2 & 3 & 4 & 5 \\
\hline $\begin{array}{l}\text { 11. I think to myself that I will not be able to complete really challenging } \\
\text { tasks. }\end{array}$ & 1 & 2 & 3 & 4 & 5 \\
\hline $\begin{array}{l}\text { 12. While I know I should make decisions about my personal relationships, I } \\
\text { just let things go on as they are. }\end{array}$ & 1 & 2 & 3 & 4 & 5 \\
\hline 13. I avoid trying new activities that hold the potential for failure. & 1 & 2 & 3 & 4 & 5 \\
\hline $\begin{array}{l}\text { 14. I do not go out to events when I know there will be a lot of people I do } \\
\text { not know. }\end{array}$ & 1 & 2 & 3 & 4 & 5 \\
\hline $\begin{array}{l}\text { 15. Instead of thinking about problems in my social life, I tell myself that I } \\
\text { prefer to be alone. }\end{array}$ & 1 & 2 & 3 & 4 & 5 \\
\hline 16. I do not discuss/address tension that builds in a friendship. & 1 & 2 & 3 & 4 & 5 \\
\hline 17. I find that I often want to leave social gatherings. & 1 & 2 & 3 & 4 & 5 \\
\hline $\begin{array}{l}\text { 18. I do not try to think about ways to improve my work/university } \\
\text { performance. }\end{array}$ & 1 & 2 & 3 & 4 & 5 \\
\hline
\end{tabular}




\begin{tabular}{|c|c|c|c|c|c|}
\hline 19. I try not to think about my future and what I will do with my life. & 1 & 2 & 3 & 4 & 5 \\
\hline 20. I just wait out tension in my relationships hoping that it will go away. & 1 & 2 & 3 & 4 & 5 \\
\hline 21. I tend to make up excuses to get out of social activities. & 1 & 2 & 3 & 4 & 5 \\
\hline 22. There is nothing I can do to improve problems in my relationships. & 1 & 2 & 3 & 4 & 5 \\
\hline 23. I turn down opportunities to socialize with the opposite sex. & 1 & 2 & 3 & 4 & 5 \\
\hline 24. I tend to remain to myself during social gatherings or activities. & 1 & 2 & 3 & 4 & 5 \\
\hline 25. I avoid making decisions about my future. & 1 & 2 & 3 & 4 & 5 \\
\hline $\begin{array}{l}\text { 26. When I experience confusion in my relationships, I do not try to figure } \\
\text { things out. }\end{array}$ & 1 & 2 & 3 & 4 & 5 \\
\hline $\begin{array}{l}\text { 27. While I know that I have to make some important decisions about } \\
\text { university/work, I just do not get down to it. }\end{array}$ & 1 & 2 & 3 & 4 & 5 \\
\hline 28. Rather than getting out and doing things, I just sit at home. & 1 & 2 & 3 & 4 & 5 \\
\hline $\begin{array}{l}\text { 29. I distract myself when I start to think about my work/university } \\
\text { performance. }\end{array}$ & 1 & 2 & 3 & 4 & 5 \\
\hline $\begin{array}{l}\text { 30. I do not bother thinking about how to solve problems in my family - it is } \\
\text { useless. }\end{array}$ & 1 & 2 & 3 & 4 & 5 \\
\hline 31. I find myself avoiding tasks and assignments that are really important. & 1 & 2 & 3 & 4 & 5 \\
\hline
\end{tabular}




\section{Depression Anxiety Stress Scales (DASS; Lovibond \& Lovibond, 1995)}

Please read each statement and circle a number $0,1,2$ or 3 which indicates how much the statement applied to you over the past week. There are no right or wrong answers. Do not spend too much time on any statement.

The rating scale is as follows:

0 Did not apply to me at all

1 Applied to me to some degree, or some of the time

2 Applied to me to a considerable degree, or a good part of time

3 Applied to me very much, or most of the time

\begin{tabular}{|l|l|llll|}
\hline 1 & I found it hard to wind down & 0 & 1 & 2 & 3 \\
\hline 2 & I was aware of dryness of my mouth & 0 & 1 & 2 & 3 \\
\hline 3 & I couldn't seem to experience any positive feeling at all & 0 & 1 & 2 & 3 \\
\hline 4 & $\begin{array}{l}\text { I experienced breathing difficulty (eg, excessively rapid breathing, } \\
\text { breathlessness in the absence of physical exertion) }\end{array}$ & 0 & 1 & 2 & 3 \\
\hline 5 & I found it difficult to work up the initiative to do things & & & & \\
\hline 6 & I tended to over-react to situations & 0 & 1 & 2 & 3 \\
\hline 7 & I experienced trembling (eg, in the hands) & 0 & 1 & 2 & 3 \\
\hline 8 & I felt that I was using a lot of nervous energy & 0 & 1 & 2 & 3 \\
\hline 9 & I was worried about situations in which I might panic and make & 0 & 1 & 2 & 3 \\
\hline 10 & a fool of myself & 0 & 1 & 2 & 3 \\
\hline 11 & I felt that I had nothing to look forward to & & & & \\
\hline 12 & I found it difficult to relax & 0 & 1 & 2 & 3 \\
\hline 13 & I felt down-hearted and blue & 0 & 1 & 2 & 3 \\
\hline 14 & I was intolerant of anything that kept me from getting on with & 0 & 1 & 2 & 3 \\
\hline 15 & what I was doing & 0 & 1 & 2 & 3 \\
\hline 16 & I felt I was close to panic & 0 & 1 & 2 & 3 \\
\hline 17 & I felt I wasn't worth much as a person & & & & \\
\hline 18 & I felt that I was rather touchy & 0 & 1 & 2 & 3 \\
\hline 19 & I was aware of the action of my heart in the absence of physical \\
\hline 20 & I felt scared without any good reason & 0 & 1 & 2 & 3 \\
\hline 21 & I felt that life was meaningless & 0 & 1 & 2 & 3 \\
\hline
\end{tabular}


White Bear Suppression Inventory (WBSI; Wegner \& Zanakos, 1994)

This survey is about thoughts. There are no right or wrong answers, so please respond honestly to each of the items below. Be sure to answer every item by circling the appropriate letter beside each.

\begin{tabular}{|c|c|c|c|c|}
\hline A & B & C & D & E \\
\hline $\begin{array}{c}\text { Strongly } \\
\text { Disagree }\end{array}$ & Disagree & $\begin{array}{c}\text { Neutral or } \\
\text { Don't Know }\end{array}$ & Agree & Strongly Agree \\
\hline
\end{tabular}

\begin{tabular}{|l|c|c|c|c|c|}
\hline 1. There are things I prefer not to think about. & A & B & C & D & E \\
\hline 2. Sometimes I wonder why I have the thoughts I do. & A & B & C & D & E \\
\hline 3. I have thoughts that I cannot stop. & A & B & C & D & E \\
\hline 4. There are images that come to mind that I cannot erase. & A & B & C & D & E \\
\hline 5. My thoughts frequently return to one idea. & A & B & C & D & E \\
\hline 6. I wish I could stop thinking of certain things. & A & B & C & D & E \\
\hline 7. Sometimes my mind races so fast I wish I could stop it. & A & B & C & D & E \\
\hline 8. I always try to put problems out of mind. & A & B & C & D & E \\
\hline 9. There are thoughts that keep jumping into my head. & A & B & C & D & E \\
\hline 10. There are things that I try not to think about. & A & B & C & D & E \\
\hline 11. Sometimes I really wish I could stop thinking. & A & B & C & D & E \\
\hline 12. I often do things to distract myself from my thoughts. & A & B & C & D & E \\
\hline 13. I have thoughts that I try to avoid. & A & B & C & D & E \\
\hline 14. There are many thoughts that I have that I don't tell anyone. & A & B & C & D & E \\
\hline $\begin{array}{l}\text { 15. Sometimes I stay busy just to keep thoughts from intruding on my } \\
\text { mind. }\end{array}$ & A & B & C & D & E \\
\hline
\end{tabular}

